Supporting information for:

\title{
Strategic Construction of Ethene-Bridged BODIPY Arrays with Absorption Bands Reaching Near-Infrared II Region
}

Qinghua Wu, Zhengxin Kang, Qingbao Gong, Xing Guo, Hua Wang, Dandan Wang, Lijuan Jiao, * Erhong Hao*

Laboratory of Functionalized Molecular Solids, Ministry of Education, College of Chemistry and Materials Science, Anhui Normal University, Wuhu, 241002, China.

*To whom correspondence: jiao421@ahnu.edu.cn; haoehong@ahnu.edu.cn.

\section{Contents}

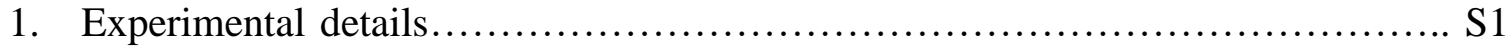

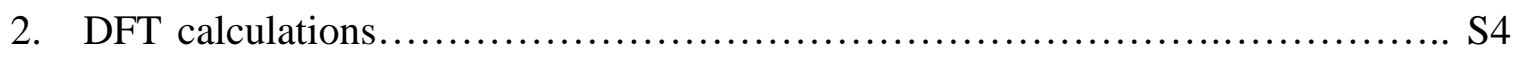

3. Synthesis and characterization............................................... $\mathrm{S} 12$

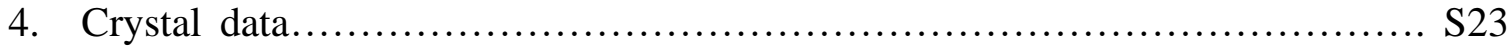

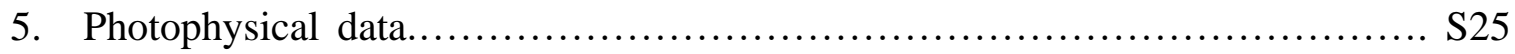

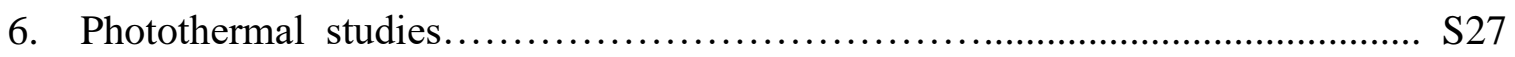

7. Characterization and properties of nanoparticles................................ 29

8. $\quad{ }^{1} \mathrm{H}$ NMR, ${ }^{13} \mathrm{C}$ NMR and HRMS for all new compounds........................................ 331

9. Optimized Geometries of the Compounds.......................................68 


\section{Experimental details}

General information. Reagents and solvents were used as received from commercial suppliers (Energy Chemicals, Shanghai, China) unless noted otherwise. All reactions were performed in oven-dried or flame-dried glassware unless stated otherwise and were monitored by TLC using $0.25 \mathrm{~mm}$ silica gel plates with UV indicator (60F-254). ${ }^{1} \mathrm{H}$ and ${ }^{13} \mathrm{C}$ NMR spectra were recorded on a 400 or $500 \mathrm{MHz}$ NMR spectrometer at room temperature. Chemical shifts $(\delta)$ are given in ppm relative to internal TMS. High-resolution mass spectra (HRMS) were obtained using MALDI-TOF in positive mode.

Theoretical calculations. The ground state geometry was optimized by using DFT method at B3LYP/6-31G(d) level. The same method was used for vibrational analysis to verify that the optimized structures correspond to local minima on the energy surface. TD-DFT computations were used the optimized ground state geometries under the B3LYP/6-31G (d, p) theoretical level. The calculated molecules in dichloromethane were done using the Self-Consistent Reaction Field (SCRF) method and Polarizable Continuum Model (PCM). All the calculations were carried out by the methods implemented in Gaussian 09 package. ${ }^{1}$

Absorption and emission measurements. UV-visible absorption and fluorescence emission spectra were recorded on commercial spectrophotometers (Shimadzu UV-4100 and Edinburgh FS5 or FLS1000 spectrometers). All measurements were made at $25^{\circ} \mathrm{C}$, using $10 \mathrm{~mm}$ cuvettes. Relative fluorescence quantum yields of $\mathbf{1}$ was calculated using fluorescein ( $\phi=0.90$ in $0.1 \mathrm{M} \mathrm{NaOH}$ solution) as the standard, ${ }^{2 \mathrm{a}} 2$ was calculated using 1,7-diphenyl-3,5- di(p-methoxyphenyl)-azadipyrromethene ( $\phi=0.36$ in chloroform) as the reference, ${ }^{2 \mathrm{~b}} 3$ was calculated using ICG ( $\phi=0.12$ in DMSO) as the reference, ${ }^{2 \mathrm{c}}$ 4-6 were calculated using IR1061 ( $\phi=1.7 \%$ in DCM) as the reference. ${ }^{3}$ Dilute solutions $(0.01<\mathrm{A}<0.05)$ were used to minimize the reabsorption effects. Quantum yields were determined using the following equation: ${ }^{4}$ 


$$
\Phi_{x}=\Phi_{r} \times \frac{F_{x}}{F_{r}} \times \frac{1-10^{-A_{r}\left(\lambda_{\mathrm{ex}}\right)}}{1-10^{-A_{x}\left(\lambda_{\mathrm{ex}}\right)}} \times \frac{n_{x}^{2}}{n_{r}^{2}}
$$

where the subscripts $x$ and $r$ refer respectively to the BODIPY sample $x$ and reference (standard) fluorophore $r$ with known quantum yield $\Phi_{r}$ in a specific solvent; $F$ stands for the spectrally corrected, integrated fluorescence spectra; $A\left(\lambda_{\mathrm{ex}}\right)$ denotes the absorbance at the used excitation wavelength $\lambda_{\text {ex }}$ and $n$ represents the refractive index of the solvent (in principle at the average emission wavelength).

X-ray structure analysis. Crystals of compounds $\mathbf{2} \mathbf{C l}_{\mathbf{2}}$ suitable for X-ray analysis was obtained via the slow diffusion of petroleum ether into their dichloromethane solutions. The vial containing this solution was placed, loosely capped, to promote the crystallization. A suitable crystal was chosen and mounted on a glass fiber using grease. Data were collected using a diffractometer equipped with a graphite crystal monochromator situated in the incident beam for data collection at room temperature. Cell parameters were retrieved using SMART $^{5}$ software and refined using SAINT on all observed reflections. The determination of unit cell parameters and data collections were performed with Mo $K \alpha$ radiation $(\lambda)$ at $0.71073 \AA$ A. Data reduction was performed using the SAINT software, ${ }^{6}$ which corrects for Lp and decay. The structure was solved by the direct method using the SHELXS-97 ${ }^{7}$ program and refined by least squares method on $F^{2}$, SHELXL-2018/3, incorporated in SHELXTL V5.10. ${ }^{8}$ CCDC-1991866 (2Cl2) contain the supplementary crystallographic data for this paper. These data can be obtained free of charge from The Cambridge Crystallographic Data Centre via www.ccdc.cam.ac.uk/data_request/cif.

Photothermal experiments. To demonstrate the photothermal conversion behavior of 3-6, the solutions containing 3-6 at the concentrations of $50.0 \mu \mathrm{g} \mathrm{mL}^{-1}$ were irradiated for $300 \mathrm{~s}$ in $0.5 \mathrm{~mL}$ glass vials under $808 \mathrm{~nm}$ (arrays 3 and 4) or $980 \mathrm{~nm}$ (arrays 4-6) laser in toluene, respectively. Moreover, 3-NPs at the concentration of $50 \mu \mathrm{g} \mathrm{mL}^{-1}$ were irradiated by using a $808 \mathrm{~nm}$ laser at $0.1,0.25,0.5$ and $1 \mathrm{~W} \mathrm{~cm}^{-2}$ for $300 \mathrm{~s}, 3-\mathrm{NPs}$ at the 
concentration of $0-50 \mu \mathrm{g} \mathrm{mL} \mathrm{m}^{-1}$ were irradiated by using a $808 \mathrm{~nm}$ laser at $1 \mathrm{~W} \mathrm{~cm}^{-2}$ for 300 s. Photothermal conversion experiments of oligomers 3-6 and 3-NPs were conducted at a concentration of $50 \mu \mathrm{g} \mathrm{mL}^{-1}$ under $808 \mathrm{~nm}$ and $980 \mathrm{~nm}$ laser irradiation $\left(1 \mathrm{~W} \mathrm{~cm}^{-2}\right)$ for five minutes, and then cooled down to room temperature. Indocyanine green (ICG) as the reference was tested at a concentration of $50 \mu \mathrm{g} \mathrm{mL}^{-1}$ under $808 \mathrm{~nm}$ irradiation $(1 \mathrm{~W}$ $\mathrm{cm}^{-2}$ ) in water. The photothermal conversion efficiency $(\eta)$ values were calculated by a reported method. ${ }^{9}$

Self-assembly of 3NPs. 3NPs were fabricated by a reported method. ${ }^{10}$ Briefly, chloroform solution of $\mathbf{3}\left(200 \mu \mathrm{L}, 1 \mathrm{mg} \mathrm{mL}^{-1}\right)$ and chloroform solution of F127 (240 $\mu \mathrm{L}, 10 \mathrm{mg} \mathrm{mL}^{-1}$ ) were added to a flask. The obtained mixture was then dried under vacuum in a rotary evaporator to remove the chloroform completely. After that, DI water $(200 \mu \mathrm{L})$ was added, and the flask was placed under sonication for several minutes to give the aqueous dispersion of 3NPs. 


\section{DFT calculations}

various dimers
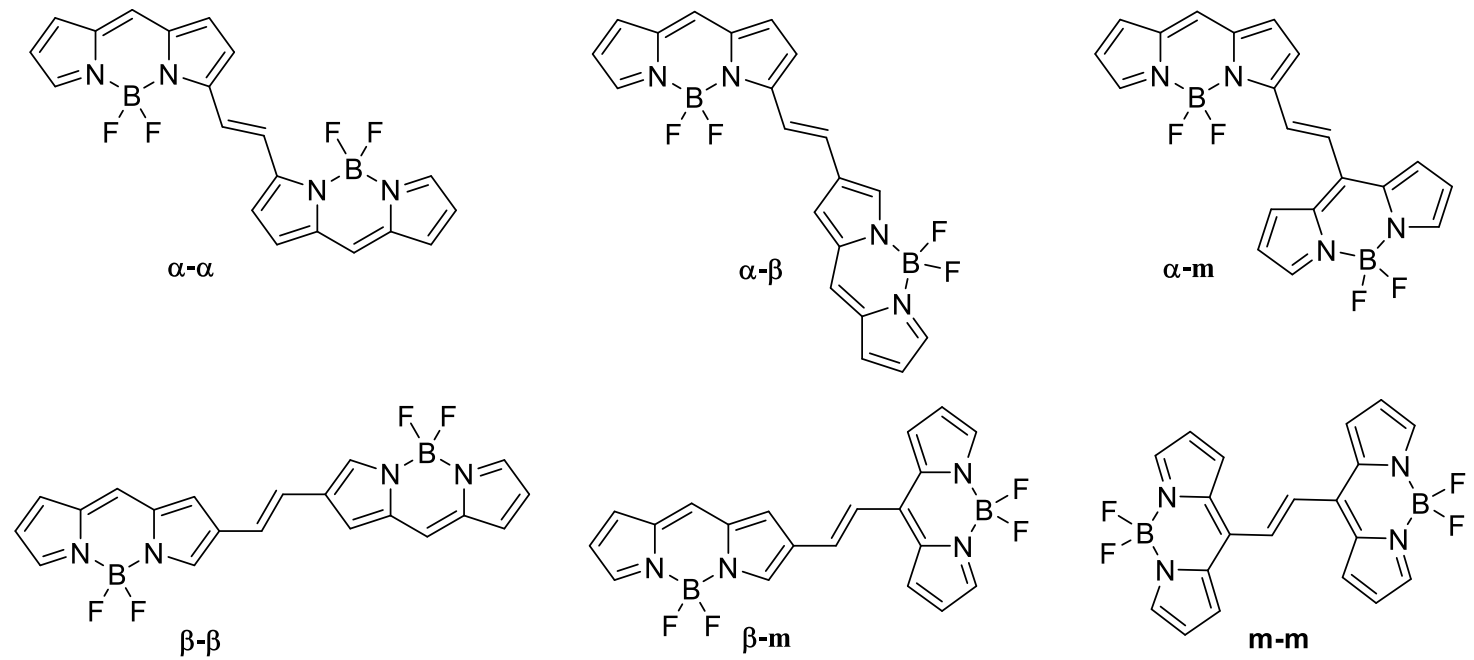

$\alpha-\alpha-\alpha(3)$

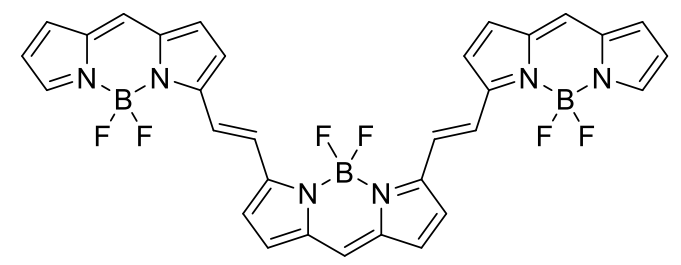

$\alpha-\alpha-\alpha-\alpha(4)$

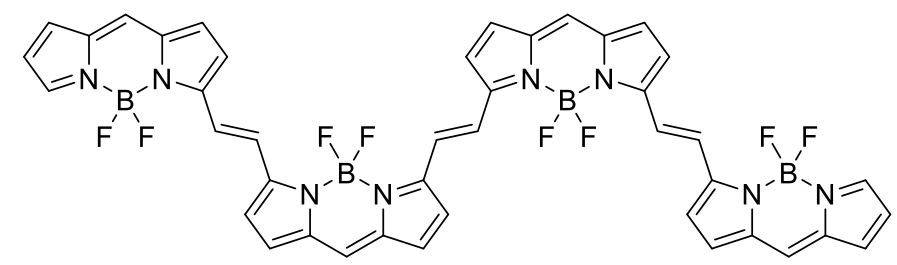

$\alpha-\alpha-\alpha-\alpha-\alpha(5)$

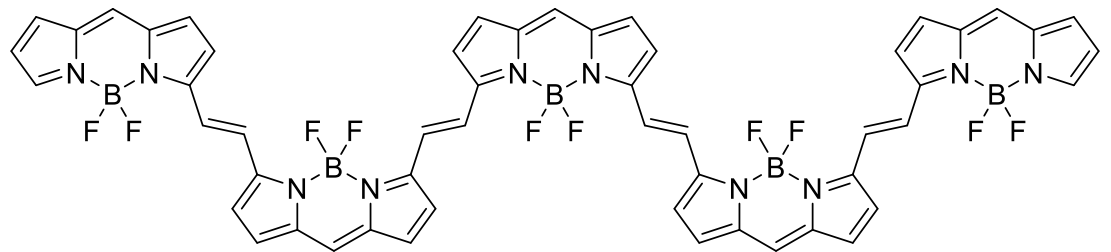

$\alpha-\alpha-\alpha-\alpha-\alpha-\alpha(6)$

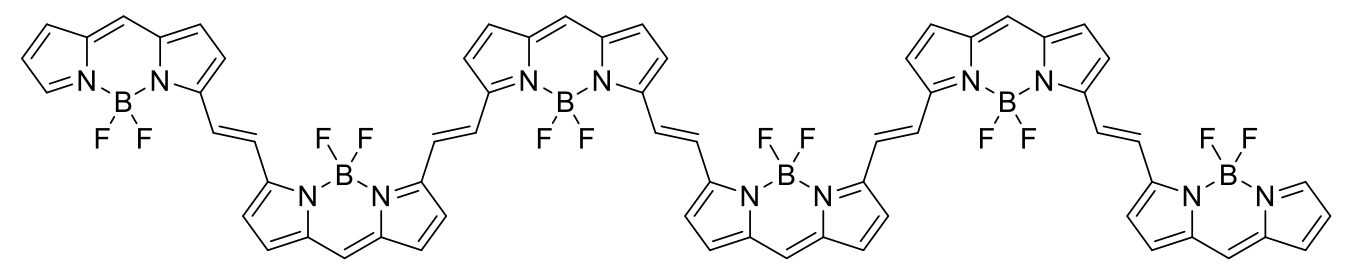

Scheme S1. Structure of possible ethene linked BODIPY dimesr and $\alpha-\alpha$ linked higher oligomers 3-6. Noted that meso-Ar groups were omitted. 


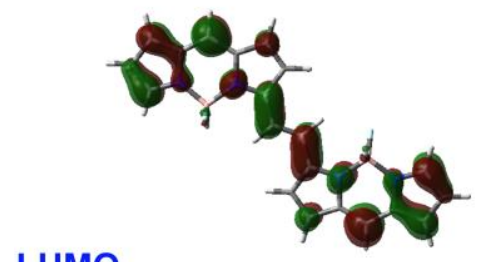

LUMO

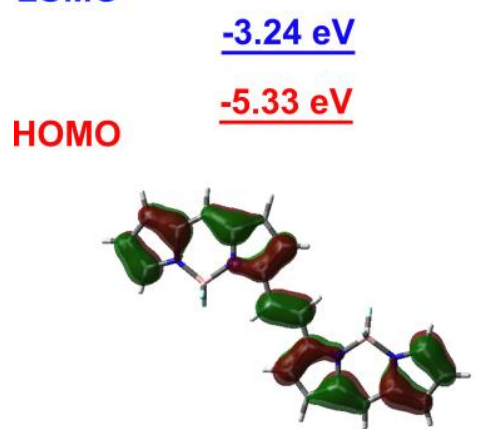

$\theta$ :

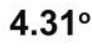

$\alpha-\alpha$

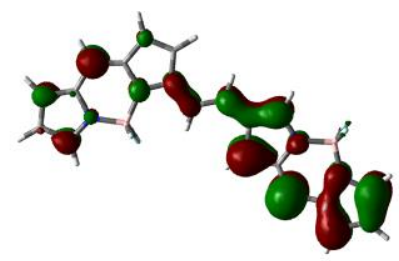

$-3.14 \mathrm{eV}$

$-5.34 \mathrm{eV}$

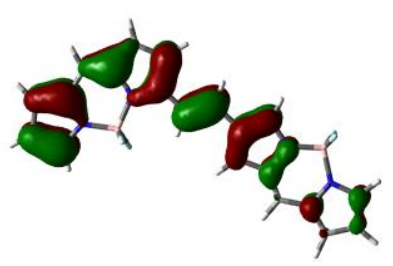

$2.98^{\circ}$ $\alpha-\beta$

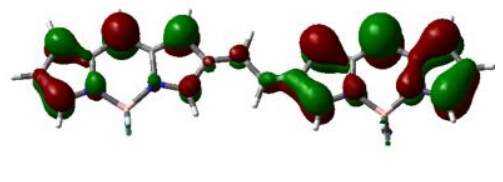

$\underline{-3.03 \mathrm{eV}}$

$-5.40 \mathrm{eV}$

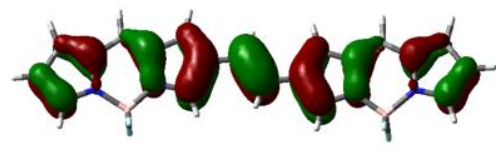

$4.51^{\circ}$

$\beta-\beta$

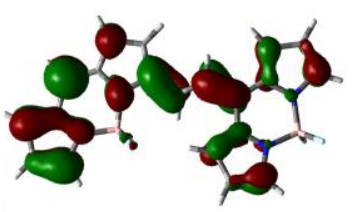

LUMO

$\underline{-3.45 \mathrm{eV}}$

HOMO

$\underline{-5.76 \mathrm{eV}}$

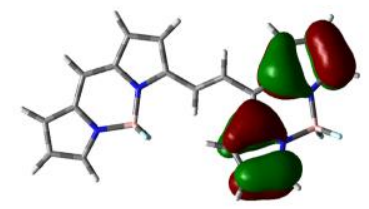

$\theta$ :

$15.21^{\circ}$
$\alpha-m$

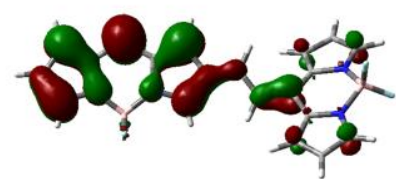

$\underline{-3.34 \mathrm{eV}}$

$-5.80 \mathrm{eV}$

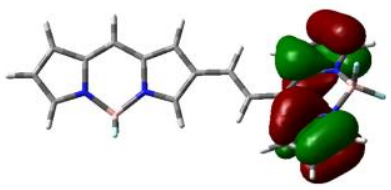

$39.04^{\circ}$

$\beta-m$

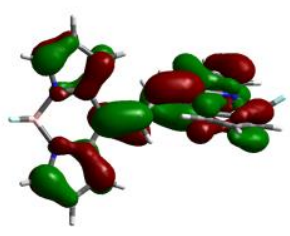

$-3.70 \mathrm{eV}$

Figure S1. Calculated HOMO/LUMO of energy-minimized calculated (Gaussian) ethene-linked BODIPY dimers. $\theta$ stands for the dihedral angels between adjacent dipyrrin planes. Calculation was performed at B3LYP/6-31G* level with Gaussian 09. 

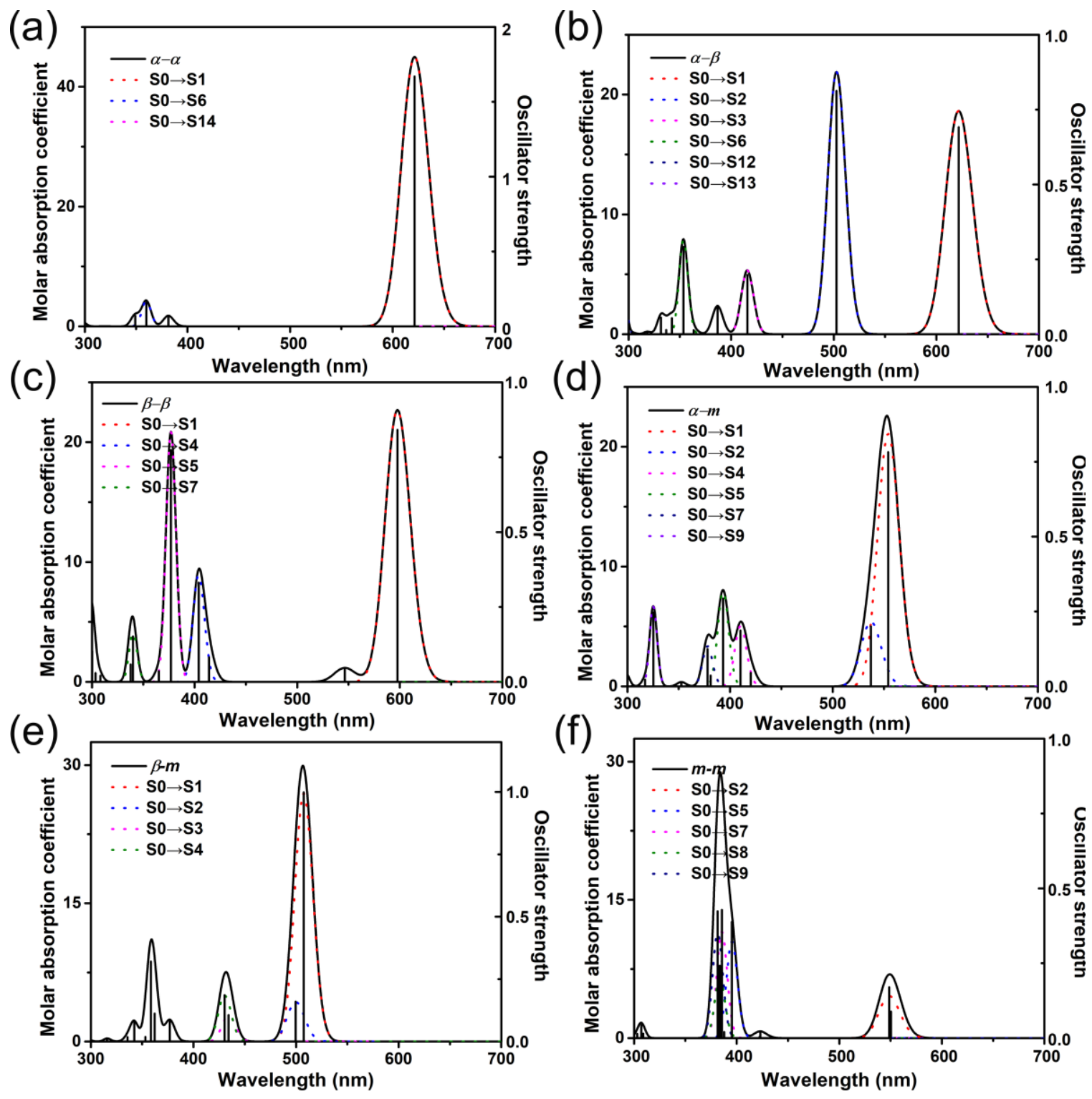

Figure S2. Calculated UV-vis absorption (a-f) of energy-minimized calculated (Gaussian) ethene linked BODIPY dimers. The unit for molar absorption coefficient is $10^{4} \mathrm{~L} / \mathrm{mol} / \mathrm{cm}$. Calculation was performed at B3LYP/6-31G* level with Gaussian 09. 


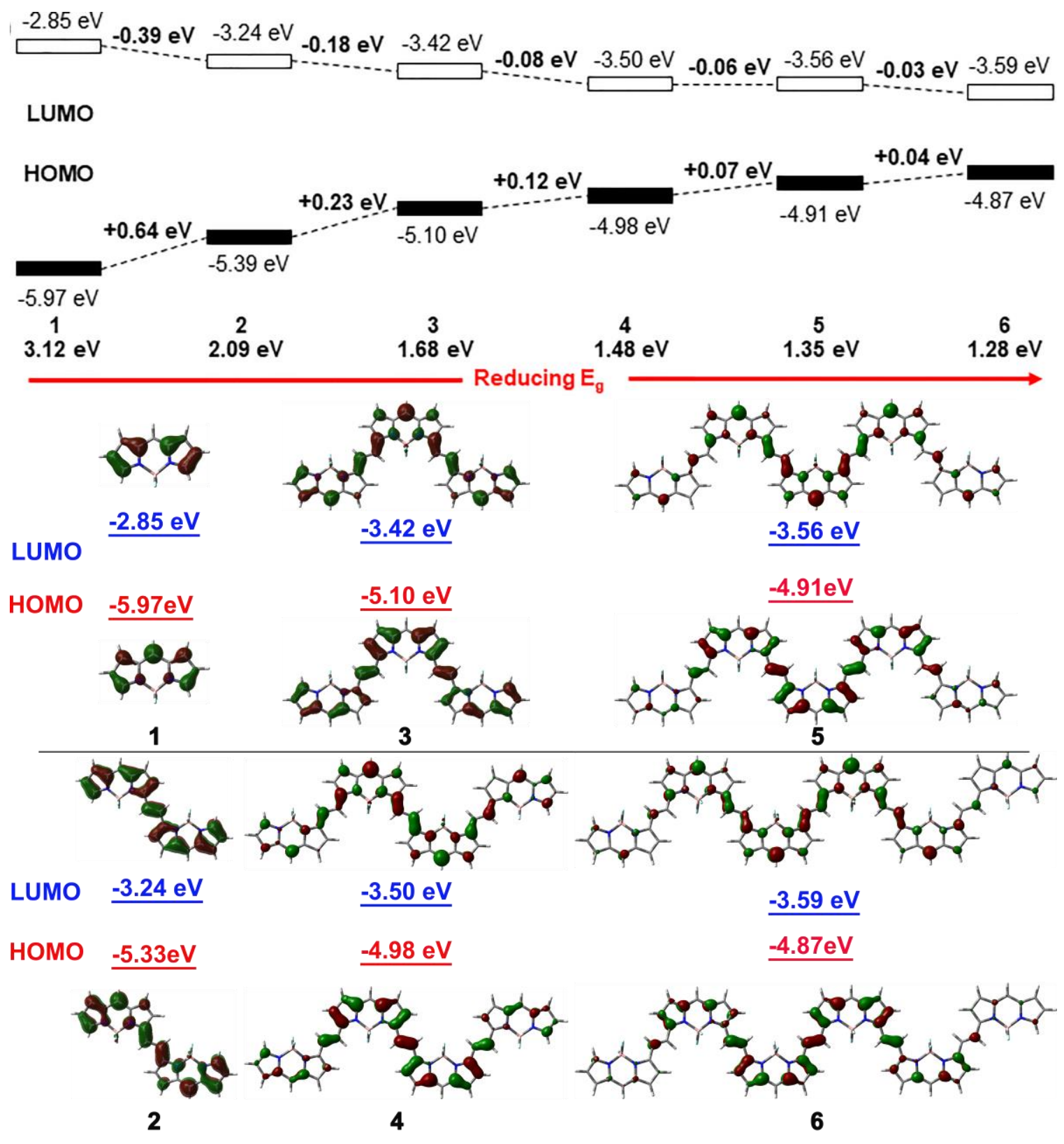

Figure S3. Calculated HOMO/LUMO of energy-minimized calculated (Gaussian) ethene linked BODIPYs 1-6. Calculation was performed at B3LYP/6-31G* level with Gaussian 09. 
(a)

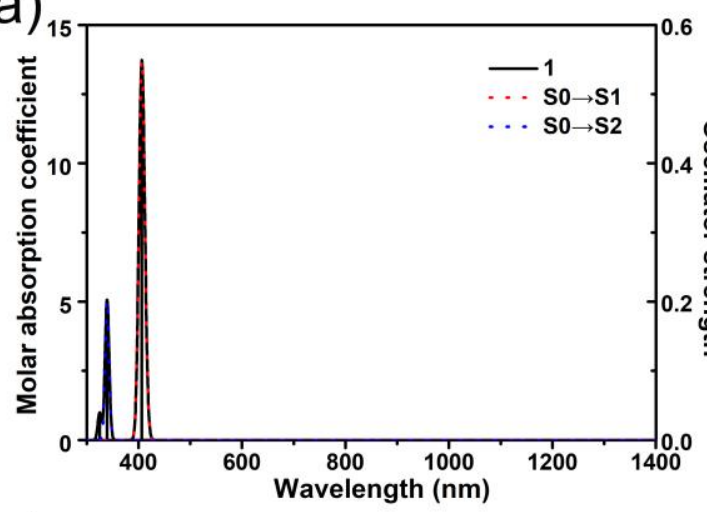

(c)

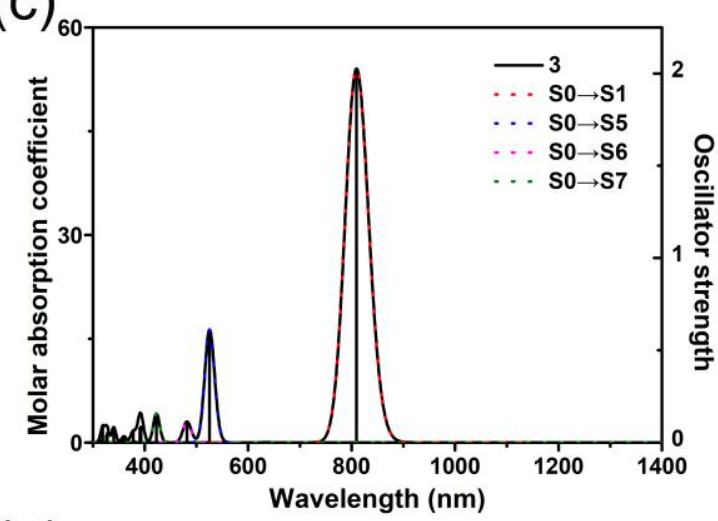

(e)

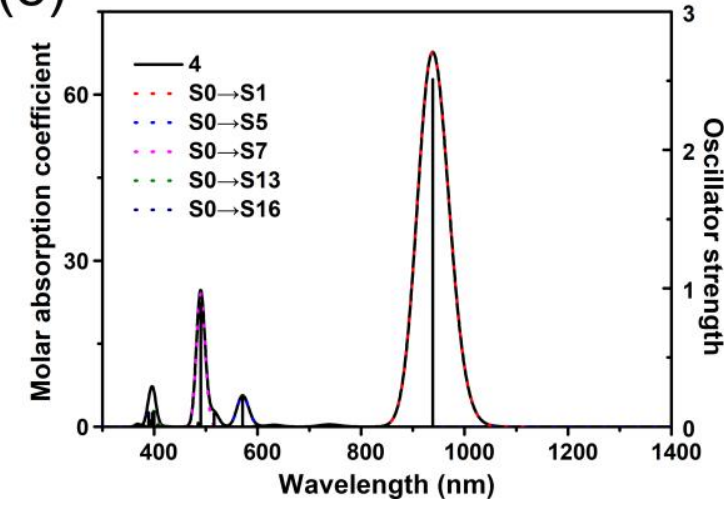

(b)

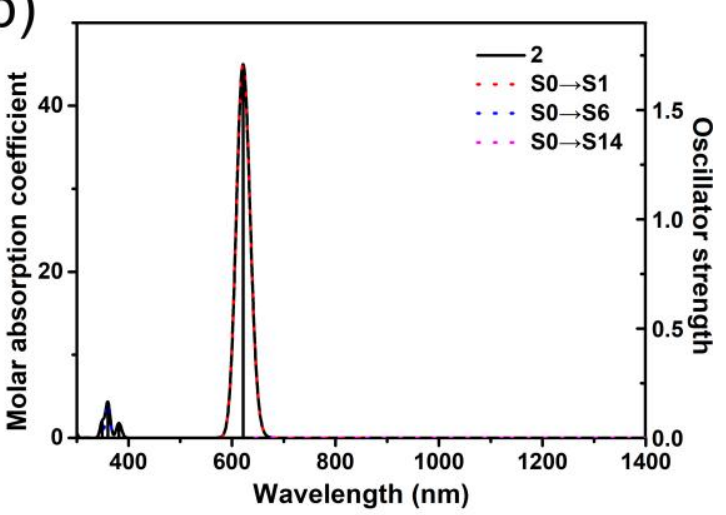

(d)

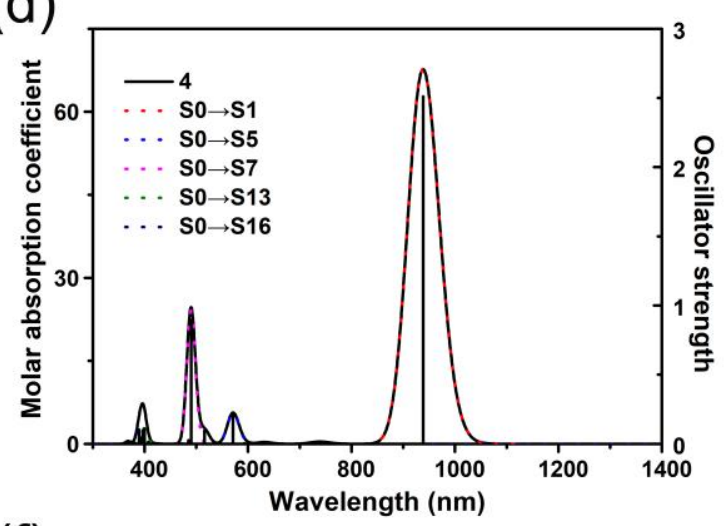

(f)

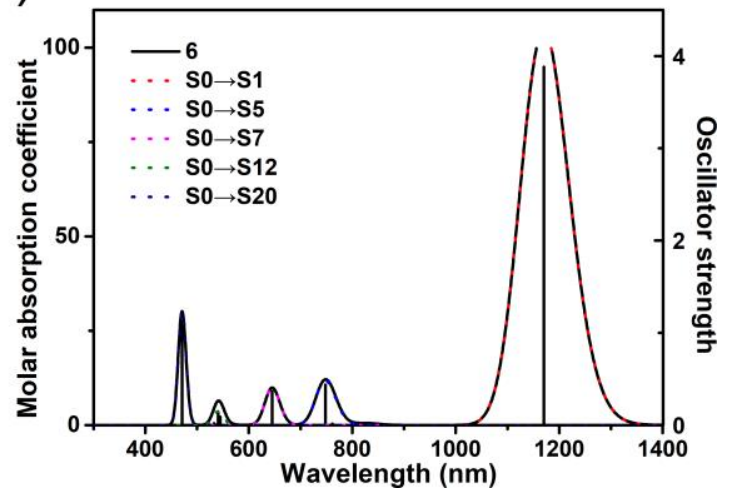

Figure S4. Calculated UV-vis absorption (a-f) of energy-minimized calculated (Gaussian) monomer 1 and arrays 2-6. The unit for molar absorption coefficient is $10^{4} \mathrm{~L} / \mathrm{mol} / \mathrm{cm}$. Calculation was performed at B3LYP/6-31G* level with Gaussian 09. 
Table S1. The optimized geometries and electronic properties of the studied conjugated oligomers.

\begin{tabular}{cccccc}
\hline arrays & $\theta^{\mathrm{a}}\left(^{\mathrm{o}}\right)$ & $\mathrm{HOMO}^{\mathrm{b}}(\mathrm{eV})$ & $\mathrm{LUMO}^{\mathrm{c}}(\mathrm{eV})$ & $\mathrm{Eg}^{\mathrm{d}}(\mathrm{eV})$ & $\lambda_{\mathrm{abs}}{ }^{\mathrm{e}}(\mathrm{nm})$ \\
\hline $\boldsymbol{\alpha - \alpha ( 2 )}$ & 4.31 & -5.33 & -3.24 & 2.09 & $\mathbf{6 2 0 . 9}$ \\
$\boldsymbol{\alpha}-\boldsymbol{\beta}$ & 2.98 & -5.37 & -3.1 & 2.27 & 621.3 \\
$\boldsymbol{\beta}-\boldsymbol{\beta}$ & 4.51 & -5.36 & -2.96 & 2.41 & 597.3 \\
$\boldsymbol{\alpha}-\boldsymbol{m}$ & 15.21 & -5.88 & -3.4 & 2.48 & 553.8 \\
$\boldsymbol{\beta}-\boldsymbol{m}$ & 39.04 & -5.89 & -3.16 & 2.73 & 507.1 \\
$\boldsymbol{m}-\boldsymbol{m}$ & 73.19 & -6.07 & -3.56 & 2.51 & 550 \\
$\boldsymbol{\alpha - \alpha - \alpha ( 3 )}$ & 5.04 & -5.1 & -3.42 & 1.68 & $\mathbf{8 0 9 . 1}$ \\
$\boldsymbol{\alpha - \alpha - \alpha - \alpha ( 4 )}$ & 3.33 & -4.98 & -3.5 & 1.48 & $\mathbf{9 3 7 . 8}$ \\
$\boldsymbol{\alpha - \alpha - \alpha - \alpha - \alpha ( 5 )}$ & 5.15 & -4.91 & -3.56 & 1.35 & $\mathbf{1 0 7 7 . 4}$ \\
$\boldsymbol{\alpha - \alpha - \alpha - \alpha - \alpha - \alpha ( 6 )}$ & 1.17 & -4.87 & -3.59 & 1.28 & $\mathbf{1 1 6 9 . 3}$ \\
\hline
\end{tabular}

${ }^{a}$ Dihedral angels between adjacent dipyrrin planes. ${ }^{b}$ The HOMO energy level. ${ }^{c}$ The LUMO energy level. ${ }^{\mathrm{d}}$ The calculated band gap. ${ }^{\mathrm{e}}$ Calculated absorption maxima. 
Table S2. Selected electronic excitation energies (eV) and oscillator strengths (f), configurations of the low-lying excited states of the possible ethene linked BODIPY dimer calculated by TDDFT//B3LYP/6-31G(d, p), based on the optimized ground state geometries.

\begin{tabular}{|c|c|c|c|c|c|}
\hline \multirow{2}{*}{ dimers } & \multirow{2}{*}{$\begin{array}{l}\text { Electronic } \\
\text { transition }\end{array}$} & \multicolumn{4}{|c|}{ TD//B3LYP/6-31G(d, p) } \\
\hline & & Energy/ eV ${ }^{[a]}$ & $f^{[\mathrm{b}]}$ & Composition $^{[\mathrm{c}]}$ & $\mathrm{CI}^{[\mathrm{d}]}$ \\
\hline \multirow[t]{7}{*}{$\alpha-\alpha$} & $\mathrm{S} 0 \rightarrow \mathrm{S} 1$ & $1.9967 \mathrm{eV} \quad 620.94 \mathrm{~nm}$ & 1.6688 & $\mathrm{HOMO} \rightarrow$ LUMO & 0.7122 \\
\hline & & & & $\mathrm{HOMO} \leftarrow$ LUMO & 0.1071 \\
\hline & $\mathrm{S} 0 \rightarrow \mathrm{S} 2$ & $2.4941 \mathrm{eV} \quad 497.11 \mathrm{~nm}$ & 0.0000 & HOMO - $1 \rightarrow$ LUMO & 0.4966 \\
\hline & & & & $\mathrm{HOMO} \rightarrow \mathrm{LUMO}+1$ & 0.5004 \\
\hline & $\mathrm{S} 0 \rightarrow \mathrm{S} 3$ & $2.9629 \mathrm{eV} \quad 418.46 \mathrm{~nm}$ & 0.0000 & HOMO - $1 \rightarrow$ LUMO & 0.4655 \\
\hline & & & & $\mathrm{HOMO} \rightarrow \mathrm{LUMO}+1$ & 0.4580 \\
\hline & & & & HOMO $\leftarrow$ LUMO & 0.1071 \\
\hline \multirow[t]{7}{*}{$\alpha-\beta$} & $\mathrm{S} 0 \rightarrow \mathrm{S} 1$ & $1.9955 \mathrm{eV} \quad 621.32 \mathrm{~nm}$ & 0.6912 & $\mathrm{HOMO} \rightarrow$ LUMO & 0.6980 \\
\hline & & & & $\mathrm{HOMO} \rightarrow \mathrm{LUMO}+1$ & 0.1132 \\
\hline & $\mathrm{S} 0 \rightarrow \mathrm{S} 2$ & $2.4680 \mathrm{eV} 502.36 \mathrm{~nm}$ & 0.8123 & HOMO - $1 \rightarrow$ LUMO & 0.2493 \\
\hline & & & & HOMO $\rightarrow$ LUMO & 0.1132 \\
\hline & & & & $\mathrm{HOMO} \rightarrow \mathrm{LUMO}+1$ & 0.6508 \\
\hline & $\mathrm{S} 0 \rightarrow \mathrm{S} 3$ & $2.9844 \mathrm{eV} \quad 415.44 \mathrm{~nm}$ & 0.1981 & HOMO - $1 \rightarrow$ LUMO & 0.6182 \\
\hline & & & & HOMO -2 $\rightarrow$ LUMO & 0.1922 \\
\hline \multirow[t]{6}{*}{$\alpha-m$} & $\mathrm{~S} 0 \rightarrow \mathrm{S} 1$ & $2.2389 \mathrm{eV} 553.78 \mathrm{~nm}$ & 0.7818 & HOMO $\rightarrow$ LUMO & 0.6737 \\
\hline & $\mathrm{S} 0 \rightarrow \mathrm{S} 2$ & $2.3092 \mathrm{eV} 536.91 \mathrm{~nm}$ & 0.1997 & HOMO $\rightarrow$ LUMO & 0.1818 \\
\hline & & & & $\mathrm{HOMO}-1 \rightarrow \mathrm{LUMO}+1$ & 0.1300 \\
\hline & & & & HOMO -1 $\rightarrow$ LUMO & 0.6695 \\
\hline & $\mathrm{S} 0 \rightarrow \mathrm{S} 3$ & $2.9534 \mathrm{eV} \quad 419.80 \mathrm{~nm}$ & 0.0447 & HOMO $-2 \rightarrow$ LUMO & 0.5425 \\
\hline & & & & HOMO - $1 \rightarrow$ LUMO +1 & 0.4298 \\
\hline \multirow[t]{5}{*}{$\beta-\beta$} & $\mathrm{S} 0 \rightarrow \mathrm{S} 1$ & $2.0756 \mathrm{eV} 597.34 \mathrm{~nm}$ & 0.8413 & HOMO $\rightarrow$ LUMO & 0.7048 \\
\hline & $\mathrm{S} 0 \rightarrow \mathrm{S} 2$ & $2.2703 \mathrm{eV} 546.11 \mathrm{~nm}$ & 0.0432 & HOMO - $1 \rightarrow$ LUMO & 0.1216 \\
\hline & & & & $\mathrm{HOMO} \rightarrow \mathrm{LUMO}+1$ & 0.6878 \\
\hline & $\mathrm{S} 0 \rightarrow \mathrm{S} 3$ & $2.9969 \mathrm{eV} \quad 413.71 \mathrm{~nm}$ & 0.0817 & HOMO - $\rightarrow$ LUMO & 0.6328 \\
\hline & & & & HOMO $-1 \rightarrow$ LUMO +1 & 0.2064 \\
\hline \multirow[t]{6}{*}{$\beta-m$} & $\mathrm{~S} 0 \rightarrow \mathrm{S} 1$ & $2.4448 \mathrm{eV} 507.14 \mathrm{~nm}$ & 0.9989 & HOMO $\rightarrow$ LUMO & 0.4664 \\
\hline & & & & HOMO - $1 \rightarrow$ LUMO & 0.5117 \\
\hline & $\mathrm{S} 0 \rightarrow \mathrm{S} 2$ & $2.4831 \mathrm{eV} \quad 499.31 \mathrm{~nm}$ & 0.1600 & HOMO $\rightarrow$ LUMO & 0.5030 \\
\hline & & & & HOMO - $1 \rightarrow$ LUMO & 0.4614 \\
\hline & $\mathrm{S} 0 \rightarrow \mathrm{S} 3$ & $2.8572 \mathrm{eV} \quad 433.94 \mathrm{~nm}$ & 0.1062 & HOMO $-2 \rightarrow$ LUMO & 0.2036 \\
\hline & & & & $\mathrm{HOMO} \rightarrow \mathrm{LUMO}+1$ & 0.5632 \\
\hline \multirow[t]{6}{*}{$m-m$} & $\mathrm{~S} 0 \rightarrow \mathrm{S} 1$ & $2.2542 \mathrm{eV} 550.02 \mathrm{~nm}$ & 0.0882 & HOMO $-1 \rightarrow$ LUMO & 0.6927 \\
\hline & & & & $\mathrm{HOMO} \rightarrow \mathrm{LUMO}+1$ & 0.1409 \\
\hline & $\mathrm{S} 0 \rightarrow \mathrm{S} 2$ & $2.2613 \mathrm{eV} 548.29 \mathrm{~nm}$ & 0.1694 & HOMO $\rightarrow$ LUMO & 0.6909 \\
\hline & & & & $\mathrm{HOMO}-1 \rightarrow \mathrm{LUMO}+1$ & 0.1447 \\
\hline & $\mathrm{S} 0 \rightarrow \mathrm{S} 3$ & $2.9329 \mathrm{eV} 422.73 \mathrm{~nm}$ & 0.0222 & HOMO -4 $\rightarrow$ LUMO & 0.2899 \\
\hline & & & & HOMO $-2 \rightarrow$ LUMO & 0.5813 \\
\hline
\end{tabular}

[a] Only the selected low-lying excited states are presented. [b] Oscillator strength. [c] Only the main configurations are presented. [d] The CI coefficients are in absolute values. 
Table S3. Selected electronic excitation energies (eV) and oscillator strengths (f), configurations of the low-lying excited states of the monomer $\mathbf{1}$ and oligomers $\mathbf{2 - 6}$ calculated by TDDFT//B3LYP/6-31G(d, p), based on the optimized ground state geometries.

\begin{tabular}{|c|c|c|c|c|c|}
\hline \multirow{2}{*}{ arrays } & \multirow{2}{*}{$\begin{array}{c}\text { Electronic } \\
\text { transition }\end{array}$} & \multicolumn{4}{|c|}{ TD//B3LYP/6-31G (d, p) } \\
\hline & & Energy/ eV ${ }^{[a]}$ & $f^{[\mathrm{b}]}$ & Composition $^{[\mathrm{c}]}$ & $\mathrm{CI}^{[\mathrm{d}]}$ \\
\hline \multirow[t]{5}{*}{1} & $\mathrm{~S} 0 \rightarrow \mathrm{S} 1$ & $3.0528 \mathrm{eV} \quad 406.13 \mathrm{~nm}$ & 0.5093 & $\mathrm{HOMO} \rightarrow$ LUMO & 0.6815 \\
\hline & & & & $\mathrm{HOMO} \leftarrow$ LUMO & 0.1063 \\
\hline & $\mathrm{S} 0 \rightarrow \mathrm{S} 2$ & $3.6580 \mathrm{eV} \quad 338.94 \mathrm{~nm}$ & 0.1878 & HOMO - $1 \rightarrow$ LUMO & 0.6753 \\
\hline & & & & HOMO $\rightarrow$ LUMO & 0.2083 \\
\hline & $\mathrm{S} 0 \rightarrow \mathrm{S} 3$ & $3.8184 \mathrm{eV} \quad 324.70 \mathrm{~nm}$ & 0.0367 & HOMO -1 $\rightarrow$ LUMO & 0.7034 \\
\hline \multirow[t]{6}{*}{2} & $\mathrm{~S} 0 \rightarrow \mathrm{S} 1$ & $1.9967 \mathrm{eV} \quad 620.94 \mathrm{~nm}$ & 1.6688 & HOMO $\rightarrow$ LUMO & 0.7122 \\
\hline & & & & $\mathrm{HOMO} \leftarrow$ LUMO & 0.1071 \\
\hline & $\mathrm{S} 0 \rightarrow \mathrm{S} 2$ & $2.4941 \mathrm{eV} \quad 497.11 \mathrm{~nm}$ & 0.0000 & HOMO -1 $\rightarrow$ LUMO & 0.4966 \\
\hline & & & & $\mathrm{HOMO} \rightarrow \mathrm{LUMO}+1$ & 0.5004 \\
\hline & $\mathrm{S} 0 \rightarrow \mathrm{S} 3$ & $2.9629 \mathrm{eV} \quad 418.46 \mathrm{~nm}$ & 0.0000 & HOMO -1 $\rightarrow$ LUMO & 0.4655 \\
\hline & & & & $\mathrm{HOMO} \rightarrow \mathrm{LUMO}+1$ & 0.4580 \\
\hline \multirow[t]{5}{*}{3} & $\mathrm{~S} 0 \rightarrow \mathrm{S} 1$ & $1.5325 \mathrm{eV} \quad 809.05 \mathrm{~nm}$ & 2.0032 & HOMO $\rightarrow$ LUMO & 0.7109 \\
\hline & $\mathrm{S} 0 \rightarrow \mathrm{S} 2$ & $1.9472 \mathrm{eV} \quad 636.74 \mathrm{~nm}$ & 0.0004 & HOMO -1 $\rightarrow$ LUMO & 0.4856 \\
\hline & & & & $\mathrm{HOMO} \rightarrow \mathrm{LUMO}+1$ & 0.5089 \\
\hline & $\mathrm{S} 0 \rightarrow \mathrm{S} 3$ & $2.3614 \mathrm{eV} 525.04 \mathrm{~nm}$ & 0.6041 & HOMO -1 $\rightarrow$ LUMO & 0.5141 \\
\hline & & & & $\mathrm{HOMO} \rightarrow \mathrm{LUMO}+1$ & 0.4923 \\
\hline \multirow[t]{5}{*}{4} & $\mathrm{~S} 0 \rightarrow \mathrm{S} 1$ & $1.3221 \mathrm{eV} \quad 937.81 \mathrm{~nm}$ & 2.5106 & HOMO $\rightarrow$ LUMO & 0.7069 \\
\hline & $\mathrm{S} 0 \rightarrow \mathrm{S} 2$ & $1.6795 \mathrm{eV} \quad 738.24 \mathrm{~nm}$ & 0.0145 & HOMO -1 $\rightarrow$ LUMO & 0.4952 \\
\hline & & & & $\mathrm{HOMO} \rightarrow \mathrm{LUMO}+1$ & 0.5005 \\
\hline & $\mathrm{S} 0 \rightarrow \mathrm{S} 3$ & $1.9636 \mathrm{eV} \quad 631.41 \mathrm{~nm}$ & 0.0116 & HOMO - $1 \rightarrow$ LUMO & 0.5017 \\
\hline & & & & $\mathrm{HOMO} \rightarrow \mathrm{LUMO}+1$ & 0.4979 \\
\hline \multirow[t]{5}{*}{5} & $\mathrm{~S} 0 \rightarrow \mathrm{S} 1$ & $1.1508 \mathrm{eV} 1077.36 \mathrm{~nm}$ & 3.2789 & HOMO $\rightarrow$ LUMO & 0.7042 \\
\hline & $\mathrm{S} 0 \rightarrow \mathrm{S} 2$ & $1.4559 \mathrm{eV} 851.58 \mathrm{~nm}$ & 0.0001 & HOMO -1 $\rightarrow$ LUMO & 0.4799 \\
\hline & & & & $\mathrm{HOMO} \rightarrow \mathrm{LUMO}+1$ & 0.5094 \\
\hline & $\mathrm{S} 0 \rightarrow \mathrm{S} 3$ & $1.6823 \mathrm{eV} 736.98 \mathrm{~nm}$ & 0.0556 & HOMO - $1 \rightarrow$ LUMO & 0.5189 \\
\hline & & & & $\mathrm{HOMO} \rightarrow \mathrm{LUMO}+1$ & 0.4904 \\
\hline \multirow[t]{6}{*}{6} & $\mathrm{~S} 0 \rightarrow \mathrm{S} 1$ & $1.0603 \mathrm{eV} 1169.32 \mathrm{~nm}$ & 3.8811 & HOMO $\rightarrow$ LUMO & 0.6996 \\
\hline & & & & $\mathrm{HOMO}-1 \rightarrow \mathrm{LUMO}+1$ & 0.1101 \\
\hline & $\mathrm{S} 0 \rightarrow \mathrm{S} 2$ & $1.3410 \mathrm{eV} 924.60 \mathrm{~nm}$ & 0.0001 & HOMO -1 $\rightarrow$ LUMO & 0.4756 \\
\hline & & & & $\mathrm{HOMO} \rightarrow \mathrm{LUMO}+1$ & 0.5105 \\
\hline & $\mathrm{S} 0 \rightarrow \mathrm{S} 3$ & $1.5015 \mathrm{eV} 825.73 \mathrm{~nm}$ & 0.0197 & HOMO - $1 \rightarrow$ LUMO & 0.5202 \\
\hline & & & & $\mathrm{HOMO} \rightarrow \mathrm{LUMO}+1$ & 0.4863 \\
\hline
\end{tabular}

[a] Only the selected low-lying excited states are presented. [b] Oscillator strength. [c] Only the main configurations are presented. [d] The CI coefficients are in absolute values. 


\section{Synthesis and characterization}

BODIPY $\mathbf{1}$ was synthesized according to the literature. ${ }^{11}$ BODIPYs $\mathbf{1 C l}$ and $\mathbf{1} \mathbf{C l}_{\mathbf{2}}$ were synthesized from BODIPY $\mathbf{1}$ according to literature. ${ }^{12}$

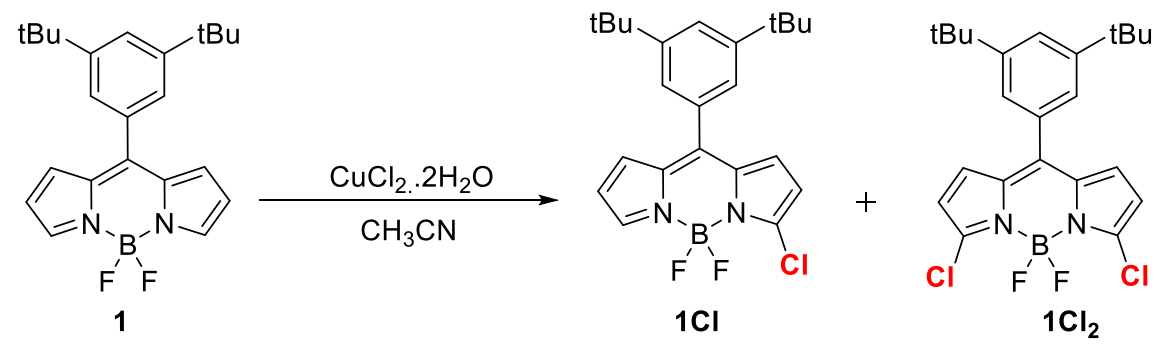

A mixture of BODIPY $1(380 \mathrm{mg}, 1 \mathrm{mmol})$ and $\mathrm{CuCl}_{2} 2 \mathrm{H}_{2} \mathrm{O}(1.02 \mathrm{~g}, 6 \mathrm{mmol})$ in $\mathrm{CH}_{3} \mathrm{CN}$ $\left(20 \mathrm{~mL}\right.$ ) was stirred under air at $80^{\circ} \mathrm{C}$ in oil bath for $40 \mathrm{~min}$. After cooling down to room temperature, the reaction mixture was poured in dichloromethane $(50 \mathrm{~mL})$. The mixture was washed with water $(3 \times 50 \mathrm{~mL})$. The organic layer was collected, dried over anhydrous $\mathrm{Na}_{2} \mathrm{SO}_{4}$, filtered and evaporated under vacuum. The residue was purified through column chromatography on silica using petroleum ether/ dichloromethane $(10: 3$, $\mathrm{v} / \mathrm{v}$ ) to afford $\mathbf{1 C l}$ as a purple solid in $48 \%$ (199 $\mathrm{mg}$ ) yield and $\mathbf{1} \mathbf{C l} 2$ as a purple solid in $31 \%$ (139 mg) yield. The yields were calculated based on BODIPY 1.

1Cl: ${ }^{1} \mathrm{H}$ NMR (500 MHz, $\left.\mathrm{CDCl}_{3}\right) \delta 7.94(\mathrm{~s}, 1 \mathrm{H}), 7.63$ (t, $\left.J=2.5 \mathrm{~Hz}, 1 \mathrm{H}\right), 7.36$ (d, $J=1.5$ Hz, 2H), $6.96-6.88(\mathrm{~m}, 2 \mathrm{H}), 6.57(\mathrm{dd}, J=4.0,2.5 \mathrm{~Hz}, 1 \mathrm{H}), 6.44(\mathrm{~d}, J=5.0 \mathrm{~Hz}, 1 \mathrm{H})$, 1.38 (s, 18H). ${ }^{13} \mathrm{C}$ NMR $\left(126 \mathrm{MHz}, \mathrm{CDCl}_{3}\right) \delta 151.3,147.3,144.0,134.8,134.1,132.5$, $131.9,131.6,125.2,124.9,118.7,118.2,77.3,77.0,76.8,35.0,31.4$. HRMS (MALDI-TOF) m/z: [M] ${ }^{+}$Calcd for $\mathrm{C}_{23} \mathrm{H}_{26} \mathrm{BClF}_{2} \mathrm{~N}_{2}$ 414.1840, Found 414.1840.

1Cl2: ${ }^{1} \mathrm{H}$ NMR $\left(400 \mathrm{MHz}, \mathrm{CDCl}_{3}\right) \delta 7.62(\mathrm{t}, J=2.4 \mathrm{~Hz}, 1 \mathrm{H}), 7.31(\mathrm{~d}, J=1.6 \mathrm{~Hz}, 2 \mathrm{H})$, $6.87(\mathrm{~d}, J=4.0 \mathrm{~Hz}, 2 \mathrm{H}), 6.45(\mathrm{~d}, J=4.0 \mathrm{~Hz}, 2 \mathrm{H}), 1.37$ (s, 18H). ${ }^{13} \mathrm{C}$ NMR $(101 \mathrm{MHz}$, $\left.\mathrm{CDCl}_{3}\right) \delta 1512,144.4,133.9,131.8,131.7,125.2,125.0,118.7,77.3,77.0,76.7,35.0$, 31.4. HRMS (MALDI-TOF) m/z: $[\mathrm{M}]^{+}$Calcd for $\mathrm{C}_{23} \mathrm{H}_{25} \mathrm{BCl}_{2} \mathrm{~F}_{2} \mathrm{~N}_{2}$ 448.1450, Found 448.1451 . 
Synthesis of dimer 2.

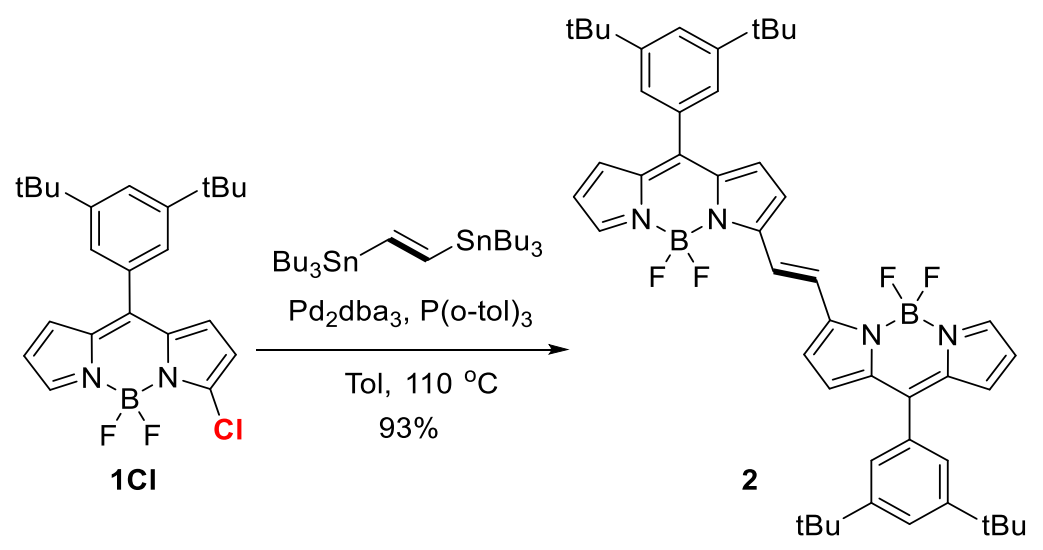

A Schlenk tube containing $\mathbf{1 C l}(92 \quad \mathrm{mg}, \quad 0.22 \quad \mathrm{mmol})$, trans-1,2-bis(tri-n-butylstannyl)-ethylene (53 $\mu \mathrm{L}, 0.1 \mathrm{mmol}), \mathrm{Pd}_{2} \mathrm{dba}_{3}(4.6 \mathrm{mg}, 0.005$ $\mathrm{mmol})$ and tris(2-methylphenyl)phosphine $(6 \mathrm{mg}, 0.02 \mathrm{mmol})$ was evacuated and refilled with Ar three times. Degassed toluene $(5 \mathrm{~mL})$ was then added via syringe and the solution was stirred in oil bath at $110{ }^{\circ} \mathrm{C}$ for $6 \mathrm{~h}$ under $\mathrm{Ar}$ atmosphere. The solution was then poured into a saturated ammonium chloride aqueous solution and was extracted with dichloromethane three times. The combined organic layers were washed with water, dried over $\mathrm{Na}_{2} \mathrm{SO}_{4}$, filtered and evaporated under vacuum. The residue was purified using chromatography ( (73 mg) yield. The yield was calculated based on ESn. ${ }^{1} \mathrm{H}$ NMR $\left(500 \mathrm{MHz}, \mathrm{CD}_{2} \mathrm{Cl}_{2}\right) \delta$ $7.84(\mathrm{~d}, J=6.0 \mathrm{~Hz}, 4 \mathrm{H}), 7.63(\mathrm{~s}, 2 \mathrm{H}), 7.39$ (d, $J=1.7 \mathrm{~Hz}, 4 \mathrm{H}), 7.14$ (d, $J=4.6 \mathrm{~Hz}, 2 \mathrm{H})$, $6.99(\mathrm{~d}, J=4.6 \mathrm{~Hz}, 2 \mathrm{H}), 6.89(\mathrm{~d}, J=4.1 \mathrm{~Hz}, 2 \mathrm{H}), 6.55(\mathrm{dd}, J=4.0,1.8 \mathrm{~Hz}, 2 \mathrm{H}), 1.35(\mathrm{~s}$, $36 \mathrm{H}) .{ }^{13} \mathrm{C}$ NMR $\left(101 \mathrm{MHz}, \mathrm{CD}_{2} \mathrm{Cl}_{2}\right) \delta 154.4,151.1,145.7,142.0,137.8,135.2,133.2$, $132.0,130.0,126.1,125.3,124.8,118.4,118.2,54.0,53.7,53.4,53.2,52.9,34.9,31.1$. HRMS (MALDI-TOF) m/z: [M] ${ }^{+}$Calcd for $\mathrm{C}_{48} \mathrm{H}_{54} \mathrm{~B}_{2} \mathrm{~F}_{4} \mathrm{~N}_{4}$ 784.4465, Found 784.4464. 
Synthesis of $\mathbf{1 S n}$.

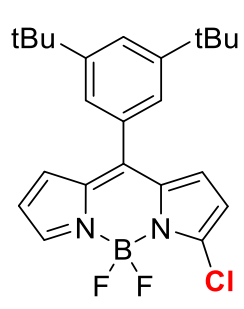

1CI

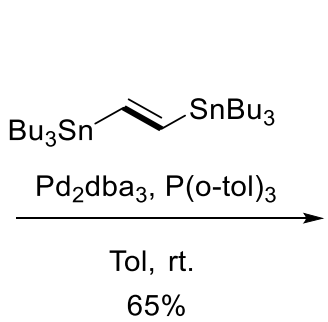

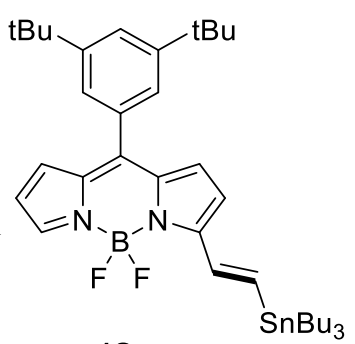

1Sn

A Schlenk tube containing $\mathbf{1 C l} \quad(42 \quad \mathrm{mg}, \quad 0.1 \quad \mathrm{mmol})$, trans-1,2-bis(tri-n-butylstannyl)-ethylene (159 $\mu \mathrm{L}, 0.3 \mathrm{mmol}), \mathrm{Pd}_{2} \mathrm{dba}_{3}$ (4.6 mg, 0.005 $\mathrm{mmol})$ and tris(2-methylphenyl)phosphine $(6 \mathrm{mg}, 0.02 \mathrm{mmol})$ was evacuated and refilled with Ar three times. Degassed toluene $(5 \mathrm{~mL})$ was then added via syringe and the solution was stirred at room temperature for $8 \mathrm{~h}$ under Ar atmosphere. The solution was then poured into a saturated ammonium chloride aqueous solution and was extracted with dichloromethane three times. The combined organic layers were washed with water, dried over $\mathrm{Na}_{2} \mathrm{SO}_{4}$, filtered and evaporated under vacuum. The residue was purified using chromatography (silica gel, hexane/ $\mathrm{CH}_{2} \mathrm{Cl}_{2}=4 / 1$, v/v) to afford 1Sn as purple oil in $65 \%$ (45 mg) yield. The yield was calculated based on 1Cl. ${ }^{1} \mathrm{H} \mathrm{NMR}\left(500 \mathrm{MHz}, \mathrm{CD}_{2} \mathrm{Cl}_{2}\right) \delta$ $7.68(\mathrm{~s}, 1 \mathrm{H}), 7.55(\mathrm{~s}, 1 \mathrm{H}), 7.52-7.39(\mathrm{~m}, 2 \mathrm{H}), 7.31(\mathrm{~s}, 2 \mathrm{H}), 6.87(\mathrm{~d}, J=4.5 \mathrm{~Hz}, 1 \mathrm{H}), 6.83$ $(\mathrm{d}, J=4.5 \mathrm{~Hz}, 1 \mathrm{H}), 6.74(\mathrm{~d}, J=3.6 \mathrm{~Hz}, 1 \mathrm{H}), 6.43(\mathrm{~d}, J=1.9 \mathrm{~Hz}, 1 \mathrm{H}), 1.54-1.47(\mathrm{~m}, 6 \mathrm{H})$, $1.29(\mathrm{~s}, 18 \mathrm{H}), 1.19(\mathrm{~d}, J=13.9 \mathrm{~Hz}, 6 \mathrm{H}), 1.01-0.96(\mathrm{~m}, 6 \mathrm{H}), 0.84(\mathrm{t}, J=7.3 \mathrm{~Hz}, 9 \mathrm{H}) .{ }^{13} \mathrm{C}$ NMR (101 MHz, $\left.\mathrm{CD}_{2} \mathrm{Cl}_{2}\right) \delta 157.3,150.9,147.6,145.8,139.8,136.3,134.1,133.4,132.4$, $128.3,125.2,124.5,117.0,54.0,53.7,53.4,53.1,52.9,34.8,31.1,29.1,27.3,13.5,9.9$. HRMS (APCI) m/z: Calcd for $\mathrm{C}_{37} \mathrm{H}_{55} \mathrm{BFN}_{2} \mathrm{Sn}[\mathrm{M}-\mathrm{F}]^{+}$677.3463, Found 677.3456. 
Synthesis of trimer 3 .

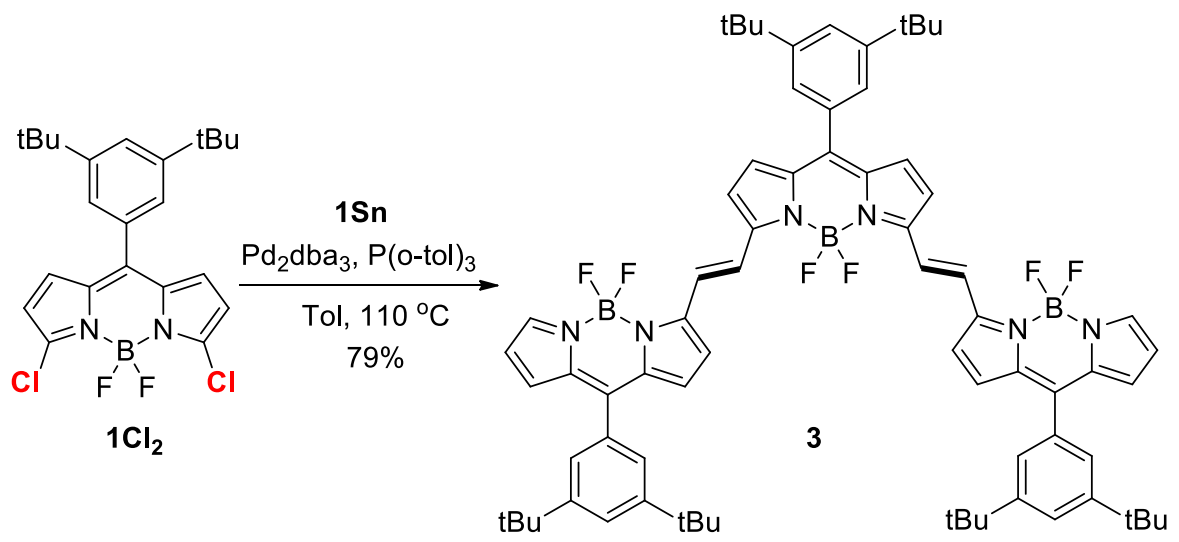

A Schlenk tube containing $\mathbf{1 C \mathbf { l } _ { 2 }}(44 \mathrm{mg}, 0.1 \mathrm{mmol}), \mathbf{1 S n}(154 \mathrm{mg}, 0.22 \mathrm{mmol}), \mathrm{Pd}_{2}(\mathrm{dba})_{3}$ (4.6 $\mathrm{mg}, 0.005 \mathrm{mmol})$ and tris(2-methylphenyl)phosphine $(6 \mathrm{mg}, 0.02 \mathrm{mmol})$ was evacuated and refilled with Ar three times. Degassed toluene $(5 \mathrm{~mL})$ was then added via syringe and the solution was stirred in oil bath at $110 \mathrm{C}^{\mathrm{o}}$ for $6 \mathrm{~h}$ under Ar atmosphere. The solution was then poured into a saturated ammonium chloride aqueous solution and was extracted with dichloromethane three times. The combined organic layers were washed with water, dried over $\mathrm{Na}_{2} \mathrm{SO}_{4}$, filtered and evaporated under vacuum. The residue was purified using chromatography (silica gel, hexane $/ \mathrm{CH}_{2} \mathrm{Cl}_{2}=3 / 1$, v/v) to afford $\mathbf{3}$ as a black power in 79\% (94 mg) yield. The yield was calculated based on $\mathbf{1 C l}$. ${ }^{1} \mathrm{H}$ NMR (500 MHz, $\left.\mathrm{CD}_{2} \mathrm{Cl}_{2}\right) \delta 7.98(\mathrm{~d}, J=15.0 \mathrm{~Hz}, 3 \mathrm{H}), 7.91(\mathrm{~d}, J=15.0 \mathrm{~Hz}, 3 \mathrm{H}), 7.68$ $(\mathrm{t}, J=2.0 \mathrm{~Hz}, 3 \mathrm{H}), 7.45(\mathrm{dd}, J=5.0,2.5 \mathrm{~Hz}, 6 \mathrm{H}), 7.28(\mathrm{~d}, J=5.0 \mathrm{~Hz}, 2 \mathrm{H}), 7.23(\mathrm{~d}, J=$ $3.5 \mathrm{~Hz}, 2 \mathrm{H}), 7.06(\mathrm{~d}, J=5.0 \mathrm{~Hz}, 2 \mathrm{H}), 6.99(\mathrm{~d}, J=4.5 \mathrm{~Hz}, 2 \mathrm{H}), 6.94(\mathrm{~d}, J=5.0 \mathrm{~Hz}, 2 \mathrm{H})$, $6.61(\mathrm{dd}, J=3.5,3.0 \mathrm{~Hz}, 2 \mathrm{H}), 1.41(\mathrm{~d}, J=5.0 \mathrm{~Hz}, 54 \mathrm{H}) .{ }^{13} \mathrm{C} \mathrm{NMR}\left(101 \mathrm{MHz}, \mathrm{CDCl}_{3}\right) \delta$ $156.7,155.2$, 153.1, 153.0, 147.1, 143.7, 143.6, 140.4, 139.9, 137.1, 135.2, 135.1, 133.9, $132.4,131.6,128.5,127.5,127.3,127.2,126.7,126.5,120.7,120.5,120.0,55.9,55.6$, 55.3, 55.1, 54.8, 36.8, 33.0. HRMS (MALDI-TOF) $\mathrm{m} / \mathrm{z}:[\mathrm{M}]^{+}$Calcd for $\mathrm{C}_{73} \mathrm{H}_{81} \mathrm{~B}_{3} \mathrm{~F}_{6} \mathrm{~N}_{6}$ 1188.6726, Found 1188.6699. 
Synthesis of $\alpha$-Chlorination $\mathbf{2 C l}$.

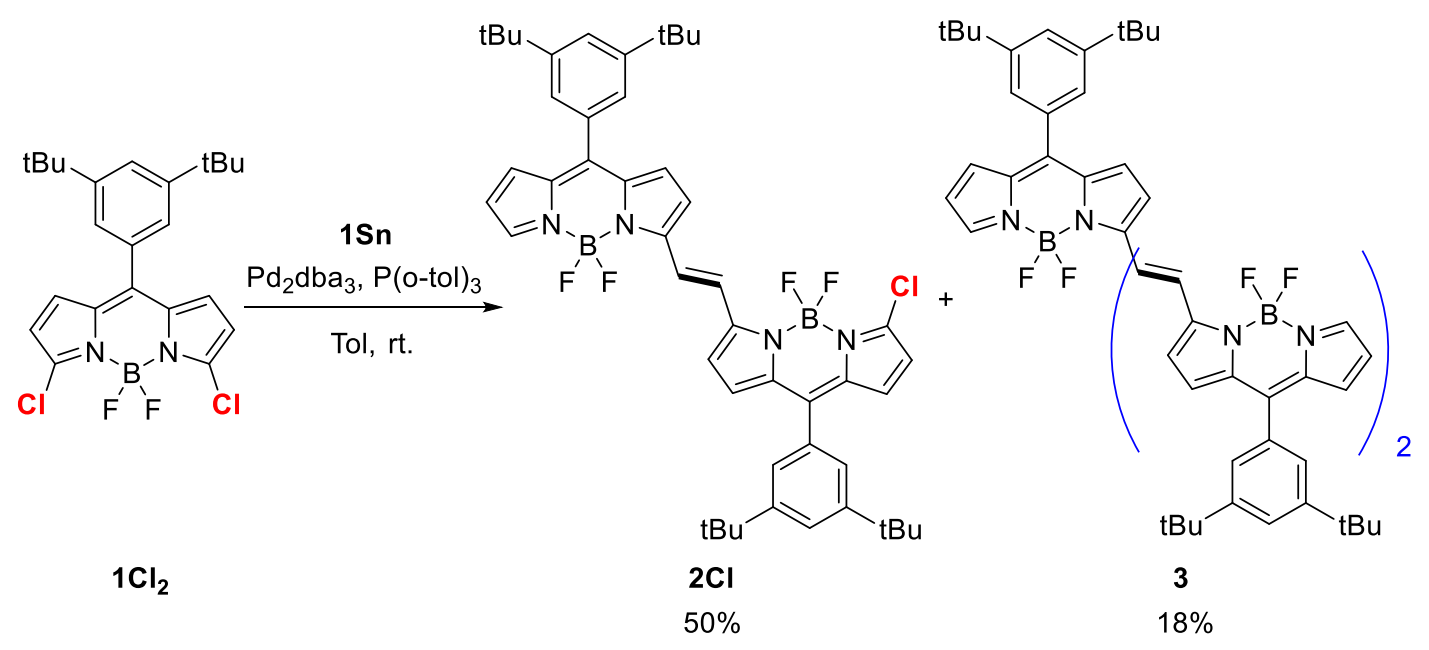

A Schlenk tube containing $\mathbf{1} \mathbf{C l}_{2}(67 \mathrm{mg}, 0.15 \mathrm{mmol}), \mathbf{1 S n}(70 \mathrm{mg}, 0.1 \mathrm{mmol}), \mathrm{Pd}_{2}(\mathrm{dba})_{3}$ (4.6 $\mathrm{mg}, 0.005 \mathrm{mmol})$ and tris(2-methylphenyl)phosphine $(6 \mathrm{mg}, 0.02 \mathrm{mmol})$ was evacuated and refilled with Ar three times. Degassed toluene $(5 \mathrm{~mL})$ was then added via syringe and the solution was stirred at room temperature for $12 \mathrm{~h}$ under Ar atmosphere. The solution was then poured into a saturated ammonium chloride aqueous solution and was extracted with dichloromethane three times. The combined organic layers were washed with water, dried over $\mathrm{Na}_{2} \mathrm{SO}_{4}$, filtered and evaporated under vacuum. The residue was purified using chromatography (silica gel, hexane $/ \mathrm{CH}_{2} \mathrm{Cl}_{2}=3 / 1$, v/v) to afford 2Cl as a black power in 50\% (41 mg) yield. The yield was calculated based on 1Sn. ${ }^{1} \mathrm{H}$ NMR $\left(500 \mathrm{MHz}, \mathrm{CD}_{2} \mathrm{Cl}_{2}\right) \delta 7.90(\mathrm{~d}, J=6.1 \mathrm{~Hz}, 3 \mathrm{H}), 7.68(\mathrm{dd}, J=3.3,1.7 \mathrm{~Hz}, 2 \mathrm{H})$, $7.45(\mathrm{~d}, J=1.8 \mathrm{~Hz}, 2 \mathrm{H}), 7.40(\mathrm{~d}, J=1.8 \mathrm{~Hz}, 2 \mathrm{H}), 7.22(\mathrm{t}, J=5.2 \mathrm{~Hz}, 2 \mathrm{H}), 7.05(\mathrm{~d}, J=$ $4.6 \mathrm{~Hz}, 1 \mathrm{H}), 7.02(\mathrm{~d}, J=4.6 \mathrm{~Hz}, 1 \mathrm{H}), 6.95(\mathrm{~d}, J=4.0 \mathrm{~Hz}, 1 \mathrm{H}), 6.88(\mathrm{~d}, J=4.2 \mathrm{~Hz}, 1 \mathrm{H})$, $6.61(\mathrm{dd}, J=4.1,1.9 \mathrm{~Hz}, 1 \mathrm{H}), 6.49(\mathrm{~d}, J=4.3 \mathrm{~Hz}, 1 \mathrm{H}), 1.40(\mathrm{~d}, J=3.8 \mathrm{~Hz}, 36 \mathrm{H}) .{ }^{13} \mathrm{C}$ NMR (101 MHz, $\left.\mathrm{CD}_{2} \mathrm{Cl}_{2}\right) \delta 154.9,154.2,145.8,143.9,142.2,141.6,137.9,137.7,135.3$, $134.3,133.2,132.5,131.9,130.2,130.1,126.6,125.9,125.3,125.3,124.9,121.2,118.9$, 118.6, 118.2, 117.9, 54.0, 53.7, 53.4, 53.2, 52.9, 34.9, 31.1. HRMS (MALDI-TOF) m/z: $[\mathrm{M}]^{+}$Calcd for $\mathrm{C}_{48} \mathrm{H}_{53} \mathrm{~B}_{2} \mathrm{ClF}_{4} \mathrm{~N}_{4}$ 818.4076, Found 818.4073.

Trimer 3 was also isolated as a black power in $18 \%(21 \mathrm{mg})$ yield in the above reaction. 
Synthesis of dichloroBODIPY $\mathbf{2} \mathrm{Cl}_{\mathbf{2}}, \mathbf{3} \mathrm{Cl}_{\mathbf{2}}$ and $\mathbf{4} \mathrm{Cl}_{\mathbf{2}}$.

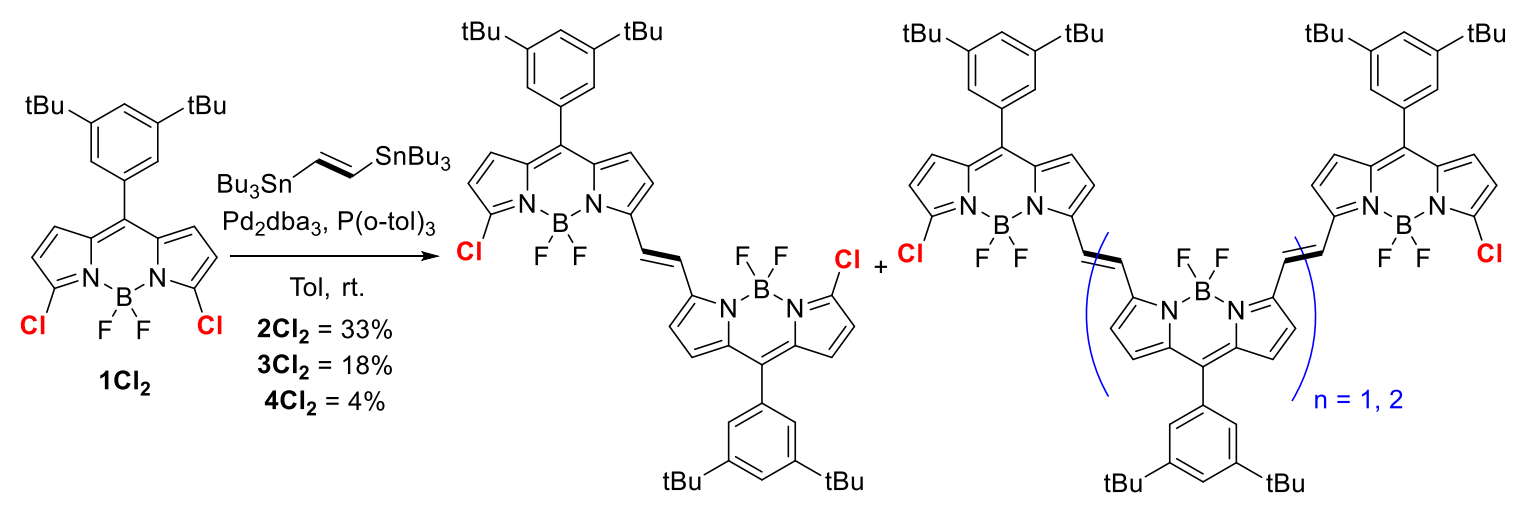

A Schlenk tube containing $\quad \mathbf{1 C l}_{\mathbf{2}} \quad(98 \quad \mathrm{mg}, \quad 0.22 \quad \mathrm{mmol})$,

trans-1,2-bis(tri-n-butylstannyl)-ethylene (53 $\mu \mathrm{L}, 0.1 \mathrm{mmol}), \mathrm{Pd}_{2} \mathrm{dba}_{3}(4.6 \mathrm{mg}, 0.005$ $\mathrm{mmol})$ and tris(2-methylphenyl)phosphine $(6 \mathrm{mg}, 0.02 \mathrm{mmol})$ was evacuated and refilled with Ar three times. Degassed toluene $(5 \mathrm{~mL})$ was then added via syringe and the solution was stirred at room temperature for $24 \mathrm{~h}$ under Ar atmosphere. The solution was then poured into a saturated ammonium chloride aqueous solution and was extracted with dichloromethane three times. The combined organic layers were washed with water, dried over $\mathrm{Na}_{2} \mathrm{SO}_{4}$, filtered and evaporated under vacuum. The residue was purified using chromatography (silica gel, hexane $/ \mathrm{CH}_{2} \mathrm{Cl}_{2}=3 / 1$, v/v) to afford $\mathbf{2} \mathbf{C l}_{2}$ as a black power in $33 \%$ (28 $\mathrm{mg}$ ) yield and $\mathbf{3} \mathbf{C l}_{2}$ as a black power in $18 \%(20 \mathrm{mg})$ yield. The yields were calculated based on $\mathbf{1} \mathbf{C l}_{2}$.

2Cl2: ${ }^{1} \mathrm{H}$ NMR $\left(400 \mathrm{MHz}, \mathrm{CDCl}_{3}\right) \delta 7.93(\mathrm{~s}, 2 \mathrm{H}), 7.62(\mathrm{t}, J=1.8 \mathrm{~Hz}, 2 \mathrm{H}), 7.34(\mathrm{~d}, J=$ $1.8 \mathrm{~Hz}, 4 \mathrm{H}), 7.23(\mathrm{~d}, J=4.7 \mathrm{~Hz}, 2 \mathrm{H}), 6.96(\mathrm{~d}, J=4.6 \mathrm{~Hz}, 2 \mathrm{H}), 6.84(\mathrm{~d}, J=4.1 \mathrm{~Hz}, 2 \mathrm{H})$, $6.44(\mathrm{~d}, J=4.2 \mathrm{~Hz}, 2 \mathrm{H}), 1.38(\mathrm{~s}, 36 \mathrm{H}) \cdot{ }^{13} \mathrm{C} \mathrm{NMR}\left(101 \mathrm{MHz}, \mathrm{CDCl}_{3}\right) \delta 178.5,1611$, $154.7,154.6,134.9,132.0,116.3,114.7,112.9,112.8,107.2,102.1,94.4,77.4,77.1,76.7$, 64.7, 64.7, 46.0, 45.6, 34.1, 31.5, 12.7, 12.6. HRMS (MALDI-TOF) m/z: [M] ${ }^{+}$Calcd for $\mathrm{C}_{48} \mathrm{H}_{52} \mathrm{~B}_{2} \mathrm{Cl}_{2} \mathrm{~F}_{4} \mathrm{~N}_{4}$ 852.3686, Found 852.3684.

3Cl $2:{ }^{1} \mathrm{H}$ NMR (400 MHz, $\left.\mathrm{CD}_{2} \mathrm{Cl}_{2}\right) \delta 8.02(\mathrm{~d}, J=24.0 \mathrm{~Hz}, 2 \mathrm{H}), 7.93(\mathrm{~d}, J=16.0 \mathrm{~Hz}, 2 \mathrm{H})$, $7.69(\mathrm{~s}, 3 \mathrm{H}), 7.46-7.41(\mathrm{~m}, 6 \mathrm{H}), 7.32(\mathrm{~d}, J=4.0 \mathrm{~Hz}, 2 \mathrm{H}), 7.28(\mathrm{~d}, J=4.0 \mathrm{~Hz}, 2 \mathrm{H}), 7.05$ $(\mathrm{d}, J=4.0 \mathrm{~Hz}, 2 \mathrm{H}), 7.02(\mathrm{~d}, J=4.0 \mathrm{~Hz}, 2 \mathrm{H}), 6.90(\mathrm{~d}, J=4.0 \mathrm{~Hz}, 2 \mathrm{H}), 6.51(\mathrm{~d}, J=4.0 \mathrm{~Hz}$, 
$2 \mathrm{H}), 1.43(\mathrm{~d}, J=8.0 \mathrm{~Hz}, 54 \mathrm{H}) .{ }^{13} \mathrm{C}$ NMR $\left(126 \mathrm{MHz}, \mathrm{CD}_{2} \mathrm{Cl}_{2}\right) \delta 155.6,153.6,151.6$, $143.8,142.3,141.7,139.0,138.2,134.7,133.5,133.0,132.2,130.9,130.2,127.5,125.8$, 125.7, 125.2, 119.4, 118.2, 54.2, 54.0, 53.8, 53.6, 53.4, 35.3, 31.5. HRMS (MALDI-TOF) $\mathrm{m} / \mathrm{z}$ : $[\mathrm{M}]^{+}$Calcd for $\mathrm{C}_{73} \mathrm{H}_{79} \mathrm{~B}_{3} \mathrm{Cl}_{2} \mathrm{~F}_{6} \mathrm{~N}_{6}$ 1256.5983, Found 1256.6013.

Small amount of $\mathbf{4} \mathbf{C l}_{\mathbf{2}}$ was also isolated as a black power in $4 \%(5 \mathrm{mg})$ yield, which was only characterized by high resolution HRMS. HRMS (MALDI-TOF) m/z: [M] $]^{+}$Calcd for $\mathrm{C}_{98} \mathrm{H}_{106} \mathrm{~B}_{4} \mathrm{Cl}_{2} \mathrm{~F}_{8} \mathrm{~N}_{8}$ 1661.8207, Found 1661.8189.

Synthesis of tetramer 4.
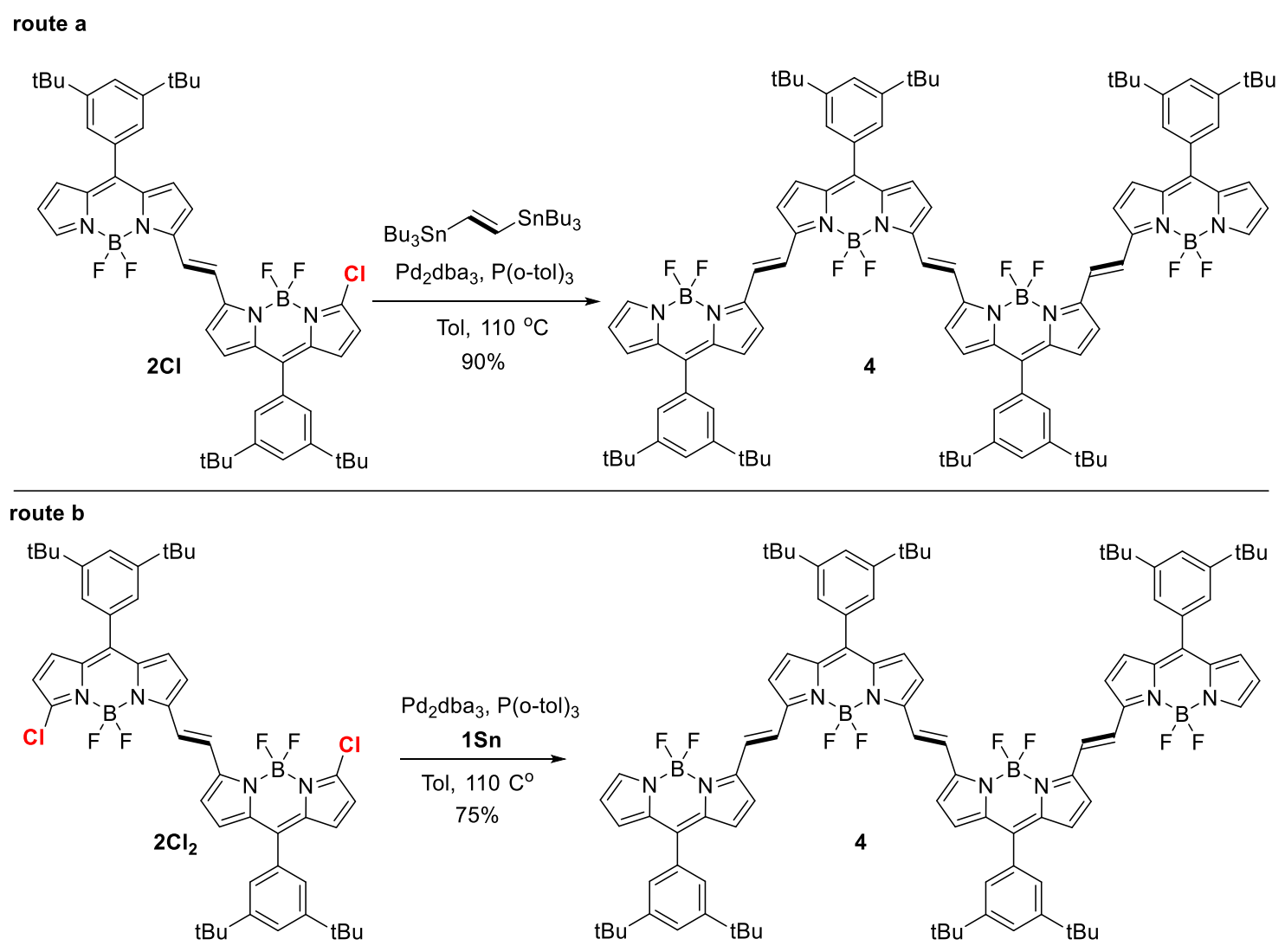

route a: A Schlenk tube containing 2 Cl $\quad\left(\begin{array}{lllll}180 & \mathrm{mg}, & 0.22 & \mathrm{mmol}\end{array}\right)$, trans-1,2-bis(tri-n-butylstannyl)-ethylene $(53 \mu \mathrm{L}, 0.1 \mathrm{mmol}), \mathrm{Pd}_{2} \mathrm{dba}_{3}(4.6 \mathrm{mg}, 0.005$ mmol) and tris(2-methylphenyl)phosphine $(6 \mathrm{mg}, 0.02 \mathrm{mmol})$ was evacuated and refilled with Ar three times. Degassed toluene $(5 \mathrm{~mL})$ was then added via syringe and the solution was stirred in oil bath at $110{ }^{\circ} \mathrm{C}$ for $6 \mathrm{~h}$ under Ar atmosphere. The reaction was then 
stopped, cooled to room temperature, and poured into a saturated ammonium chloride aqueous solution. The mixture was extracted with dichloromethane three times. The combined organic layers were washed with water, dried over $\mathrm{Na}_{2} \mathrm{SO}_{4}$, filtered and evaporated under vacuum. The residue was purified using chromatography (silica gel, hexane $\left./ \mathrm{CH}_{2} \mathrm{Cl}_{2}=3 / 1, \mathrm{v} / \mathrm{v}\right)$ to afford 4 as a black power in $90 \%(71 \mathrm{mg}$ ) yield. The yield was calculated based on ESn.

route b: A Schlenk tube containing $\mathbf{2} \mathbf{C l}_{\mathbf{2}}(85 \mathrm{mg}, 0.1 \mathrm{mmol})$, $\mathbf{1 S n}(154 \mathrm{mg}, 0.22 \mathrm{mmol})$, $\mathrm{Pd}_{2}(\mathrm{dba})_{3}(4.6 \mathrm{mg}, 0.005 \mathrm{mmol})$ and tris(2-methylphenyl)phosphine $(6 \mathrm{mg}, 0.02 \mathrm{mmol})$ was evacuated and refilled with Ar three times. Degassed toluene $(5 \mathrm{~mL})$ was then added via syringe and the solution was stirred in oil bath at $110 \mathrm{C}^{\circ}$ for $6 \mathrm{~h}$ under Ar atmosphere. The reaction was then stopped, cooled to room temperature, and poured into a saturated ammonium chloride aqueous solution. The mixture was extracted with dichloromethane three times. The combined organic layers were washed with water, dried over $\mathrm{Na}_{2} \mathrm{SO}_{4}$, filtered and evaporated under vacuum. The residue was purified using chromatography (silica gel, hexane/ $\mathrm{CH}_{2} \mathrm{Cl}_{2}=3 / 1$, v/v) to afford 4 as a black power in $75 \%$ (119 mg) yield. The yield was calculated based on $\mathbf{2} \mathbf{C l}_{\mathbf{2}}$.

${ }^{1} \mathrm{H}$ NMR $\left(500 \mathrm{MHz}, \mathrm{CD}_{2} \mathrm{Cl}_{2}\right) \delta 7.92(\mathrm{t}, J=10.0 \mathrm{~Hz}, 4 \mathrm{H}), 7.83(\mathrm{~d}, J=20.0 \mathrm{~Hz}, 4 \mathrm{H}), 7.61$ $(\mathrm{d}, J=2.0 \mathrm{~Hz}, 4 \mathrm{H}), 7.38(\mathrm{dd}, J=3.0,5.0 \mathrm{~Hz}, 8 \mathrm{H}), 7.22(\mathrm{~d}, J=2.0 \mathrm{~Hz}, 2 \mathrm{H}), 7.18(\mathrm{~d}, J=$ $4.6 \mathrm{~Hz}, 2 \mathrm{H}), 7.14$ (d, $J=4.5 \mathrm{~Hz}, 2 \mathrm{H}), 7.00(\mathrm{~d}, J=4.5 \mathrm{~Hz}, 2 \mathrm{H}), 6.89$ (dd, $J=5.0,4.5 \mathrm{~Hz}$, $4 \mathrm{H}), 6.87(\mathrm{~d}, J=5.0 \mathrm{~Hz}, 2 \mathrm{H}), 6.51(\mathrm{dd}, J=5.0,2.5 \mathrm{~Hz}, 2 \mathrm{H}), 1.35(\mathrm{~d}, J=5.0 \mathrm{~Hz}, 72 \mathrm{H})$. ${ }^{13} \mathrm{C}$ NMR (101 MHz, $\left.\mathrm{CD}_{2} \mathrm{Cl}_{2}\right) \delta 154.9,153.7,153.0,151.1,151.1,145.1,141.6,141.3$, 138.7, 138.5, 138.0, 135.1, 133.3, 133.2, 132.0, 130.4, 130.2, 129.6, 126.7, 126.1, 125.3, $125.3,124.7,124.5,119.0,118.7,118.7,118.0,116.6,54.0,53.7,53.4,53.2,52.9,34.9$, 31.1. HRMS (MALDI-TOF) m/z: $[\mathrm{M}]^{+}$Calcd for $\mathrm{C}_{98} \mathrm{H}_{108} \mathrm{~B}_{4} \mathrm{~F}_{8} \mathrm{~N}_{8}$ 1592.8999, Found 1592.8964. 
Synthesis of pentamer $\mathbf{5}$.

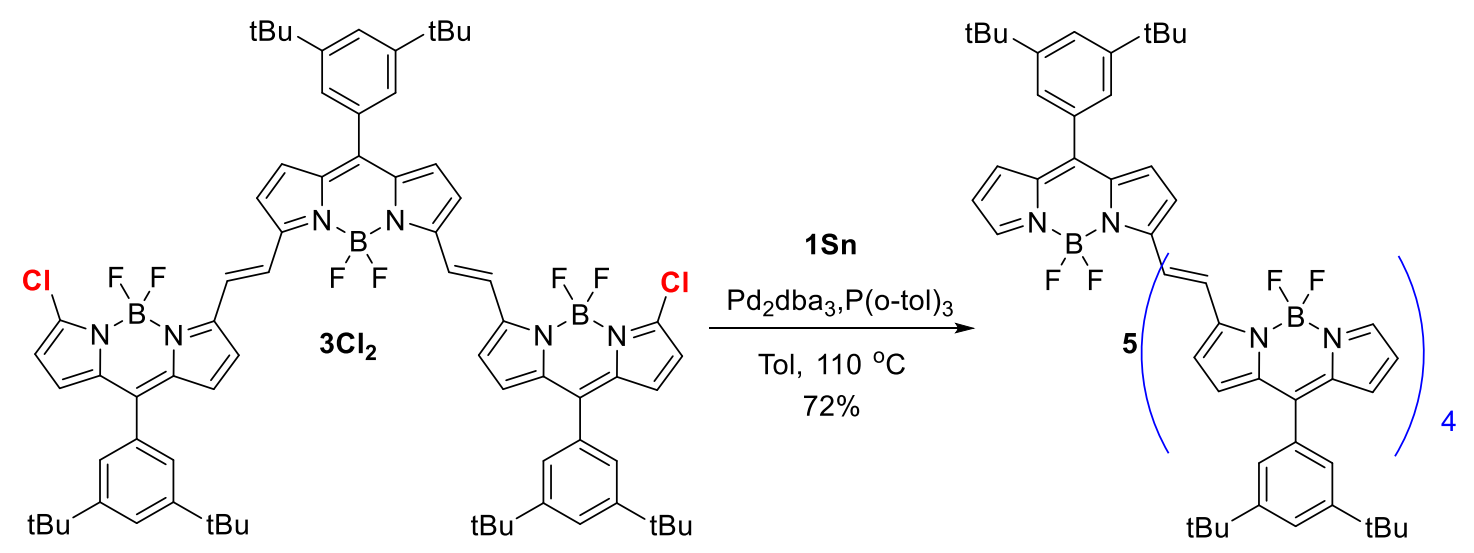

A Schlenk tube containing $\mathbf{3} \mathbf{C l}_{2}(126 \mathrm{mg}, 0.1 \mathrm{mmol})$, 1Sn (154 mg, $\left.0.22 \mathrm{mmol}\right), \mathrm{Pd}_{2} \mathrm{dba}_{3}$ (4.6 $\mathrm{mg}, 0.005 \mathrm{mmol})$ and tris $(2-$ methylphenyl)phosphine $(6 \mathrm{mg}, 0.02 \mathrm{mmol})$ was evacuated and refilled with nitrogen three times. Degassed toluene $(5 \mathrm{~mL})$ was then added via syringe and the solution was stirred in oil bath at $110{ }^{\circ} \mathrm{C}$ for $6 \mathrm{~h}$ under nitrogen atmosphere. The reaction was then stopped, cooled to room temperature, and poured into a saturated ammonium chloride aqueous solution. The mixture was extracted with dichloromethane three times. The combined organic layers were washed with water, dried over $\mathrm{Na}_{2} \mathrm{SO}_{4}$, filtered and evaporated under vacuum. The residue was purified using chromatography (silica gel, hexane/ $\mathrm{CH}_{2} \mathrm{Cl}_{2}=2 / 1$, v/v) to afford 5 as a black power in $72 \%\left(162 \mathrm{mg}\right.$ ) yield. The yield was calculated based on $\mathbf{3 C l}_{2} .{ }^{1} \mathrm{H}$ NMR (500 MHz, $\left.\mathrm{CD}_{2} \mathrm{Cl}_{2}\right) \delta 7.90(\mathrm{~d}, J=10.0 \mathrm{~Hz}, 5 \mathrm{H}), 7.80(\mathrm{~d}, J=17.5 \mathrm{~Hz}, 5 \mathrm{H}), 7.58(\mathrm{~s}, 5 \mathrm{H}), 7.35(\mathrm{~d}, J=$ $5.0 \mathrm{~Hz}, 10 \mathrm{H}), 7.23(\mathrm{~d}, J=4.0 \mathrm{~Hz}, 4 \mathrm{H}), 7.19(\mathrm{~d}, J=4.0 \mathrm{~Hz}, 2 \mathrm{H}), 7.13(\mathrm{~d}, J=4.0 \mathrm{~Hz}, 2 \mathrm{H})$, $6.97(\mathrm{~d}, J=4.0 \mathrm{~Hz}, 2 \mathrm{H}), 6.91(\mathrm{~d}, J=5.0 \mathrm{~Hz}, 3 \mathrm{H}), 6.88(\mathrm{~d}, J=5.0 \mathrm{~Hz}, 3 \mathrm{H}), 6.84(\mathrm{~d}, J=$ $3.0 \mathrm{~Hz}, 2 \mathrm{H}), 6.50(\mathrm{~d}, J=4.5 \mathrm{~Hz}, 2 \mathrm{H}), 1.31(\mathrm{~d}, J=5.0 \mathrm{~Hz}, 90 \mathrm{H})$. The ${ }^{13} \mathrm{C}$ NMR spectrum was not available due to its poor solubility. HRMS (MALDI-TOF) m/z: [M] ${ }^{+}$Calcd for $\mathrm{C}_{123} \mathrm{H}_{135} \mathrm{~B}_{5} \mathrm{~F}_{10} \mathrm{~N}_{10}$ 1997.1234, Found 1997.1240. 
Synthesis of $\alpha^{-}$-chlorinated $\mathbf{3 C l}$.

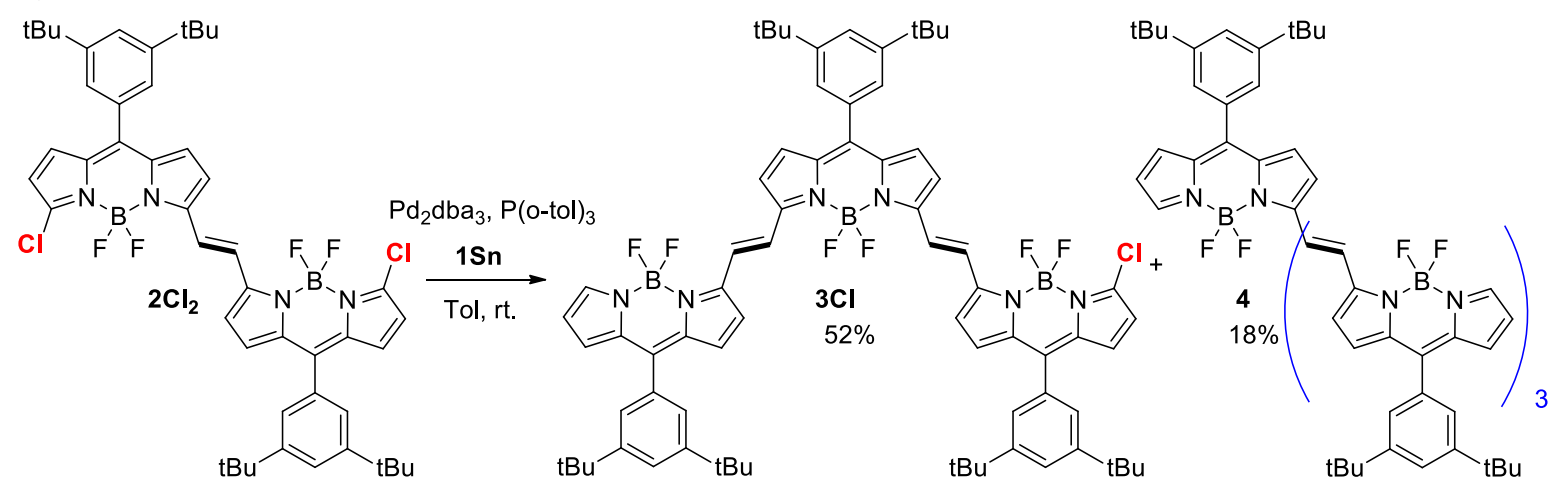

A Schlenk tube containing $\mathbf{2 C \mathbf { l } _ { 2 }}(128 \mathrm{mg}, 0.15 \mathrm{mmol}), \mathbf{1 S n}(70 \mathrm{mg}, 0.1 \mathrm{mmol}), \mathrm{Pd}_{2}(\mathrm{dba})_{3}$ (4.6 $\mathrm{mg}, 0.005 \mathrm{mmol})$ and tris(2-methylphenyl)phosphine $(6 \mathrm{mg}, 0.02 \mathrm{mmol})$ was evacuated and refilled with Ar three times. Degassed toluene $(5 \mathrm{~mL})$ was then added via syringe and the solution was stirred at room temperature for $12 \mathrm{~h}$ under Ar atmosphere. The solution was then poured into a saturated ammonium chloride aqueous solution and was extracted with dichloromethane three times. The combined organic layers were washed with water, dried over $\mathrm{Na}_{2} \mathrm{SO}_{4}$, filtered and evaporated under vacuum. The residue was purified using chromatography (silica gel, hexane/ $\mathrm{CH}_{2} \mathrm{Cl}_{2}=3 / 1$, v/v) to afford $\mathbf{3 C l}$ as a black power in 52\% (64 mg) yield. The yield was calculated based on 1Sn. ${ }^{1} \mathrm{H}$ NMR (500 MHz, $\left.\mathrm{CD}_{2} \mathrm{Cl}_{2}\right) \delta 8.01(\mathrm{~d}, J=10.0 \mathrm{~Hz}, 1 \mathrm{H}), 7.99(\mathrm{~d}, J=10.0 \mathrm{~Hz}, 2 \mathrm{H}), 7.91$ $(\mathrm{d}, J=19.3 \mathrm{~Hz}, 4 \mathrm{H}), 7.68(\mathrm{dd}, J=1.5,2.0 \mathrm{~Hz}, 3 \mathrm{H}), 7.45(\mathrm{~d}, J=5.0 \mathrm{~Hz}, 2 \mathrm{H}), 7.44(\mathrm{~d}, J=$ $5.0 \mathrm{~Hz}, 2 \mathrm{H}), 7.41(\mathrm{~d}, J=1.5 \mathrm{~Hz}, 2 \mathrm{H}), 7.29(\mathrm{dd}, J=5.0,10.0 \mathrm{~Hz}, 2 \mathrm{H}), 7.26$ (d, $J=4.5 \mathrm{~Hz}$, $1 \mathrm{H}), 7.23(\mathrm{~d}, J=4.6 \mathrm{~Hz}, 1 \mathrm{H}), 7.06(\mathrm{~d}, J=4.5 \mathrm{~Hz}, 1 \mathrm{H}), 7.03(\mathrm{~d}, J=4.6 \mathrm{~Hz}, 1 \mathrm{H}), 6.99$ (t, $J$ $=4.4 \mathrm{~Hz}, 2 \mathrm{H}), 6.94(\mathrm{~d}, J=3.9 \mathrm{~Hz}, 1 \mathrm{H}), 6.87(\mathrm{~d}, J=4.1 \mathrm{~Hz}, 1 \mathrm{H}), 6.61(\mathrm{dd}, J=4.1,1.9 \mathrm{~Hz}$, $1 \mathrm{H}), 6.48(\mathrm{~d}, J=4.2 \mathrm{~Hz}, 1 \mathrm{H}), 1.42-1.39(\mathrm{~m}, 54 \mathrm{H}) .{ }^{13} \mathrm{C} \mathrm{NMR}\left(101 \mathrm{MHz}, \mathrm{CD}_{2} \mathrm{Cl}_{2}\right) \delta$ $155.3,154.7,153.4,153.0,151.2,151.1,145.2,143.4,141.9,141.8,141.2,138.5,138.0$, $137.8,135.2$, 134.3, 133.3, 133.2, 132.6, 131.9, 131.8, 130.6, 130.4, 129.8, 127.1, 126.5, $125.7,125.3,125.3,125.3,124.8,124.8,124.6,119.1,119.0,118.9,118.6,118.1,117.7$, 54.0, 53.7, 53.4, 53.2, 52.9, 34.9, 31.1. HRMS (MALDI-TOF) m/z: $[\mathrm{M}]^{+}$Calcd for $\mathrm{C}_{73} \mathrm{H}_{80} \mathrm{~B}_{3} \mathrm{ClF}_{6} \mathrm{~N}_{6}$ 1222.6341, Found 1222.6294.

Tetramer 4 was also isolated as a black power in 18\% (29 $\mathrm{mg}$ ) yield in the above reaction. 
Synthesis of hexamer 6.

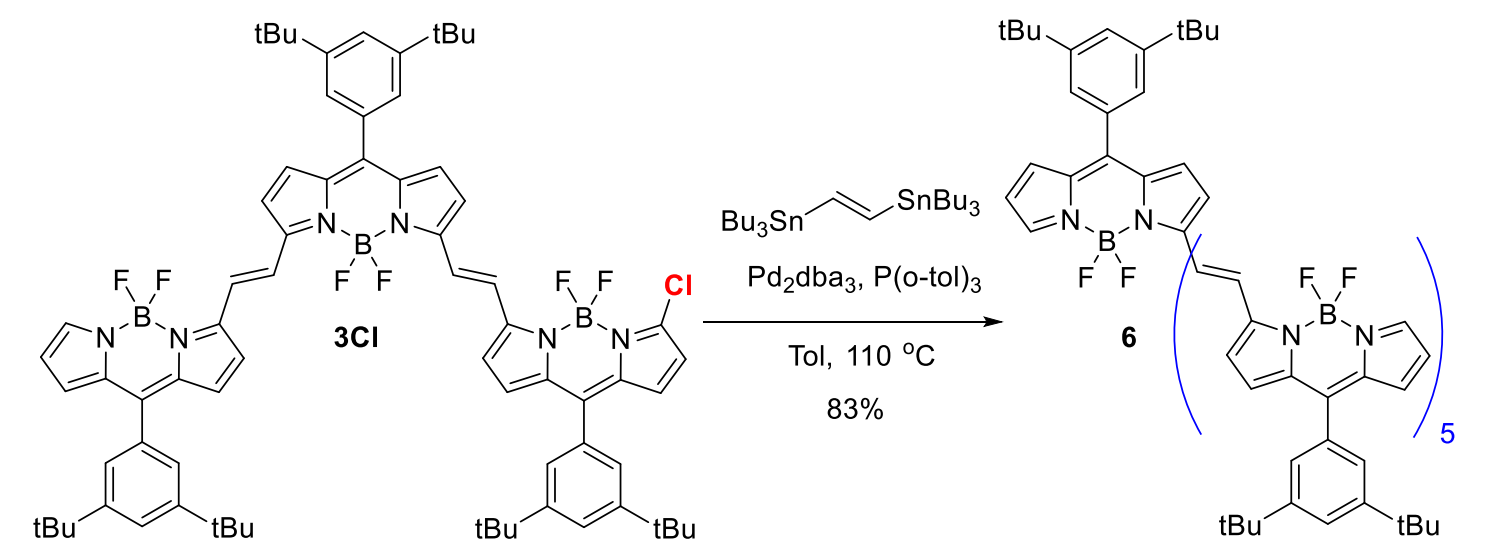

A Schlenk tube containing 3Cl (269 $\mathrm{mg}, 0.22 \quad \mathrm{mmol})$,

trans-1,2-bis(tri-n-butylstannyl)-ethylene (53 $\mu \mathrm{L}, 0.1 \mathrm{mmol}), \mathrm{Pd}_{2} \mathrm{dba}_{3}$ (4.6 mg, 0.005 mmol) and tris(2-methylphenyl)phosphine (6 mg, $0.02 \mathrm{mmol})$ was evacuated and refilled with Ar three times. Degassed toluene ( $5 \mathrm{~mL}$ ) was then added via syringe and the solution was stirred in oil bath at $110{ }^{\circ} \mathrm{C}$ for $6 \mathrm{~h}$ under $\mathrm{Ar}$ atmosphere. The reaction was then stopped, cooled to room temperature, and poured into a saturated ammonium chloride aqueous solution. The mixture was extracted with dichloromethane three times. The combined organic layers were washed with water, dried over $\mathrm{Na}_{2} \mathrm{SO}_{4}$, filtered and evaporated under vacuum. The residue was purified using chromatography (silica gel, hexane $\left./ \mathrm{CH}_{2} \mathrm{Cl}_{2}=2 / 1, \mathrm{v} / \mathrm{v}\right)$ to afford 6 as a black power in $83 \%(199 \mathrm{mg}$ ) yield. The yield was calculated based on ESn. ${ }^{1} \mathrm{H}$ NMR $\left(500 \mathrm{MHz}, \mathrm{CD}_{2} \mathrm{Cl}_{2}\right) \delta 7.88(\mathrm{~d}, J=12.5 \mathrm{~Hz}, 6 \mathrm{H})$, $7.80(\mathrm{~d}, J=18.0 \mathrm{~Hz}, 6 \mathrm{H}), 7.58(\mathrm{~s}, 6 \mathrm{H}), 7.34(\mathrm{~d}, J=10.0 \mathrm{~Hz}, 12 \mathrm{H}), 7.23(\mathrm{~d}, J=3.0 \mathrm{~Hz}$, 2H), $7.20(\mathrm{~d}, J=3.5 \mathrm{~Hz}, 4 \mathrm{H}), 7.16(\mathrm{~d}, J=5.0 \mathrm{~Hz}, 2 \mathrm{H}), 7.13(\mathrm{~d}, J=3.5 \mathrm{~Hz}, 2 \mathrm{H}), 6.99$ (d, $J=4.0 \mathrm{~Hz}, 2 \mathrm{H}), 6.97(\mathrm{~d}, J=2.5 \mathrm{~Hz}, 2 \mathrm{H}), 6.91(\mathrm{~d}, J=4.5 \mathrm{~Hz}, 3 \mathrm{H}), 6.86(\mathrm{~d}, J=7.5 \mathrm{~Hz}$, $3 \mathrm{H}), 6.83(\mathrm{~d}, J=3.5 \mathrm{~Hz}, 2 \mathrm{H}), 6.50(\mathrm{~d}, J=5.0 \mathrm{~Hz}, 2 \mathrm{H}), 1.31(\mathrm{~d}, J=5.0 \mathrm{~Hz}, 108 \mathrm{H})$. The ${ }^{13} \mathrm{C}$ NMR spectrum was not available due to its poor solubility. HRMS (MALDI-TOF) $\mathrm{m} / \mathrm{z}:[\mathrm{M}]^{+}$Calcd for $\mathrm{C}_{148} \mathrm{H}_{162} \mathrm{~B}_{6} \mathrm{~F}_{12} \mathrm{~N}_{12}$ 2401.3486, Found 2401.3487. 


\section{Crystal data}
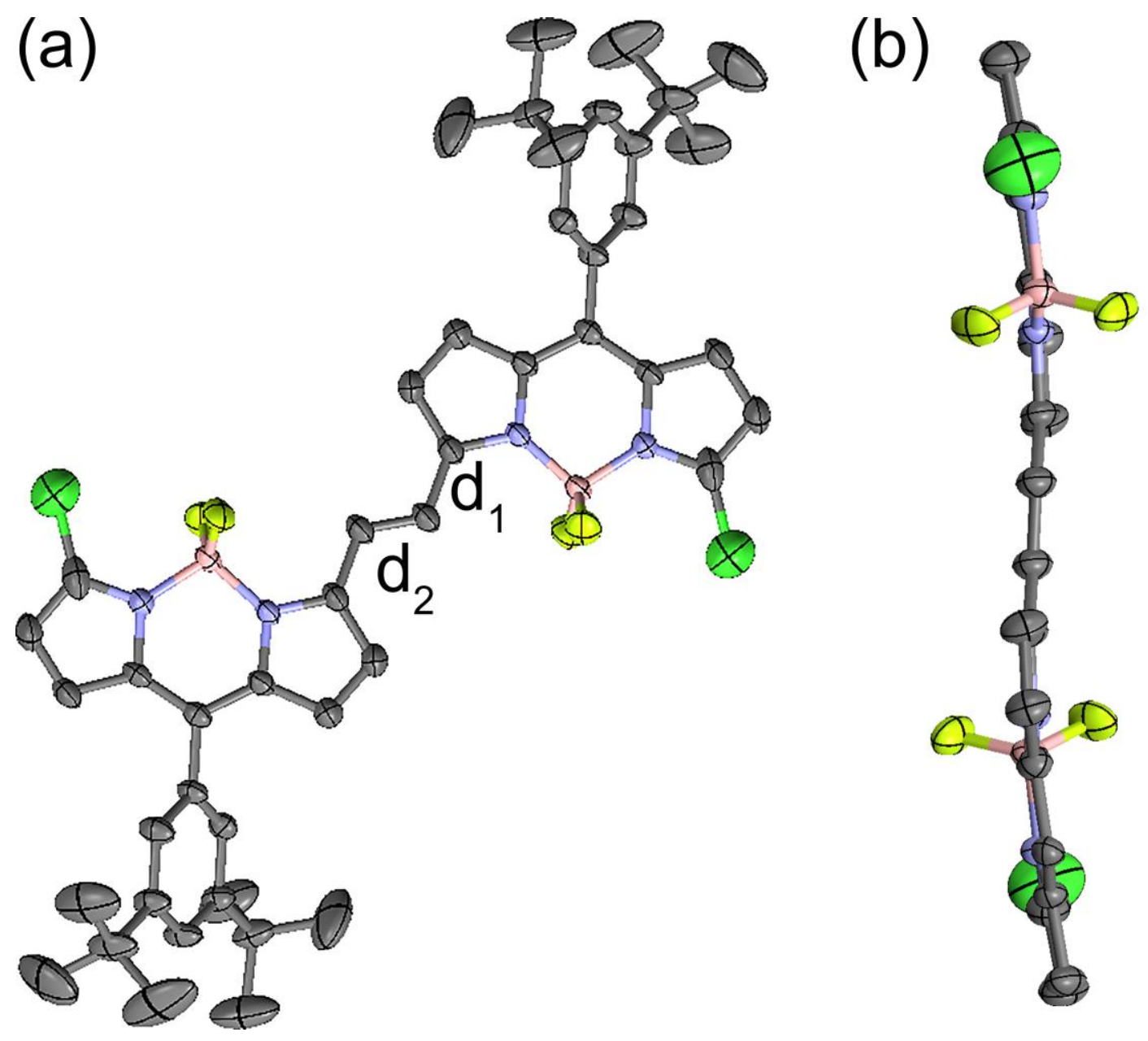

Figure S5. X-ray structure of dimer $\mathbf{2 \mathbf { C l } _ { 2 }}$ with thermal ellipsoids shown at the $50 \%$ level: (a) top and (b) side views. Hydrogen atoms are omitted for clarity. C, gray; N, blue; B, pink; F, light green; $\mathrm{Cl}$, green. 
Table S4. Crystal data and structure refinement for $\mathbf{2} \mathbf{C l}_{2}$.

\begin{tabular}{|c|c|}
\hline Identification code & $2 \mathrm{Cl}_{2}$ \\
\hline Empirical formula & $\mathrm{C}_{48} \mathrm{H}_{52} \mathrm{~B}_{2} \mathrm{Cl}_{2} \mathrm{~F}_{4} \mathrm{~N}_{4}$ \\
\hline Formula weight & 853.45 \\
\hline Temperature/K & 298.15 \\
\hline Crystal system & monoclinic \\
\hline Space group & $\mathrm{P} 2{ }_{1} / \mathrm{c}$ \\
\hline $\mathrm{a} / \AA ̊$ & $7.4479(9)$ \\
\hline $\mathrm{b} / \AA ̊$ & $16.707(2)$ \\
\hline $\mathrm{c} / \AA ̊$ & $18.802(2)$ \\
\hline$\alpha /{ }^{\circ}$ & 90 \\
\hline$\beta /{ }^{\circ}$ & $99.091(5)$ \\
\hline$\gamma /{ }^{\circ}$ & 90 \\
\hline Volume/Å3 & $2310.2(5)$ \\
\hline $\mathrm{Z}$ & 2 \\
\hline pcalcg/cm3 & 1.177 \\
\hline$\mu / \mathrm{mm} 1$ & 0.135 \\
\hline $\mathrm{F}(000)$ & 864.0 \\
\hline Crystal size/mm3 & $0.22 \times 0.21 \times 0.2$ \\
\hline Radiation & $\operatorname{MoK} \alpha(\lambda=0.71073)$ \\
\hline \multicolumn{2}{|c|}{$2 \Theta$ range for data collection $/{ }^{\circ} 5.54$ to 55.346} \\
\hline Index ranges & $-9 \leq \mathrm{h} \leq 9,-21 \leq \mathrm{k} \leq 21,-24 \leq 1 \leq 24$ \\
\hline Reflections collected & 28805 \\
\hline Independent reflections & $5356[$ Rint $=0.0502$, Rsigma $=0.0339]$ \\
\hline Data/restraints/parameters & $5356 / 630 / 277$ \\
\hline Goodness-of-fit on F2 & 1.162 \\
\hline Final $\mathrm{R}$ indexes $[\mathrm{I}>=2 \sigma(\mathrm{I})]$ & $\mathrm{R} 1=0.0924, \mathrm{wR} 2=0.2619$ \\
\hline Final $\mathrm{R}$ indexes [all data] & $\mathrm{R} 1=0.1229, \mathrm{wR} 2=0.2851$ \\
\hline
\end{tabular}




\section{Photophysical data}
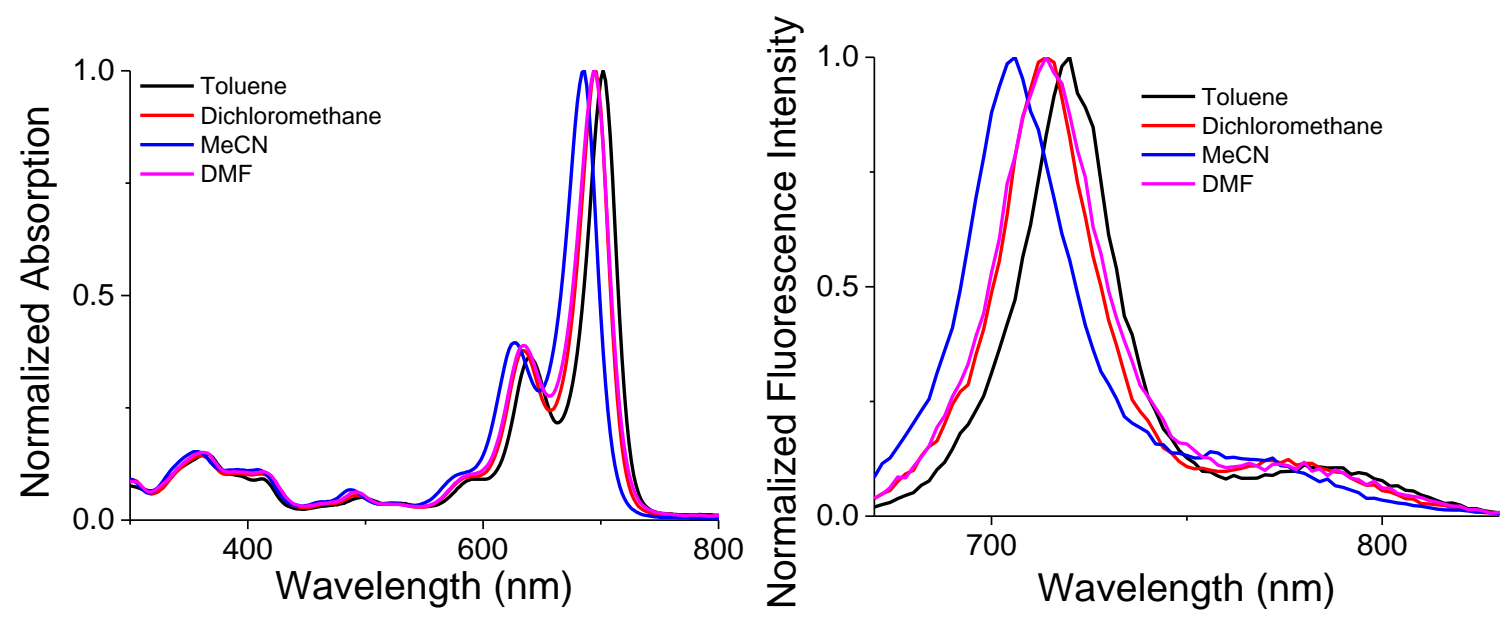

Figure S6. Overlaid normalized absorption and fluorescence emission spectra of $\mathbf{2}$ in different solvents at room temperature.

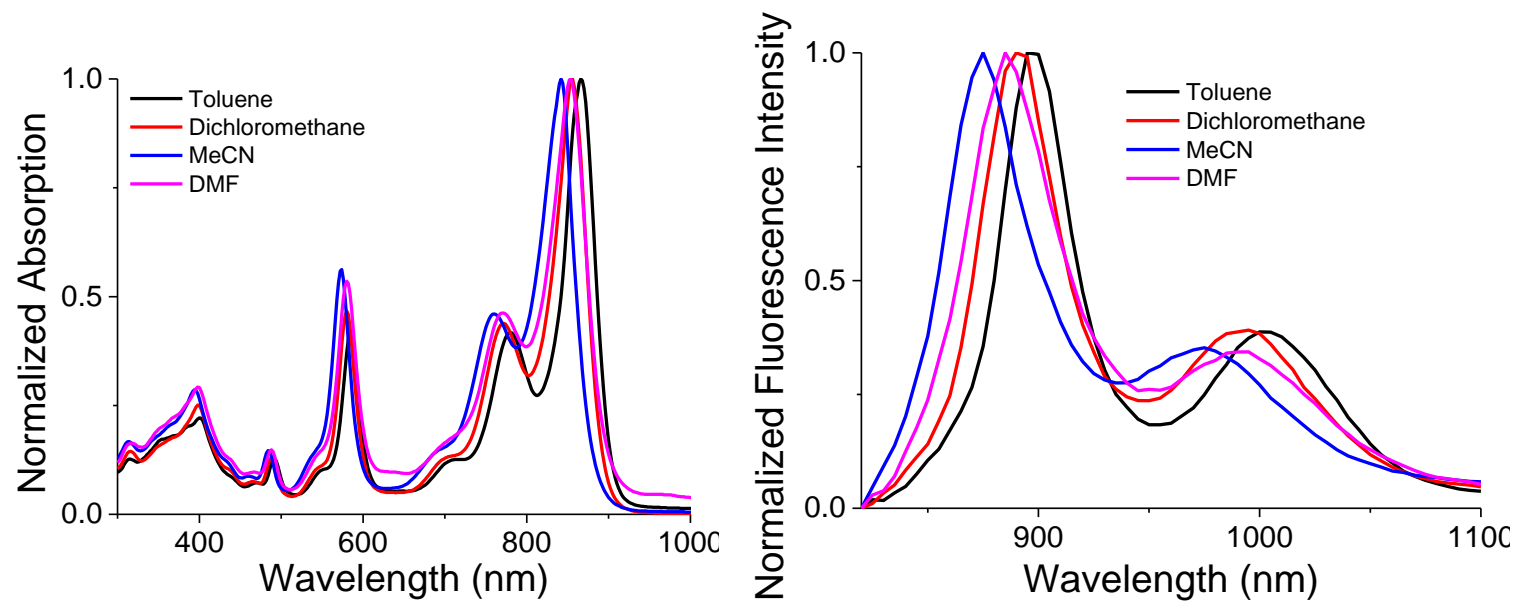

Figure S7. Overlaid normalized absorption and fluorescence emission spectra of $\mathbf{3}$ in different solvents at room temperature. 

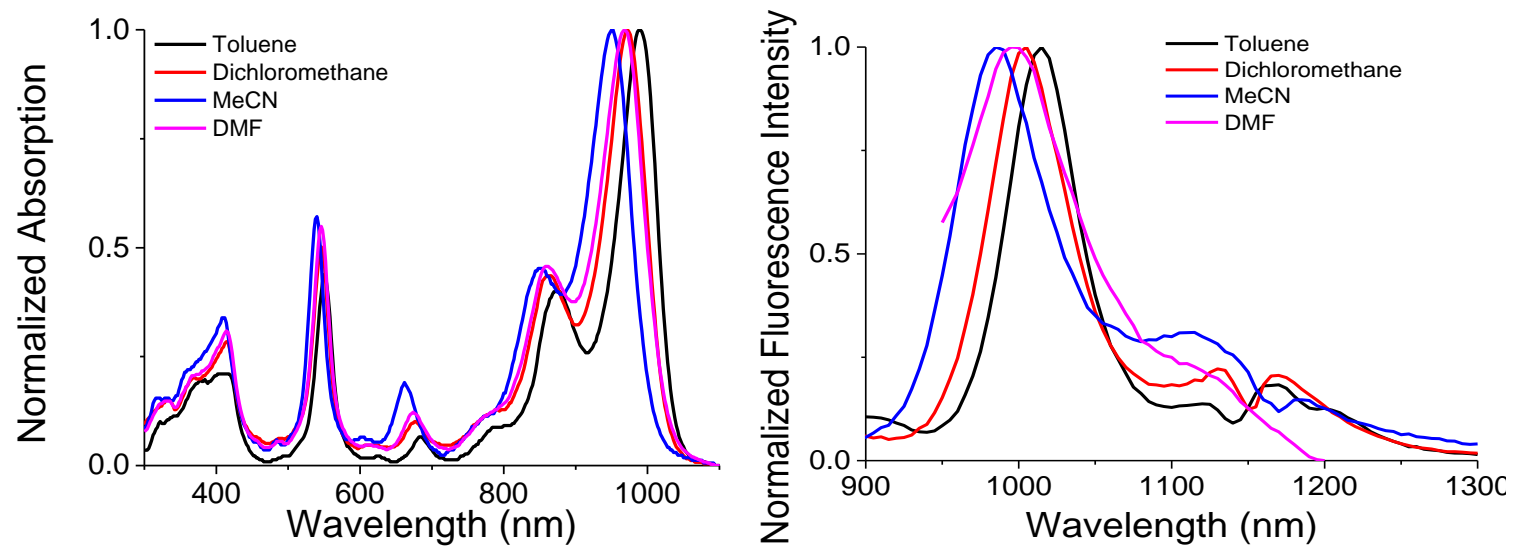

Figure S8. Overlaid normalized absorption and fluorescence emission spectra of $\mathbf{4}$ in different solvents at room temperature.
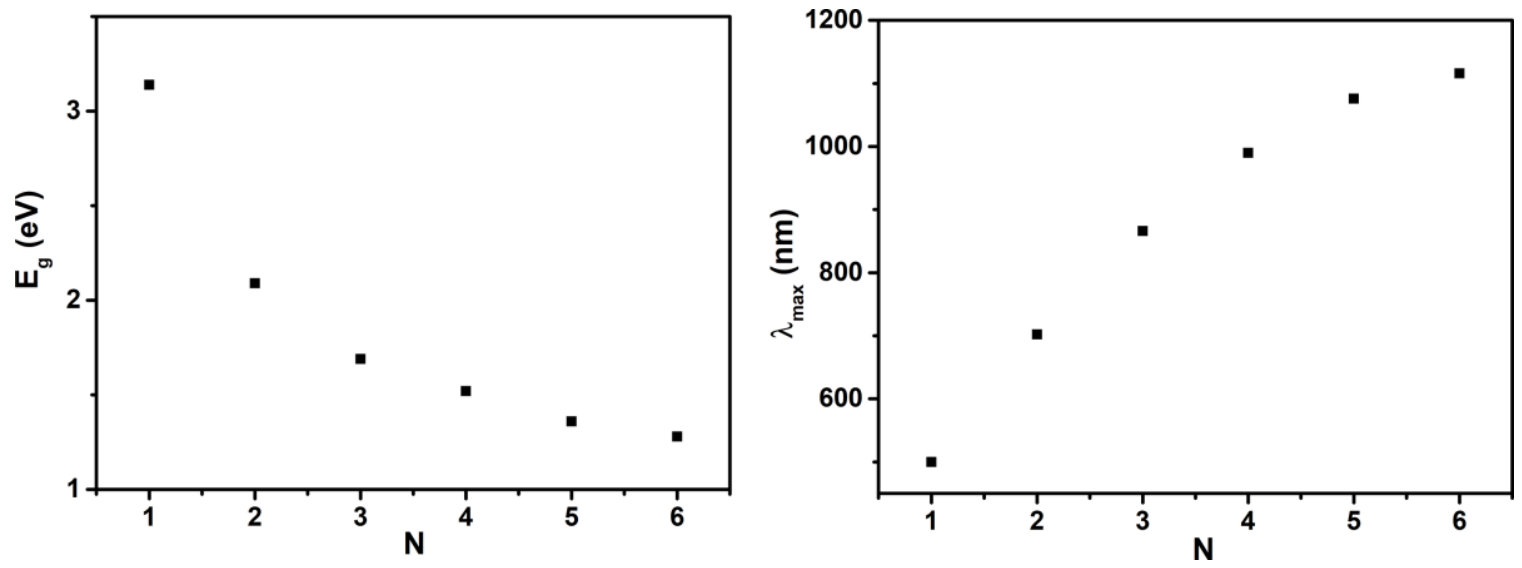

Figure S9 Plot of band gaps Eg (left) and maxima absorption peaks (right) of monomer 1 and arrays 2-6 in toluene versus the numbers of BODIPY units $(\mathrm{N})$. The Eg was obtained from the DFT calculated HOMO and LUMO energy levels. 


\section{Photothermal studies}
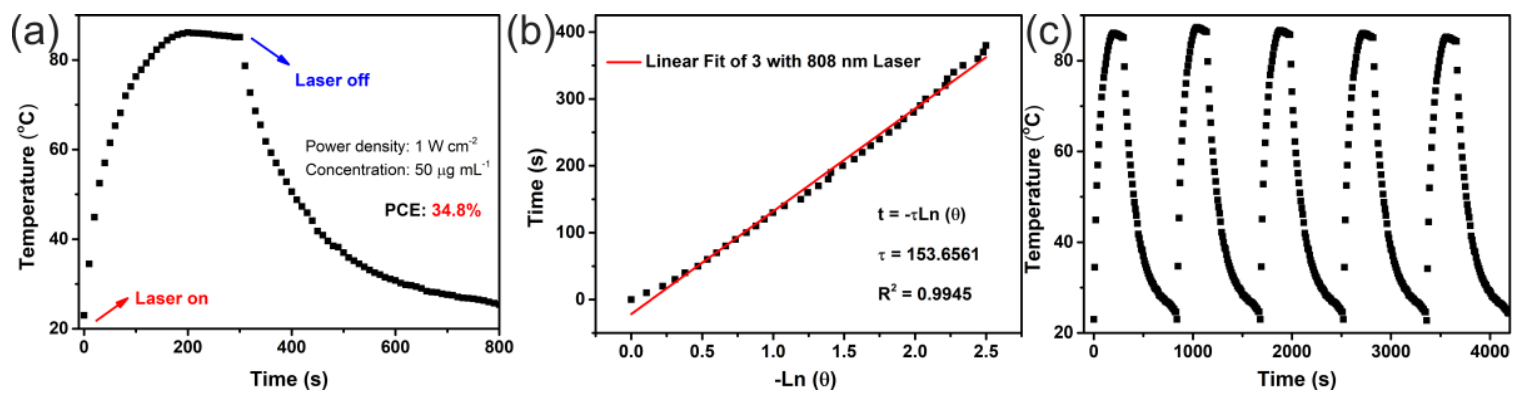

Figure S10. (a) Photothermal effect of $50 \mu \mathrm{g} / \mathrm{mL}$ oligomer 3 upon $808 \mathrm{~nm}$ laser irradiation $\left(1 \mathrm{~W} \mathrm{~cm}^{-2}\right)$ in toluene which was turned off after irradiation for 9 minutes and (b) plot of cooling time vs. negative natural logarithm of the temperature change obtained from the cooling stage. (c) Temperature evolutions of $50 \mu \mathrm{g} / \mathrm{mL}$ oligomer 3 under $808 \mathrm{~nm}$ laser irradiation $\left(1 \mathrm{~W} \mathrm{~cm}^{-2}\right)$ for 5 laser on/off cycles in toluene.

(a)

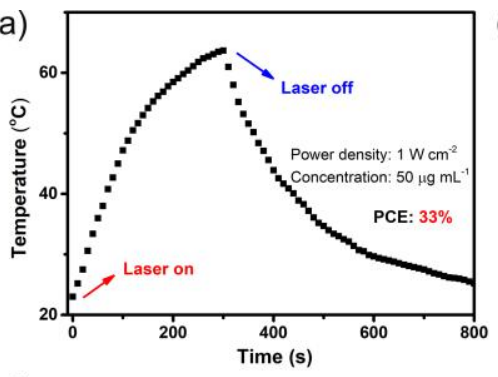

(d)

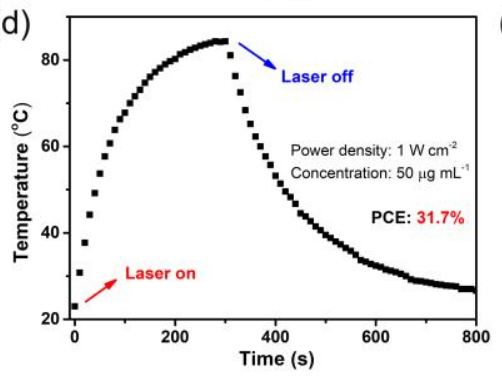

(b)
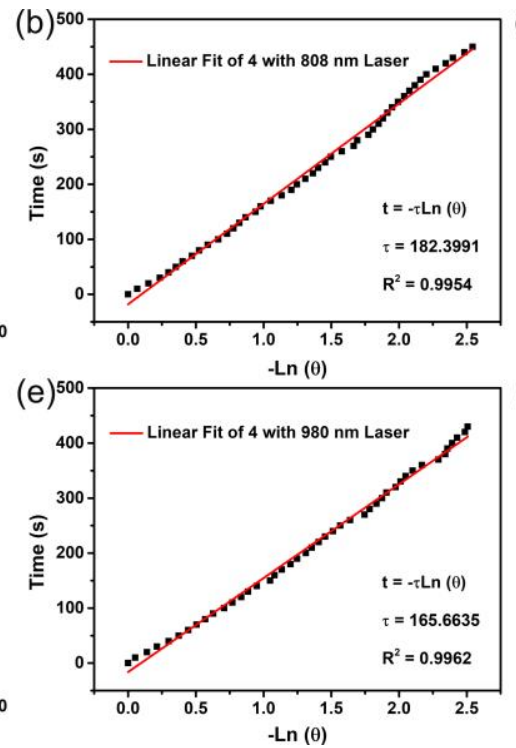

(c)

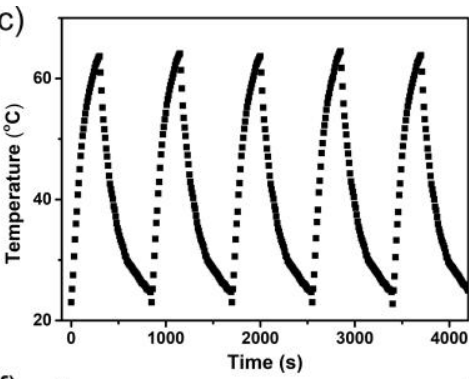

(f)

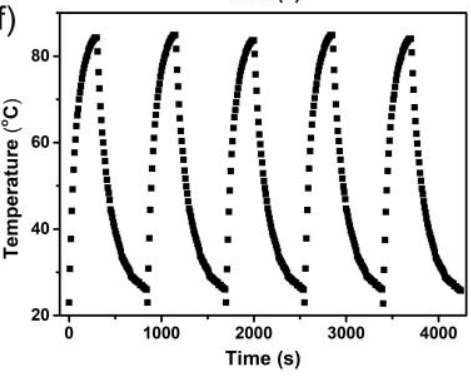

Figure S11. Photothermal effect of $50 \mu \mathrm{g} / \mathrm{mL}$ oligomer 4 upon $808 \mathbf{~ n m}$ (a-b) and $980 \mathbf{~ n m}$ (d-e) laser irradiation $\left(1 \mathrm{~W} \mathrm{~cm}^{-2}\right)$ in toluene which was turned off after irradiation for 9 minutes and plot of cooling time vs. negative natural logarithm of the temperature change obtained from the cooling stage.

(c) Temperature evolutions of $50 \mu \mathrm{g} / \mathrm{mL}$ oligomer 4 under $808 \mathbf{~ n m}$ (c) and $980 \mathrm{~nm}$ (f) laser irradiation $\left(1 \mathrm{~W} \mathrm{~cm}^{-2}\right)$ for 5 laser on/off cycles in toluene. 

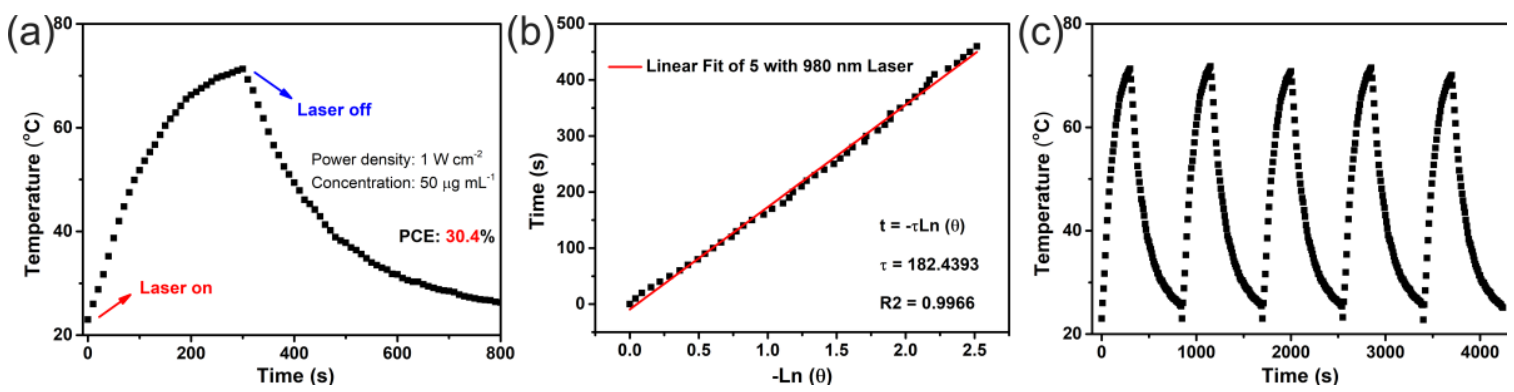

Figure S12. (a) Photothermal effect of $50 \mu \mathrm{g} / \mathrm{mL}$ oligomer 5 upon $980 \mathrm{~nm}$ laser irradiation $\left(1 \mathrm{~W} \mathrm{~cm}^{-2}\right)$ in toluene which was turned off after irradiation for 9 minutes and (b) plot of cooling time vs. negative natural logarithm of the temperature change obtained from the cooling stage. (c) Temperature evolutions of $50 \mu \mathrm{g} / \mathrm{mL}$ oligomer 5 under $980 \mathrm{~nm}$ laser irradiation $\left(1 \mathrm{~W} \mathrm{~cm}^{-2}\right)$ for 5 laser on/off cycles in toluene.
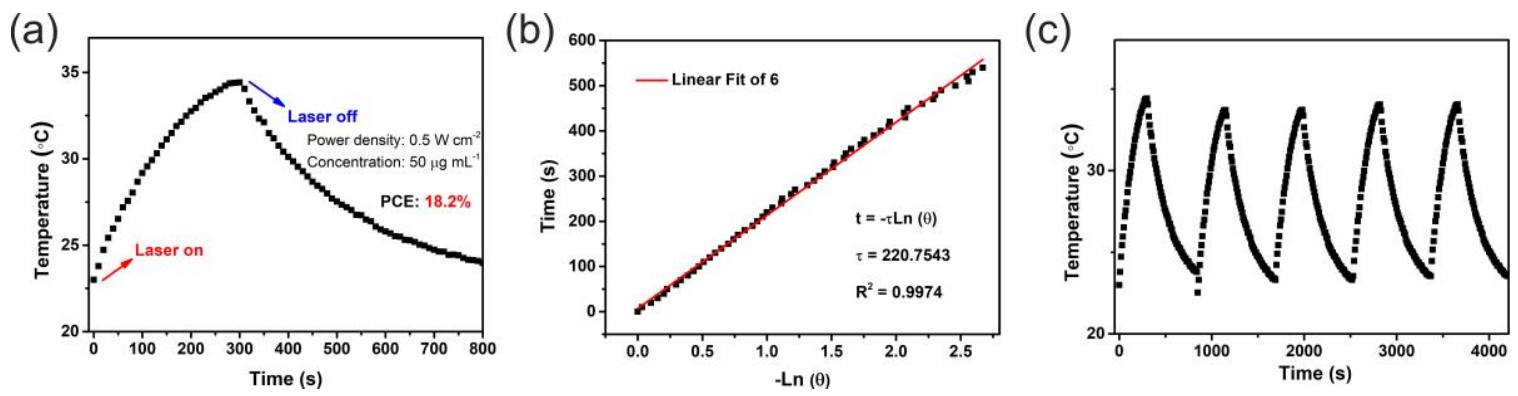

Figure S13. (a) Photothermal effect of $50 \mu \mathrm{g} / \mathrm{mL}$ oligomer 6 upon $980 \mathrm{~nm}$ laser irradiation $\left(1 \mathrm{~W} \mathrm{~cm}^{-2}\right)$ in toluene which was turned off after irradiation for 9 minutes and (b) plot of cooling time vs. negative natural logarithm of the temperature change obtained from the cooling stage. (c) Temperature evolutions of $50 \mu \mathrm{g} / \mathrm{mL}$ oligomer 6 under $980 \mathrm{~nm}$ laser irradiation $\left(1 \mathrm{~W} \mathrm{~cm}^{-2}\right)$ for 5 laser on/off cycles in toluene.

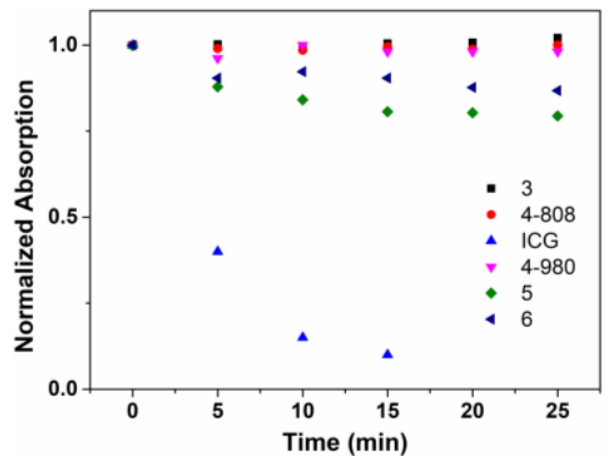

Figure S14. Comparison of the photostability of 3, 4, ICG, 5 and 6 in toluene under continuous irradiation with corresponding laser ( $808 \mathrm{~nm}$ for $\mathbf{3 , 4}$ and ICG, $980 \mathrm{~nm}$ for $\mathbf{4 , 5}$ and $\mathbf{6}$ ) at a power of 1 $\mathrm{W} \mathrm{cm}{ }^{-2}$. 


\section{Characterization and Properties of Nanoparticles.}

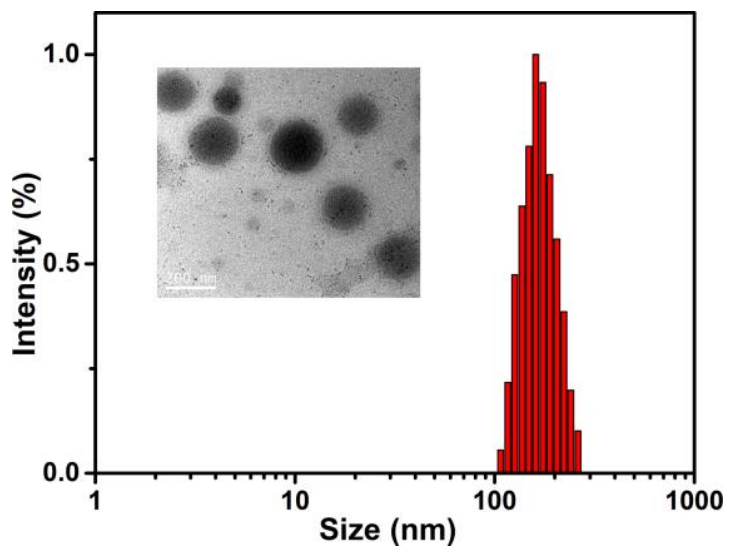

Figure S15. DLS size distribution of 3-NPs and its TEM images. The scale bar is $200 \mathrm{~nm}$.
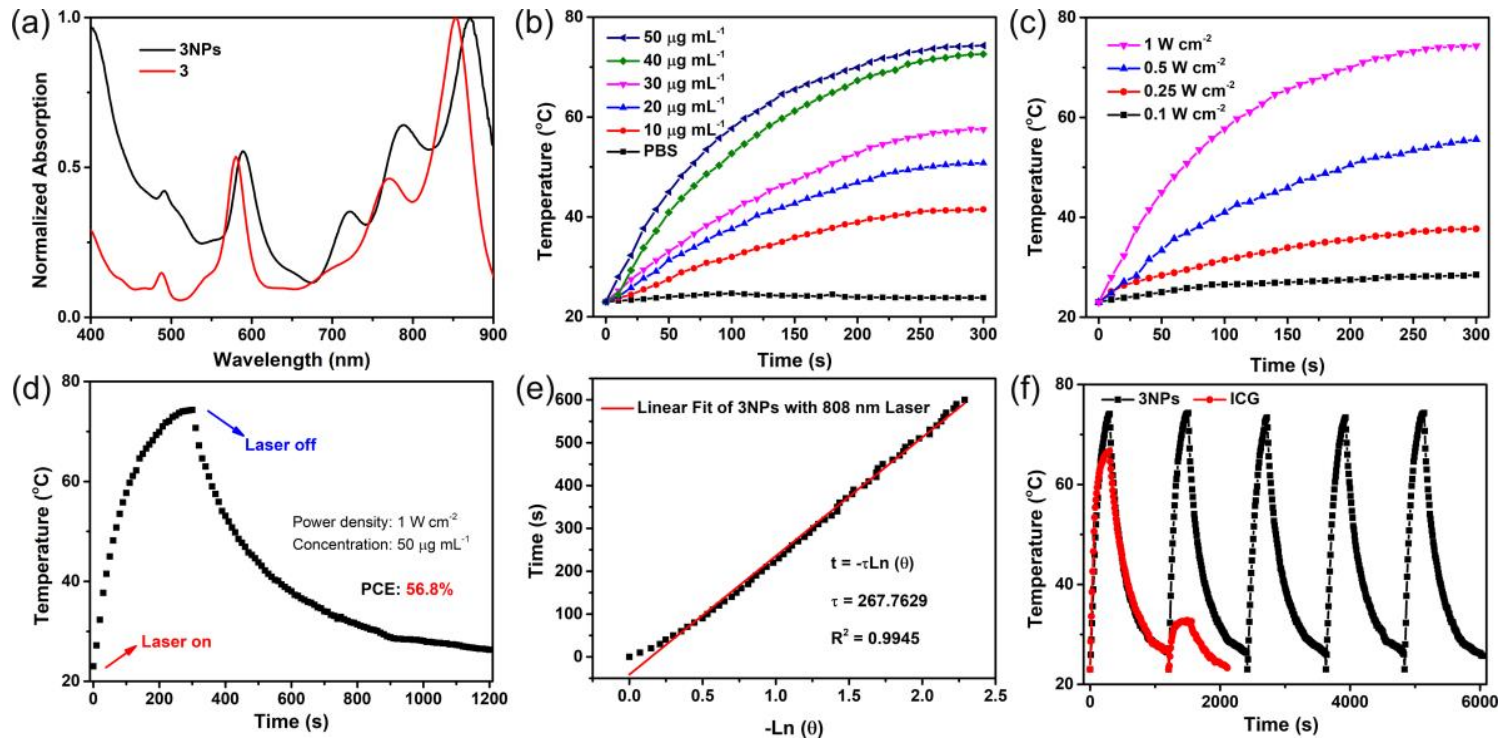

Figure S16. (a) Normalized absorption spectra of trimer 3 in DMF and 3NPs in water, respectively. (b) Photothermal conversion of 3NPs at different concentrations $(0-50 \mu \mathrm{g} \mathrm{mL}-1)$ under $808 \mathrm{~nm}$ laser irradiation $\left(1 \mathrm{~W} \mathrm{~cm}^{-2}\right)$. (c) Photothermal conversion of 3NPs $(50 \mu \mathrm{g} \mathrm{mL}-1)$ under $808 \mathrm{~nm}$ laser irradiation with different exposure intensity (0.1-1.0 $\mathrm{W} \mathrm{cm}^{-2}$ ). (d) Photothermal effect of 3NPs upon laser irradiation $\left(1 \mathrm{~W} \mathrm{~cm}^{-2}\right)$ in water which was turned off after irradiation for 15 minutes and (e) plot of cooling time vs. negative natural logarithm of the temperature change obtained from the cooling stage. (f) Temperature evolutions of the 3NPs and ICG under laser irradiation $\left(1 \mathrm{~W} \mathrm{~cm}^{-2}\right)$ for 5 laser on/off cycles 

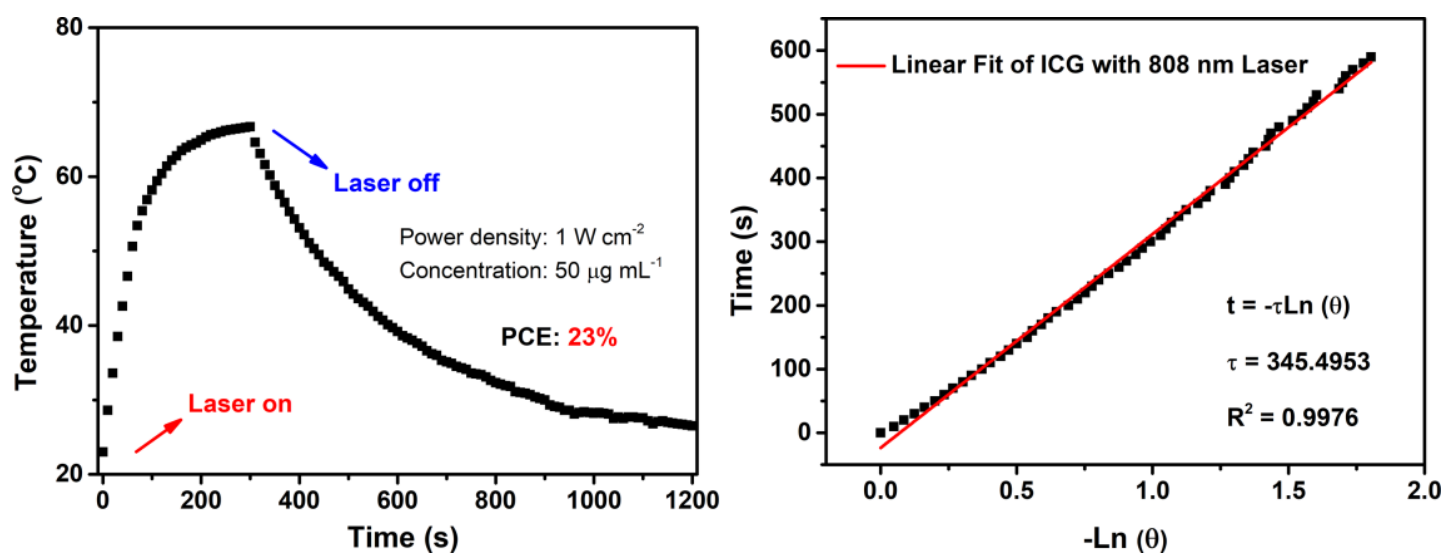

Figure S17. Photothermal effect of ICG upon laser irradiation $\left(1 \mathrm{~W} \mathrm{~cm}^{-2}\right)$ in water which was turned off after irradiation for 15 minutes and plot of cooling time vs. negative natural logarithm of the temperature change obtained from the cooling stage. 
8. ${ }^{1} \mathrm{H}$ NMR, ${ }^{13} \mathrm{C}$ NMR and HRMS for all new compounds

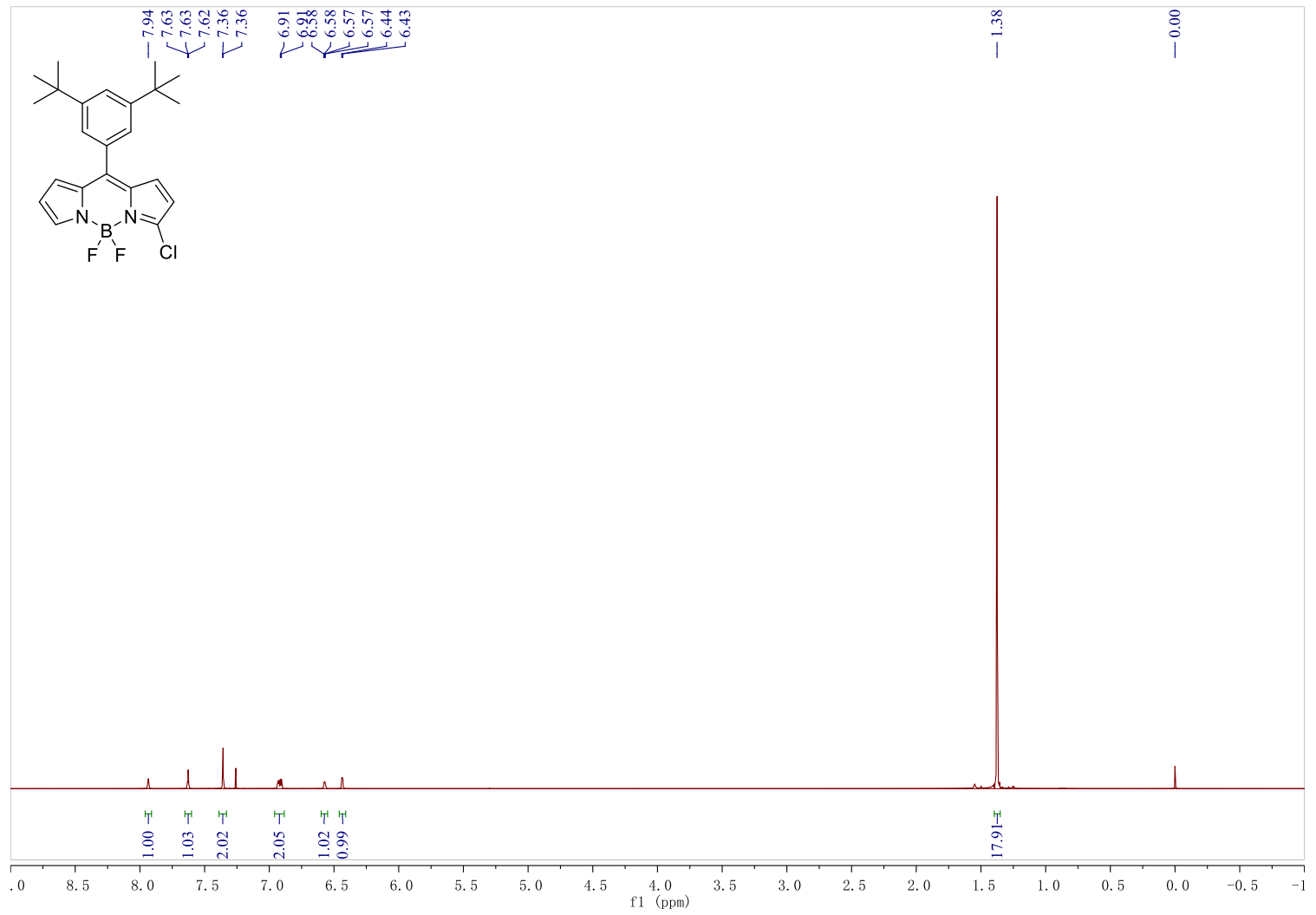

${ }^{1} \mathrm{H}$ NMR (500 MHz) spectrum of $\mathbf{1 C l}$ in $\mathrm{CDCl}_{3}$.

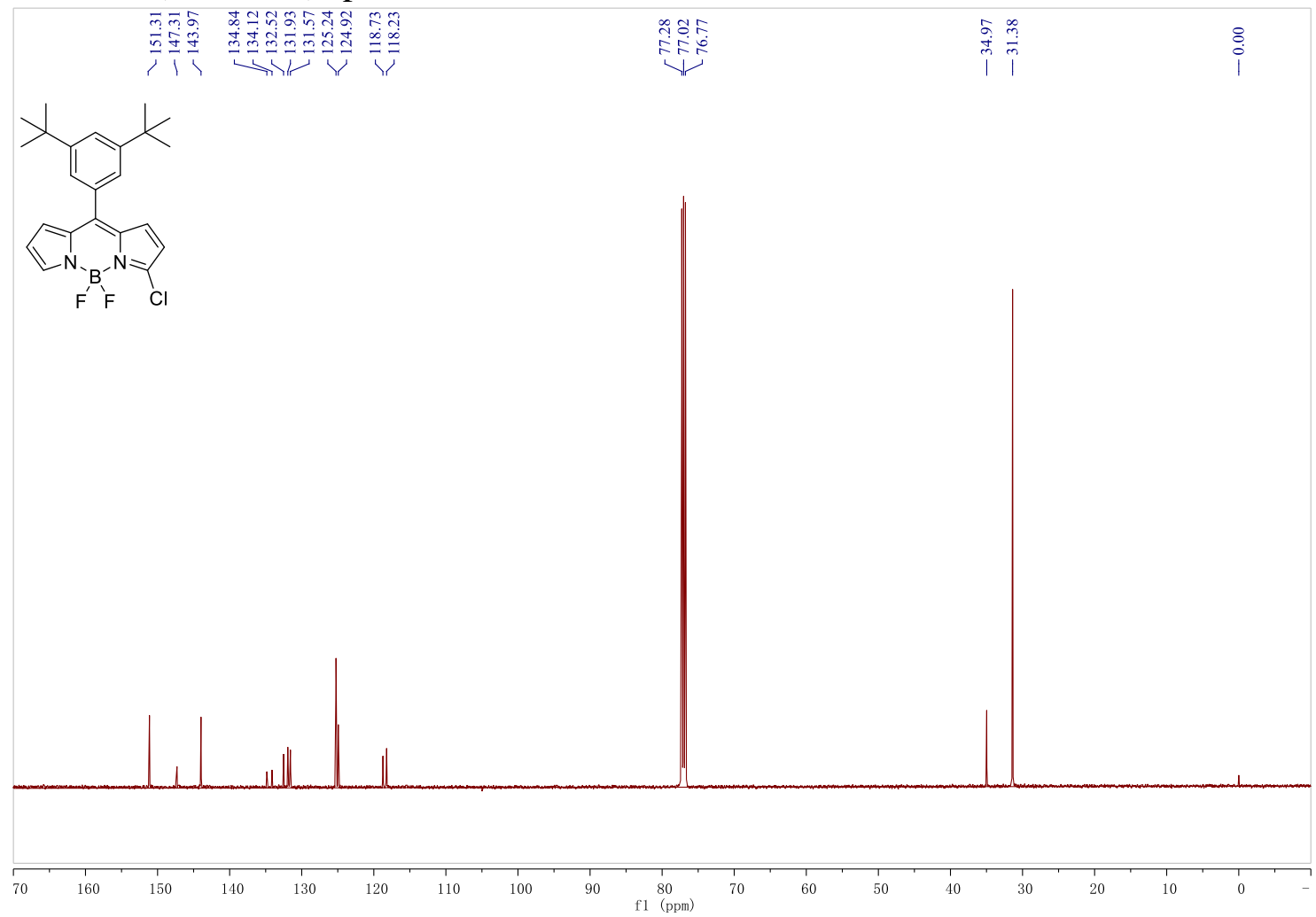


${ }^{13} \mathrm{C}$ NMR (500 MHz) spectrum of $\mathbf{1 C l}$ in $\mathrm{CDCl}_{3}$.

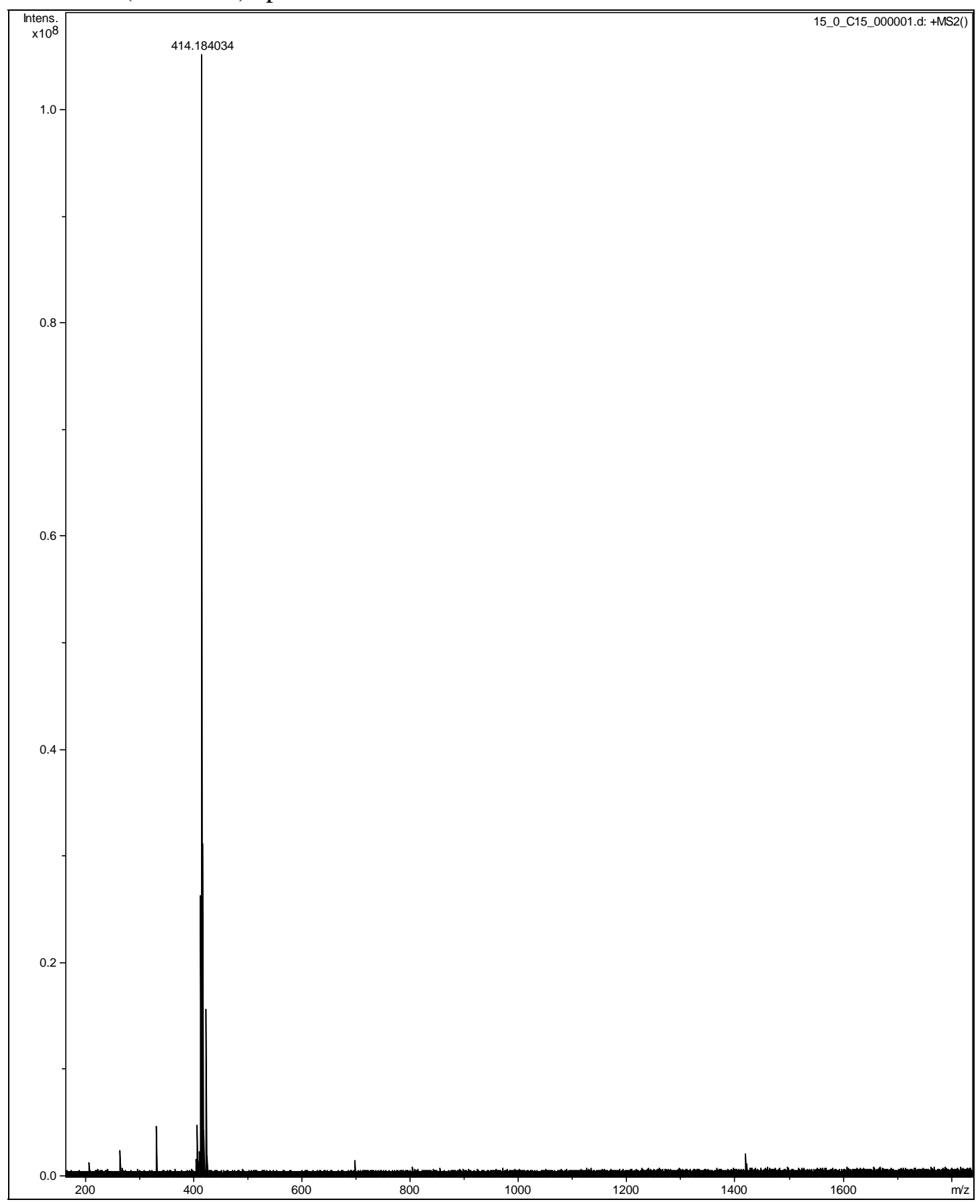

HRMS for $\mathbf{1 C l}$. 


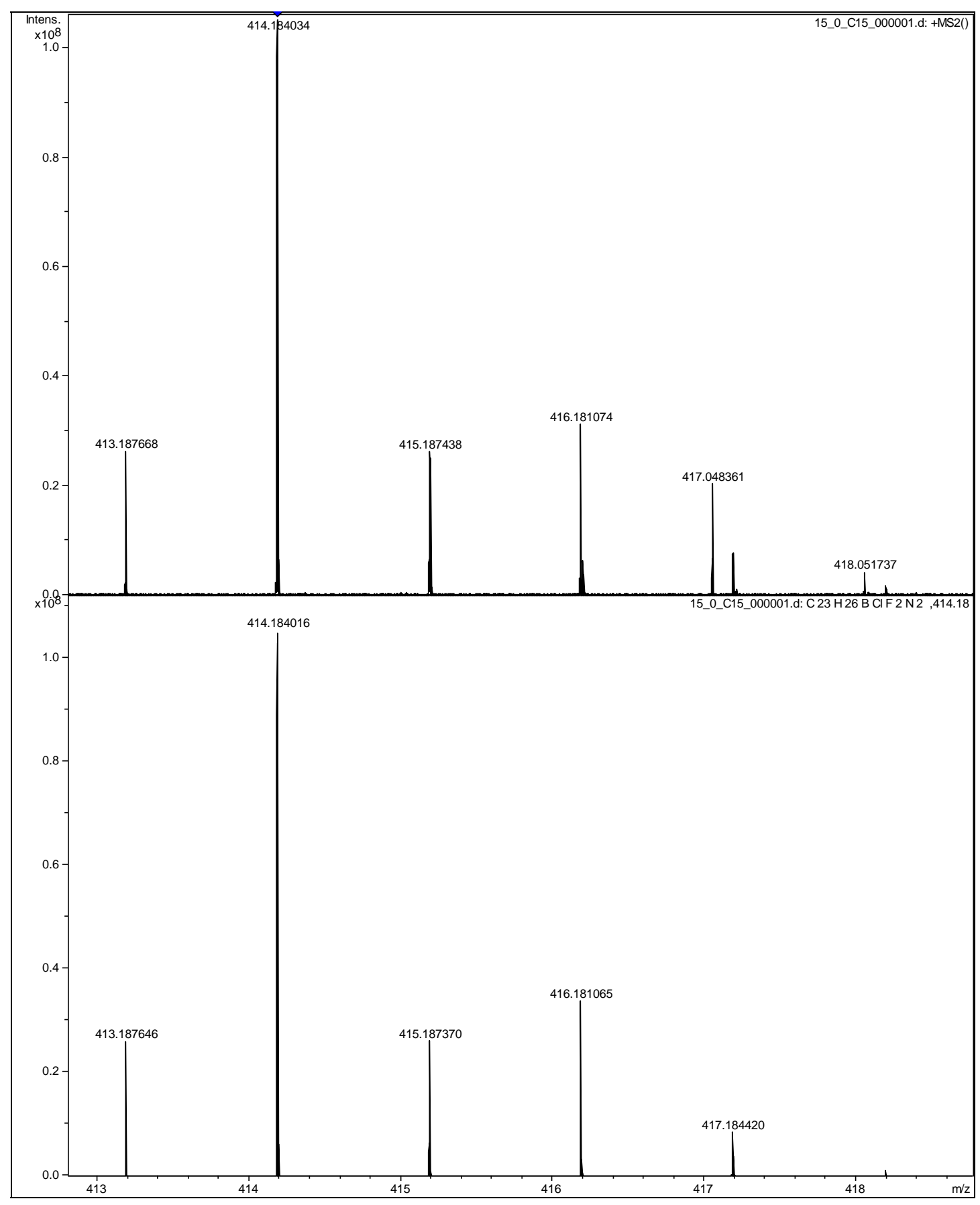

Observed (top) and calculated (bottom) HRMS for $\mathbf{1 C l}$. 


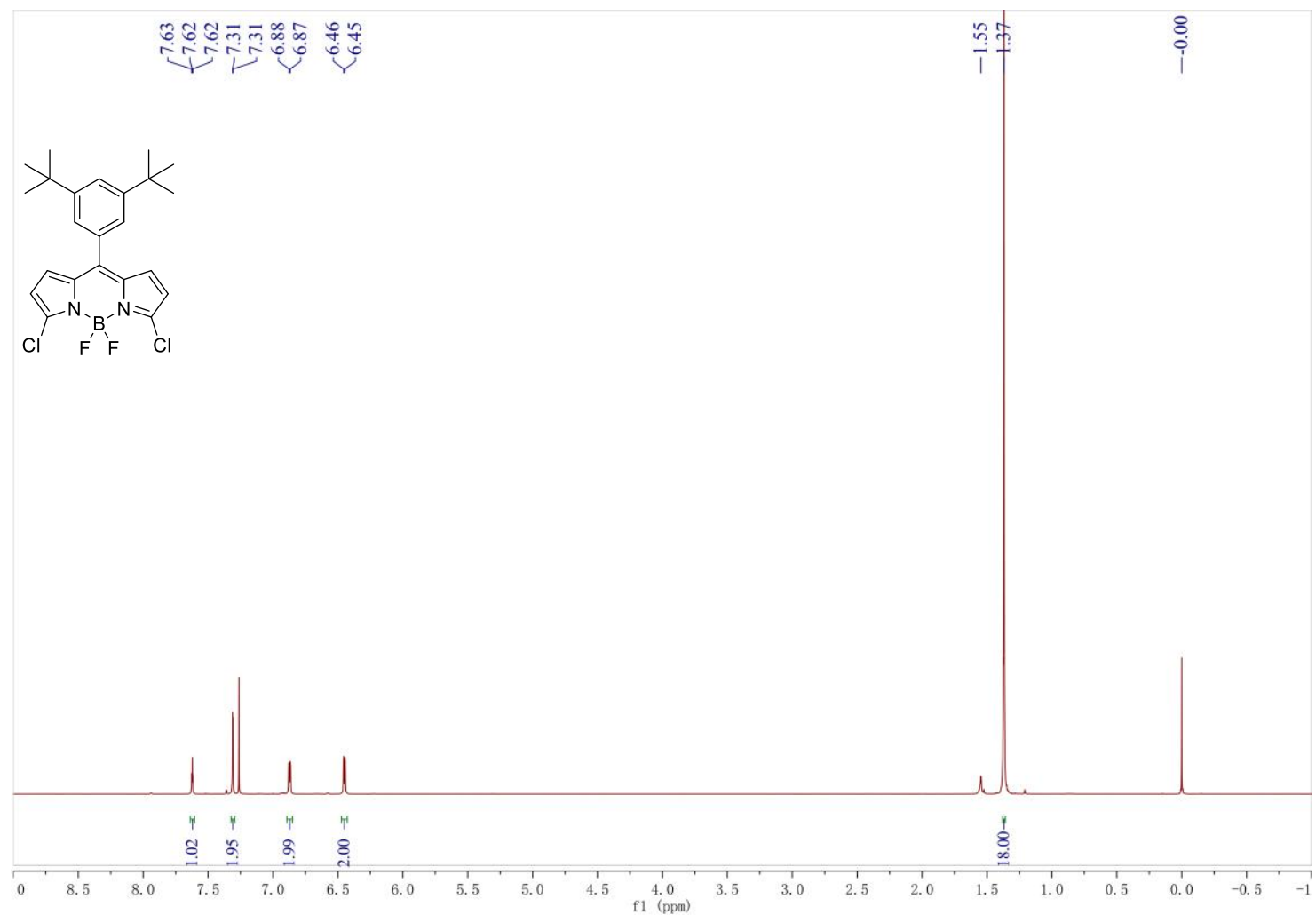

${ }^{1} \mathrm{H}$ NMR (400 MHz) spectrum of $\mathbf{1} \mathbf{C l}_{2}$ in $\mathrm{CDCl}_{3}$.
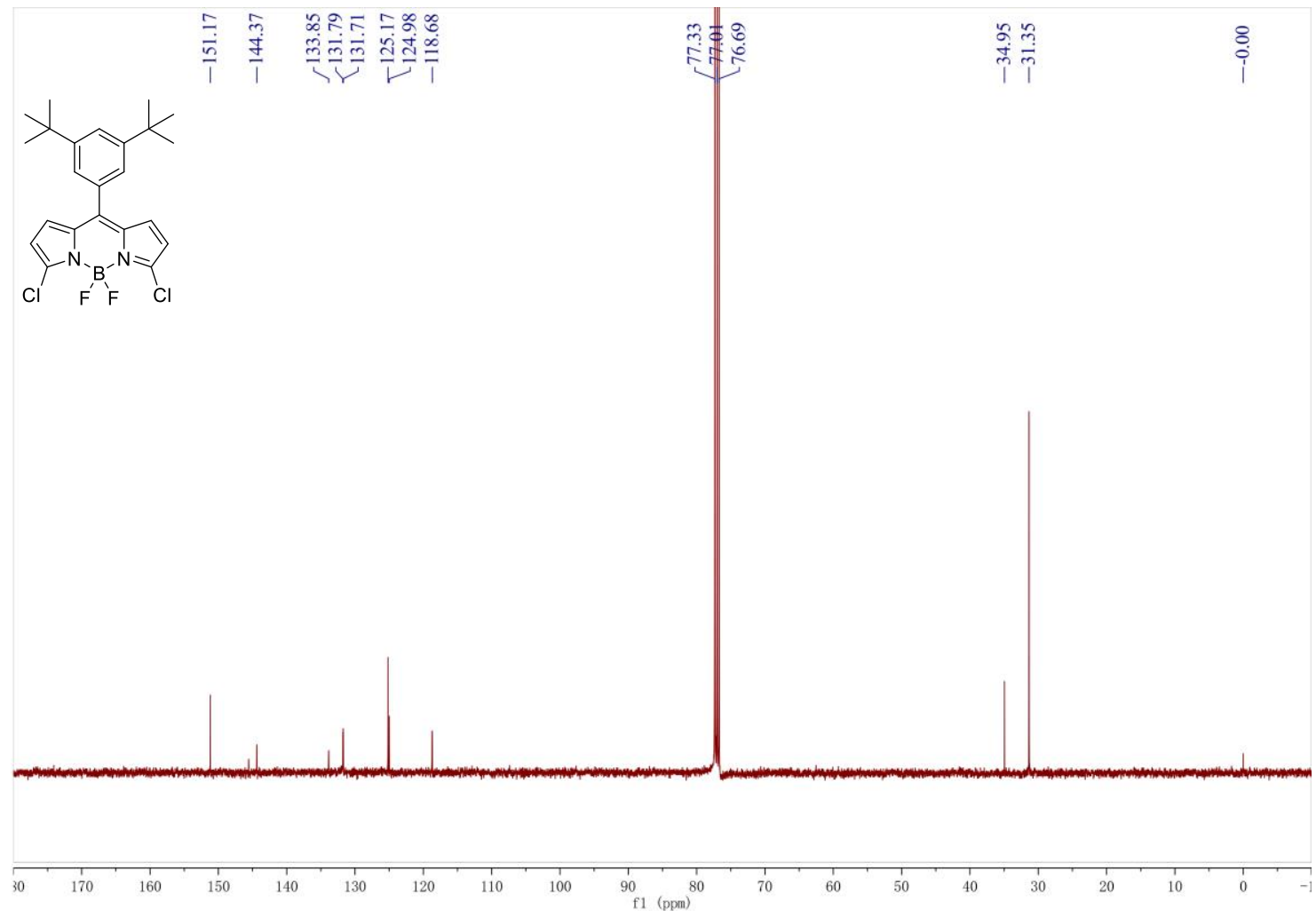

${ }^{13} \mathrm{C} \mathrm{NMR}(400 \mathrm{MHz})$ spectrum of $\mathbf{1 C l} \mathbf{l}_{2}$ in $\mathrm{CDCl}_{3}$. 


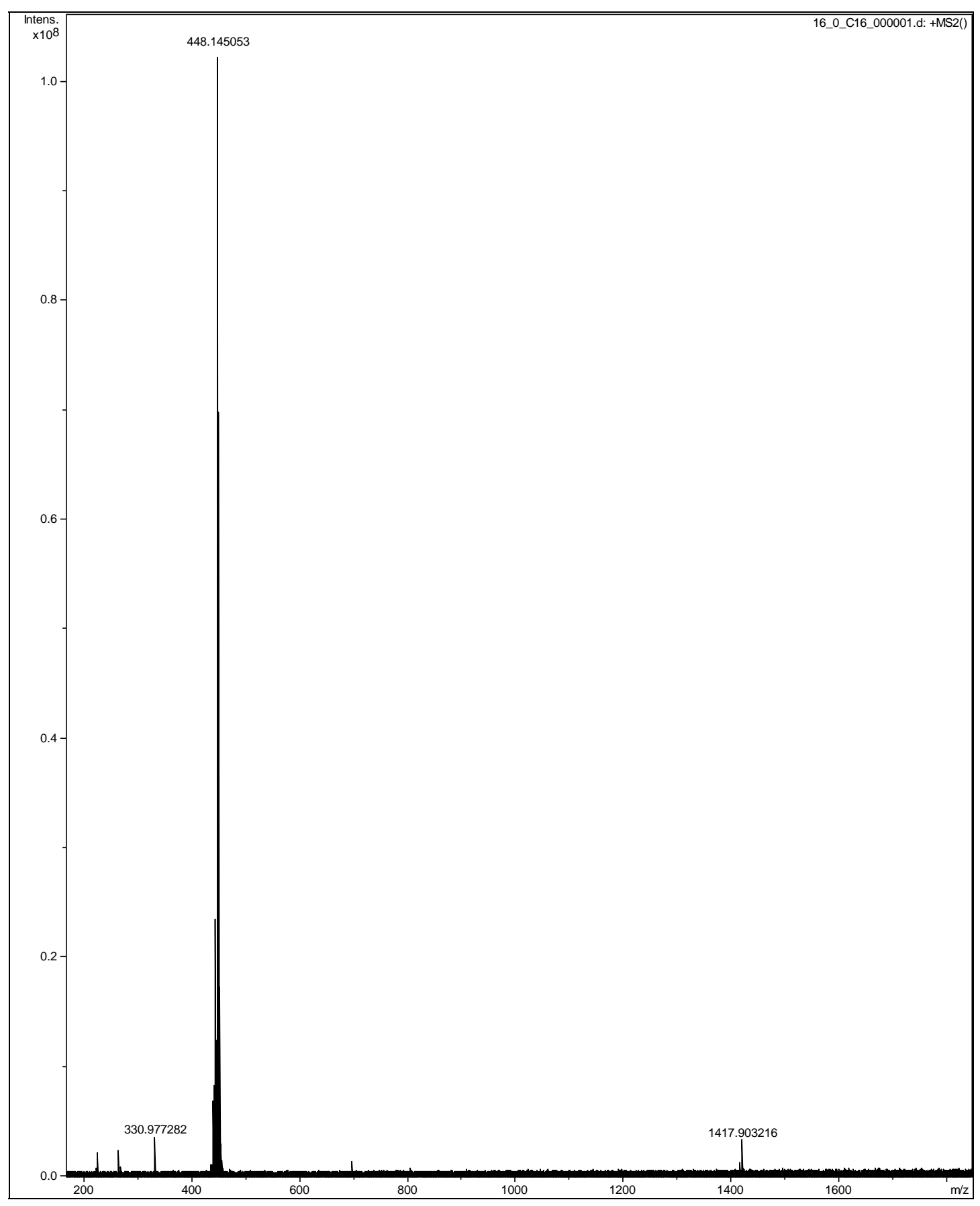

HRMS for $\mathbf{1 C l} 2$. 


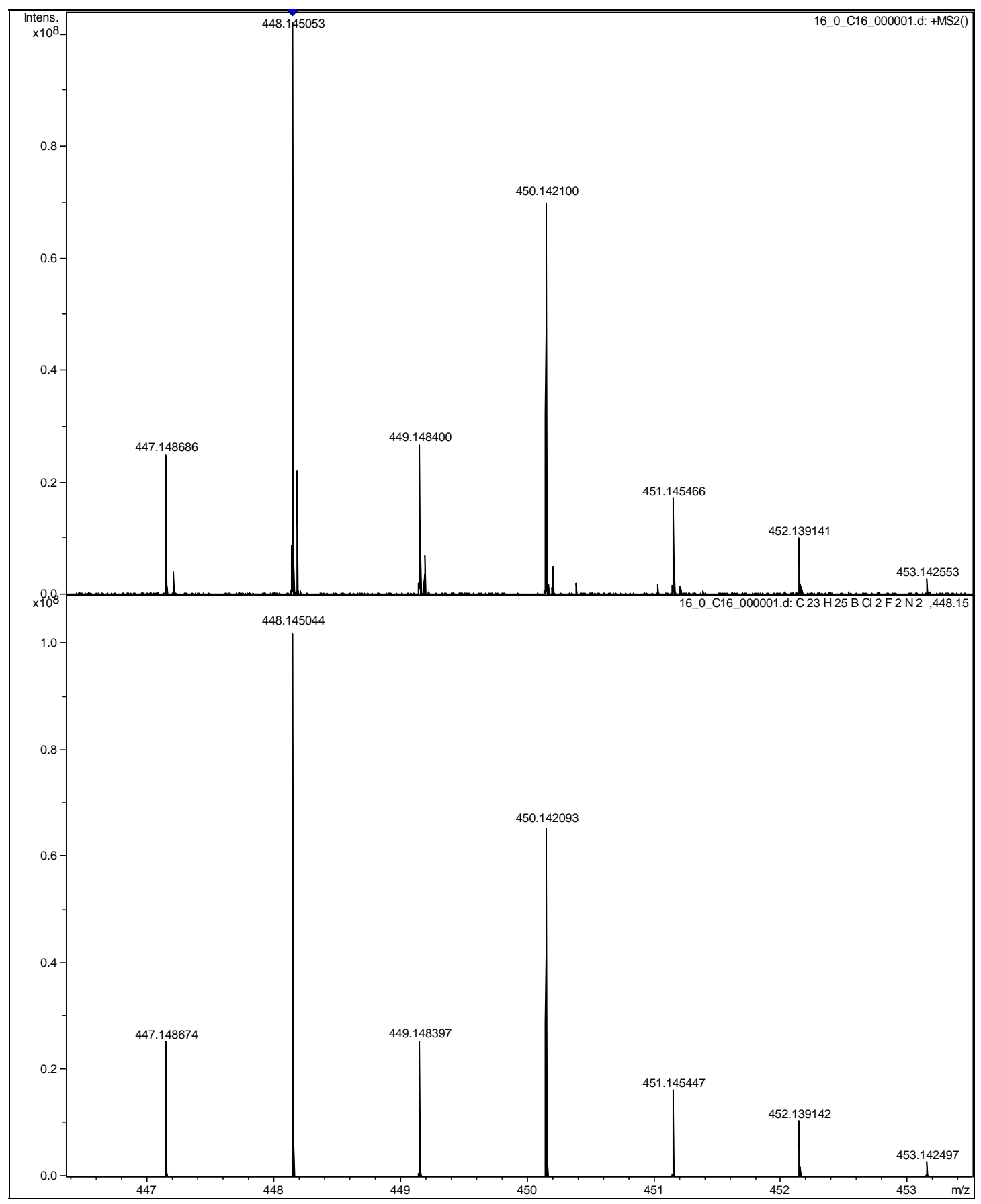

Observed (top) and calculated (bottom) HRMS for $\mathbf{1 C l}$. 


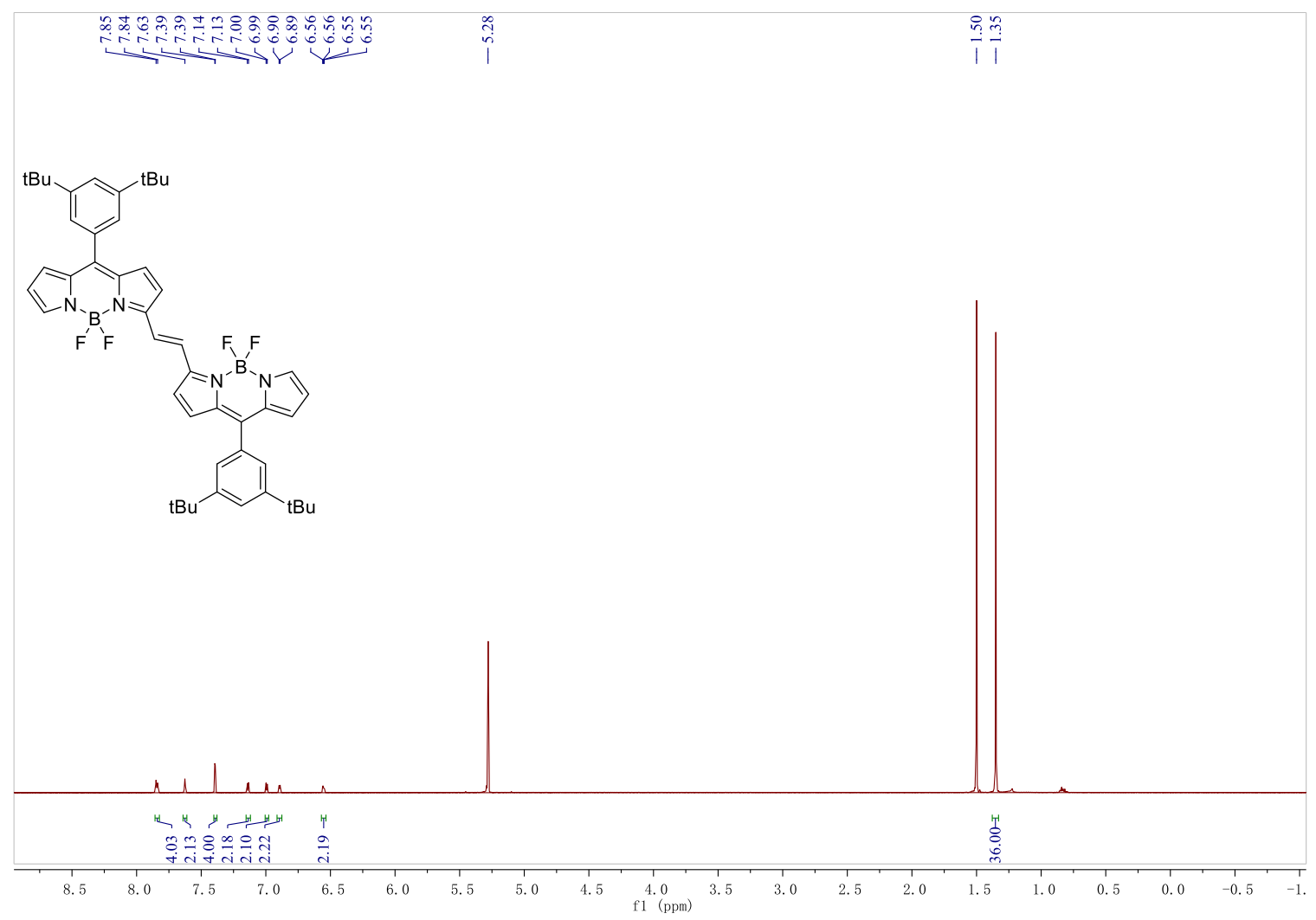

${ }^{1} \mathrm{H}$ NMR (500 MHz) spectrum of 2 in $\mathrm{CD}_{2} \mathrm{Cl}_{2}$.

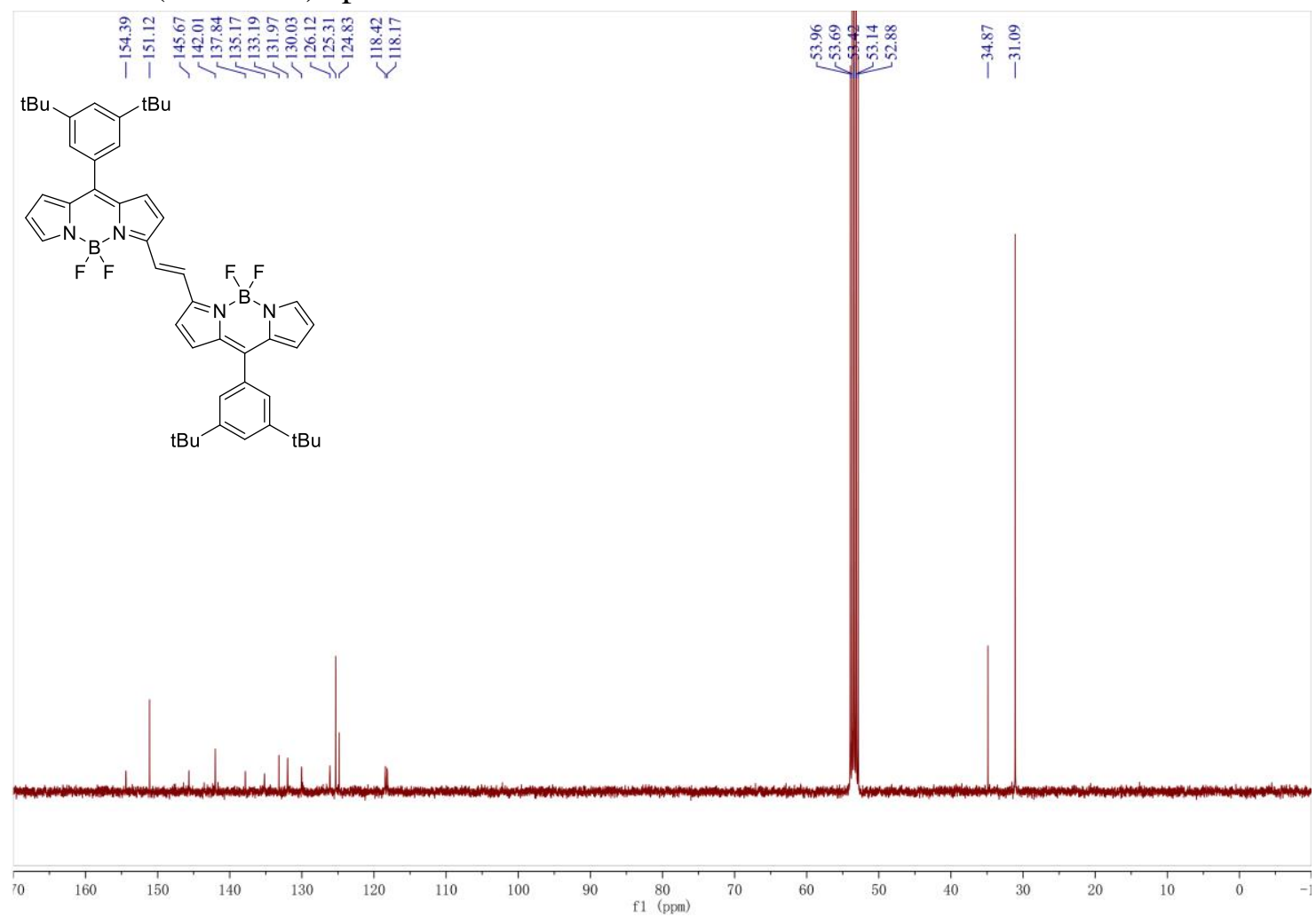

${ }^{13} \mathrm{C}$ NMR (400 MHz) spectrum of 2 in $\mathrm{CD}_{2} \mathrm{Cl}_{2}$. 


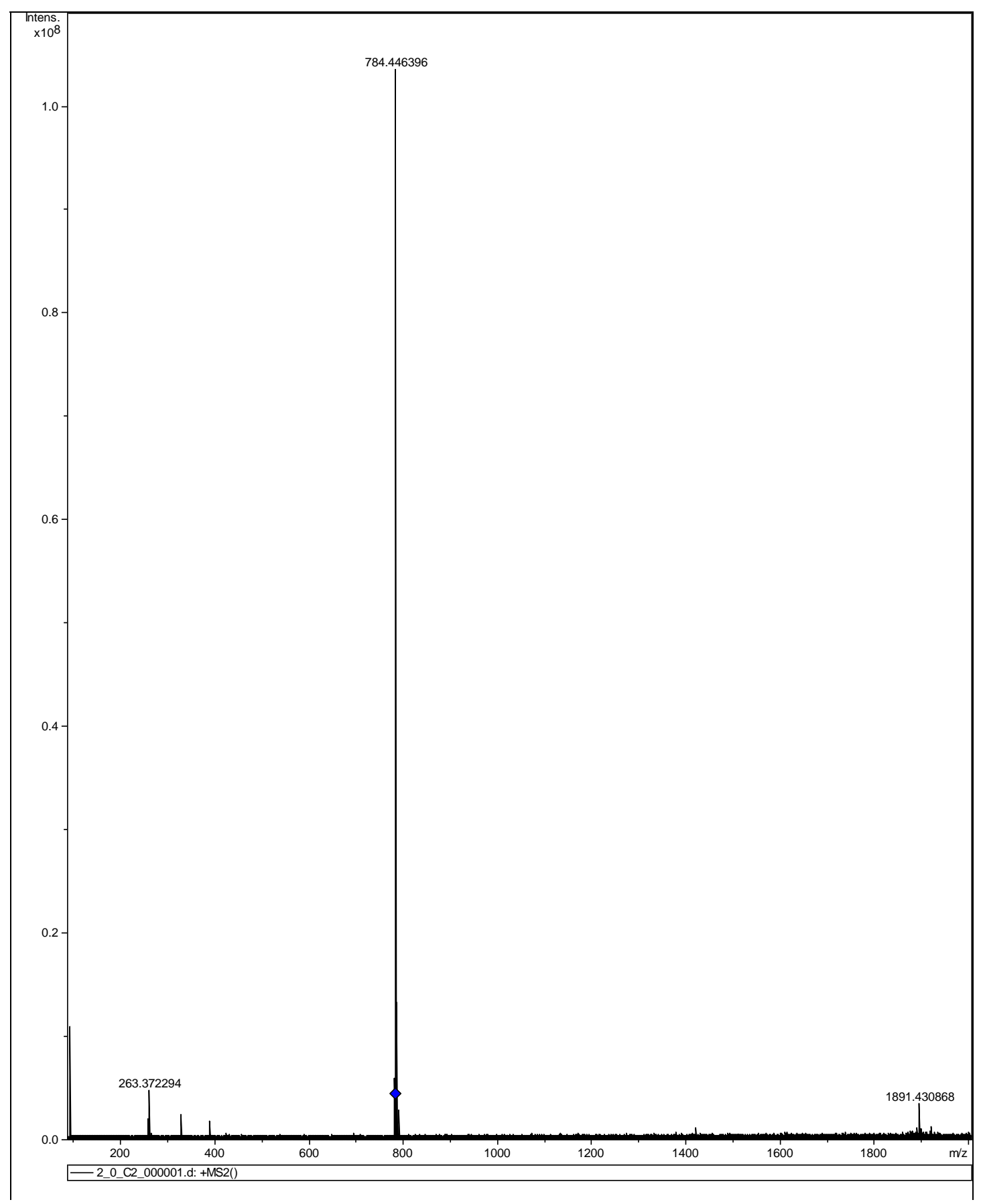

HRMS for 2. 


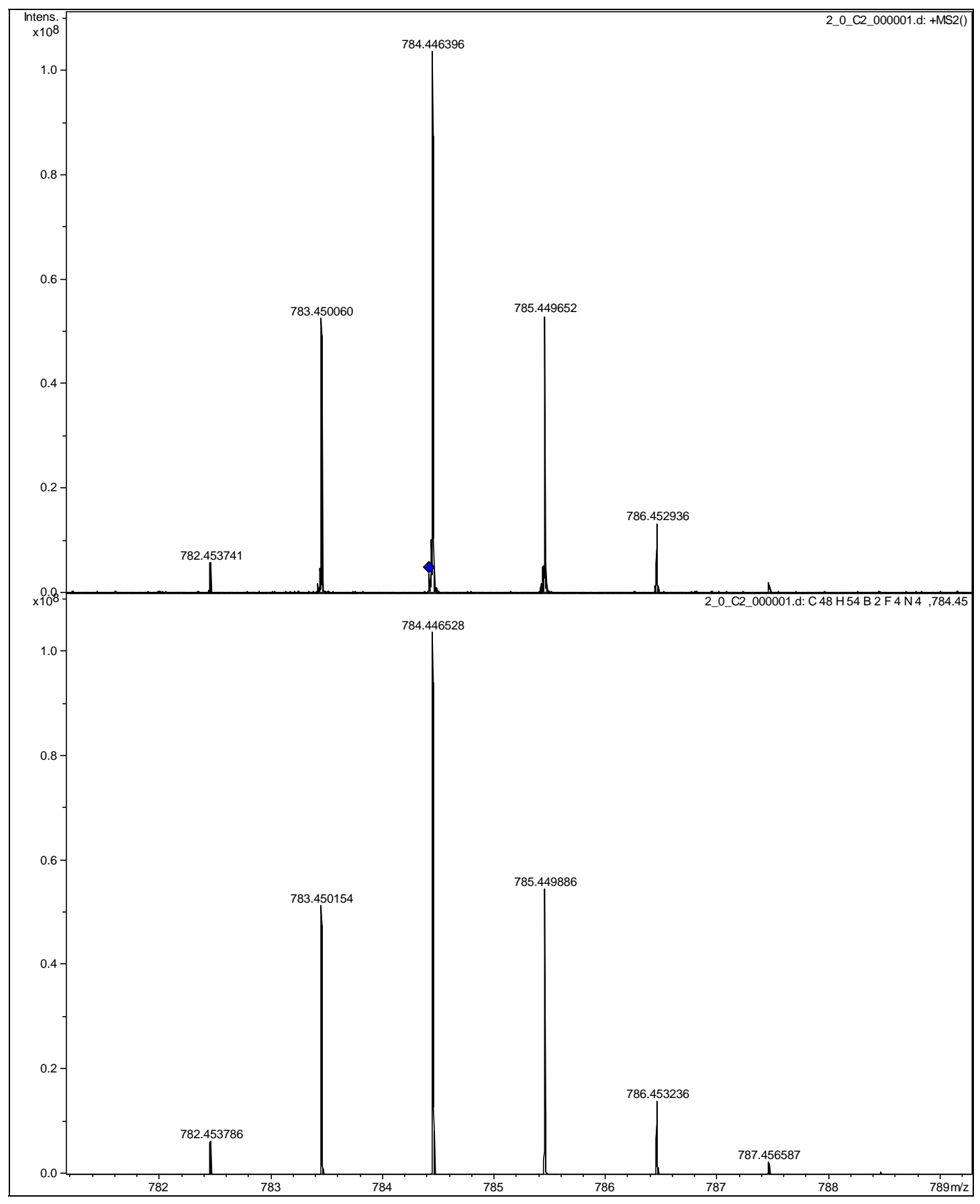

Observed (top) and calculated (bottom) HRMS for 2. 


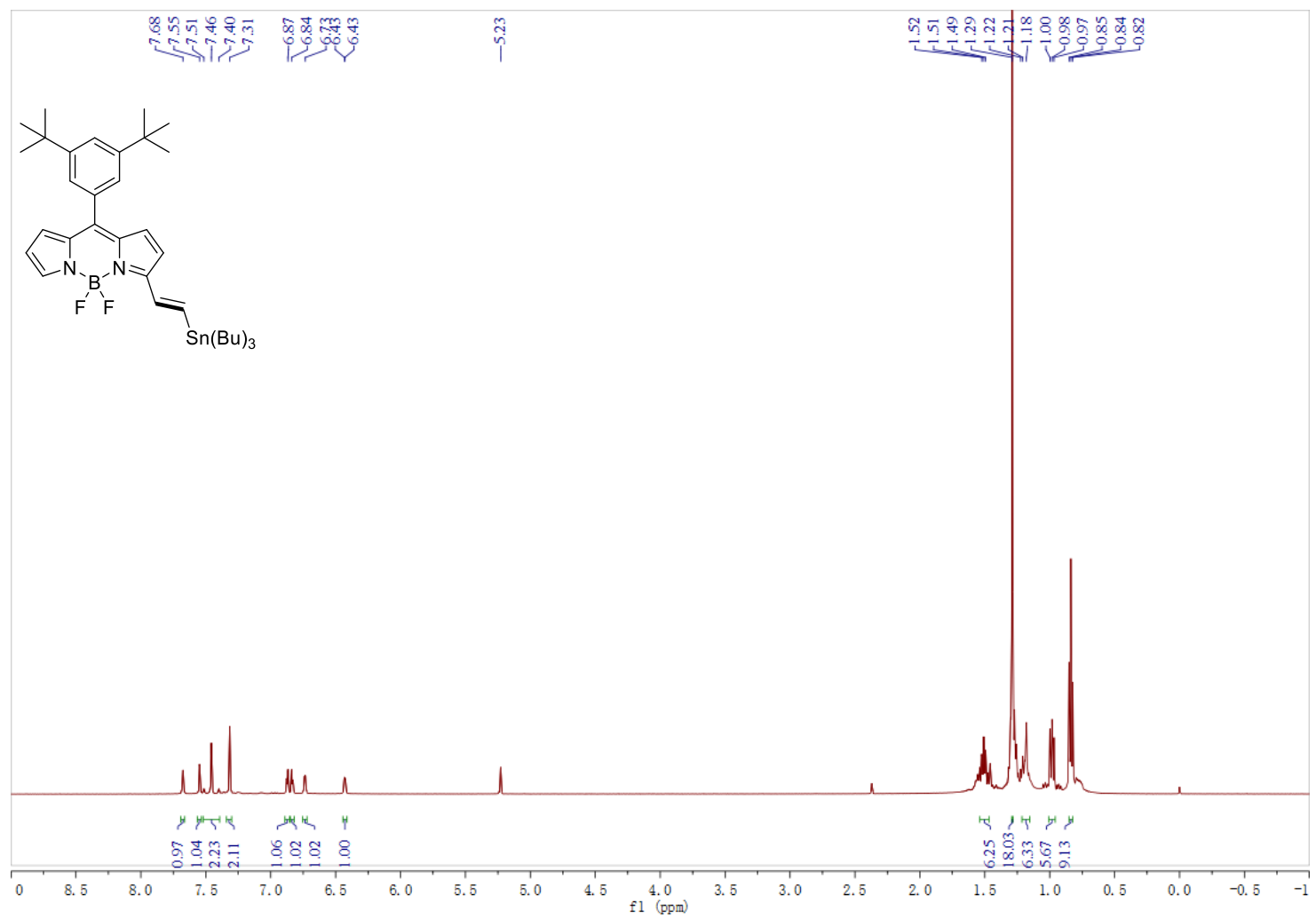

${ }^{1} \mathrm{H}$ NMR (500 MHz) spectrum of $\mathbf{1 S n}$ in $\mathrm{CD}_{2} \mathrm{Cl}_{2}$.
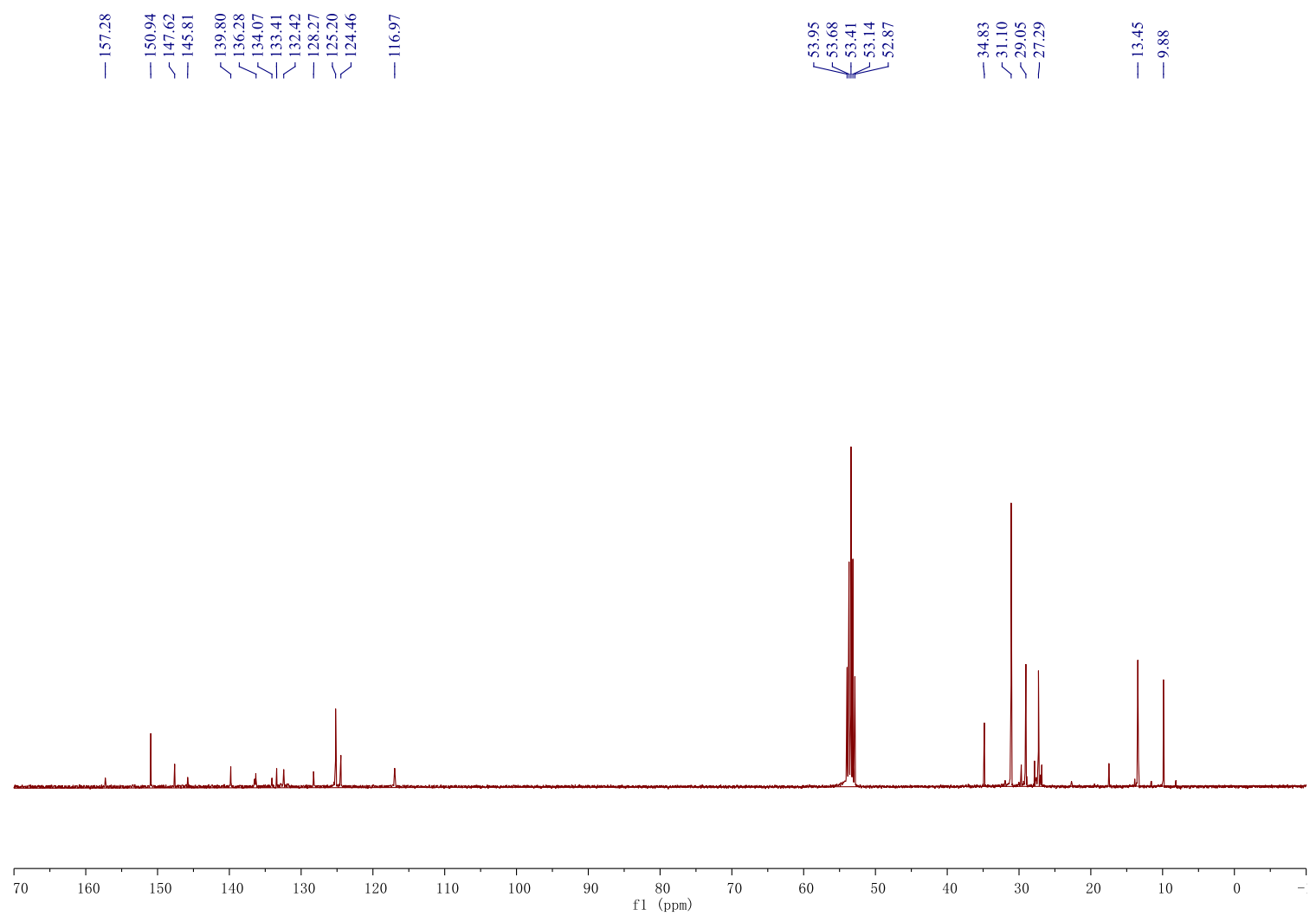

${ }^{13} \mathrm{C}$ NMR (400 MHz) spectrum of $\mathbf{1 S n}$ in $\mathrm{CD}_{2} \mathrm{Cl}_{2}$. 


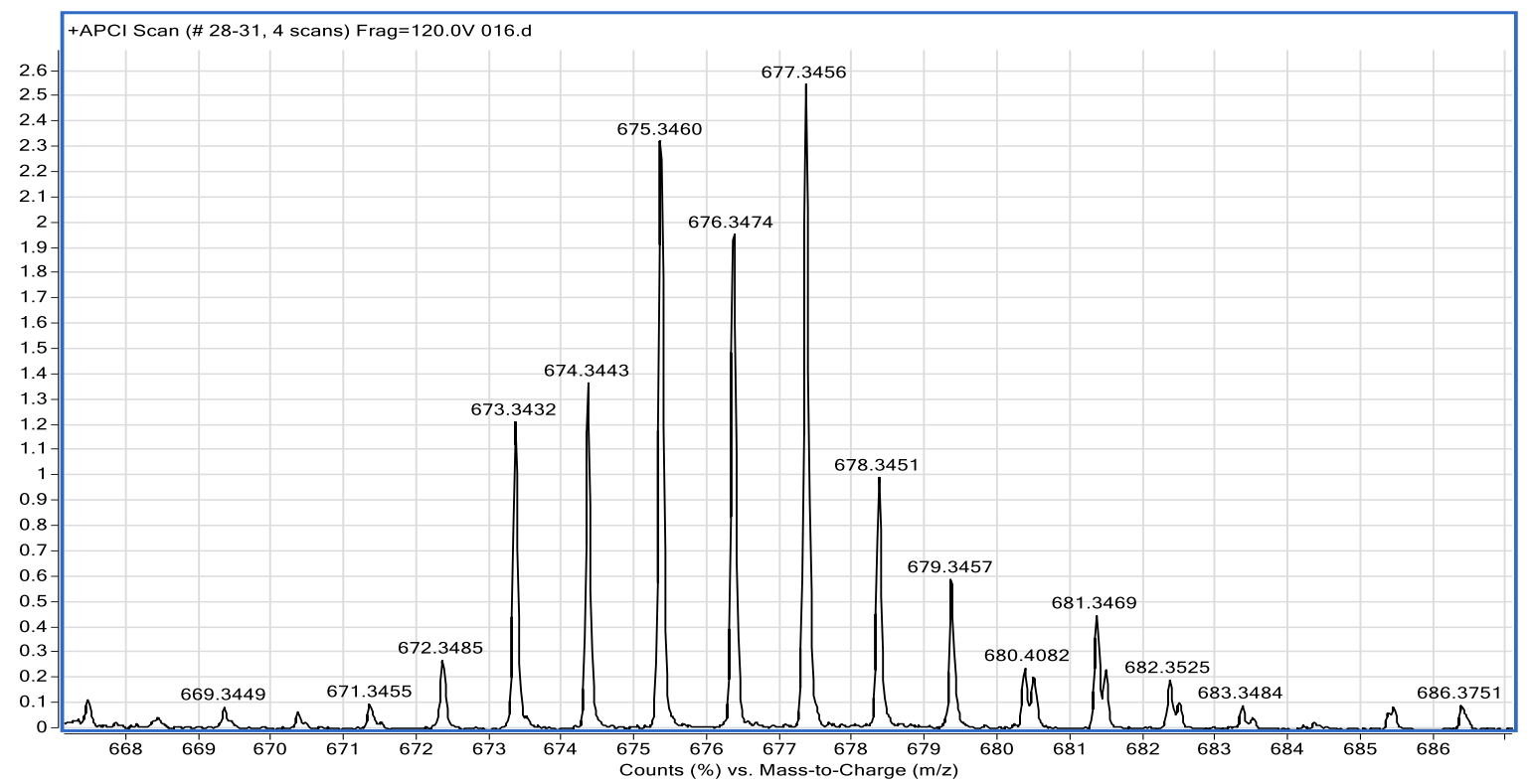

Observed HRMS for 1Sn. 


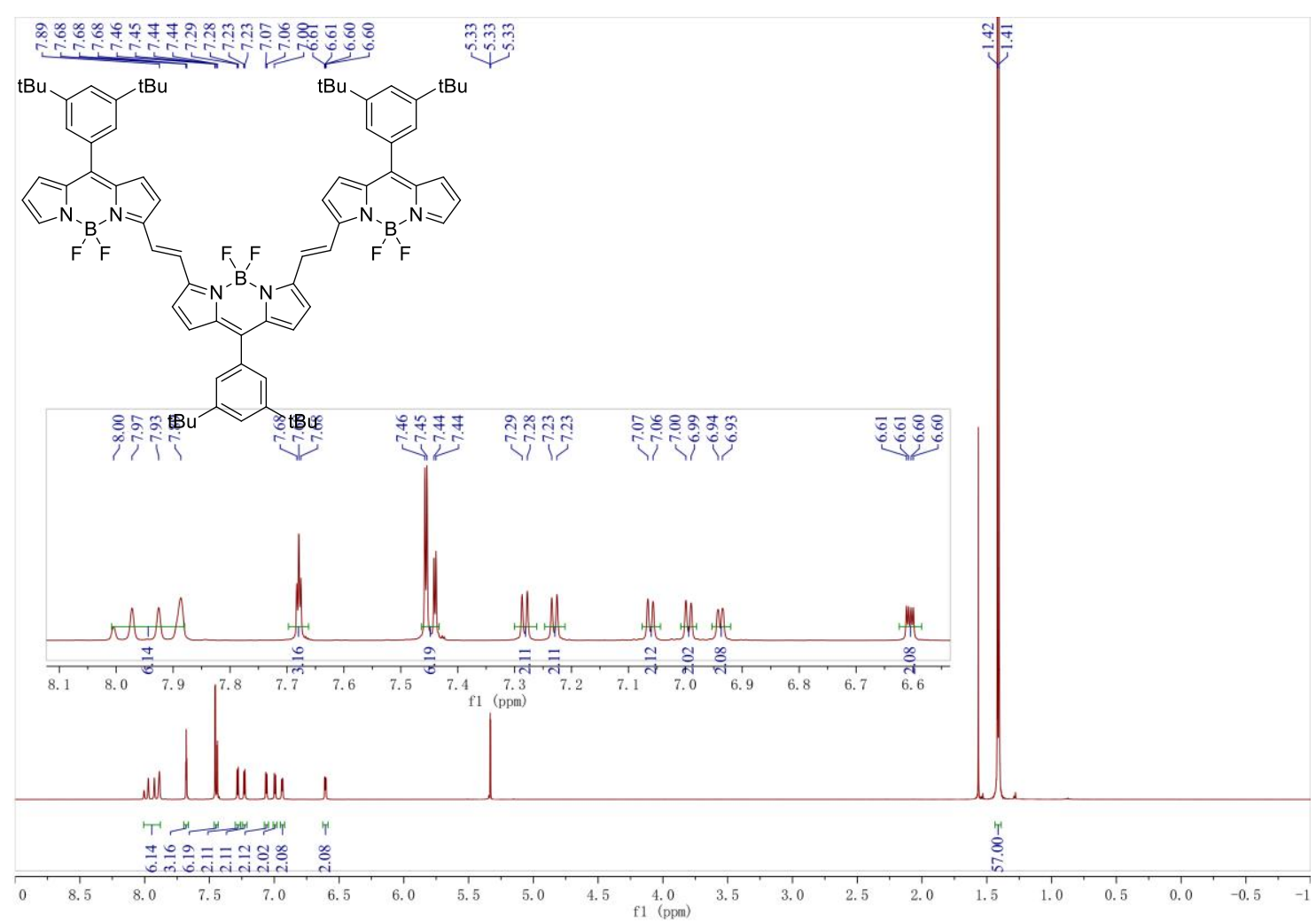

${ }^{1} \mathrm{H}$ NMR (500 MHz) spectrum of 3 in $\mathrm{CD}_{2} \mathrm{Cl}_{2}$.
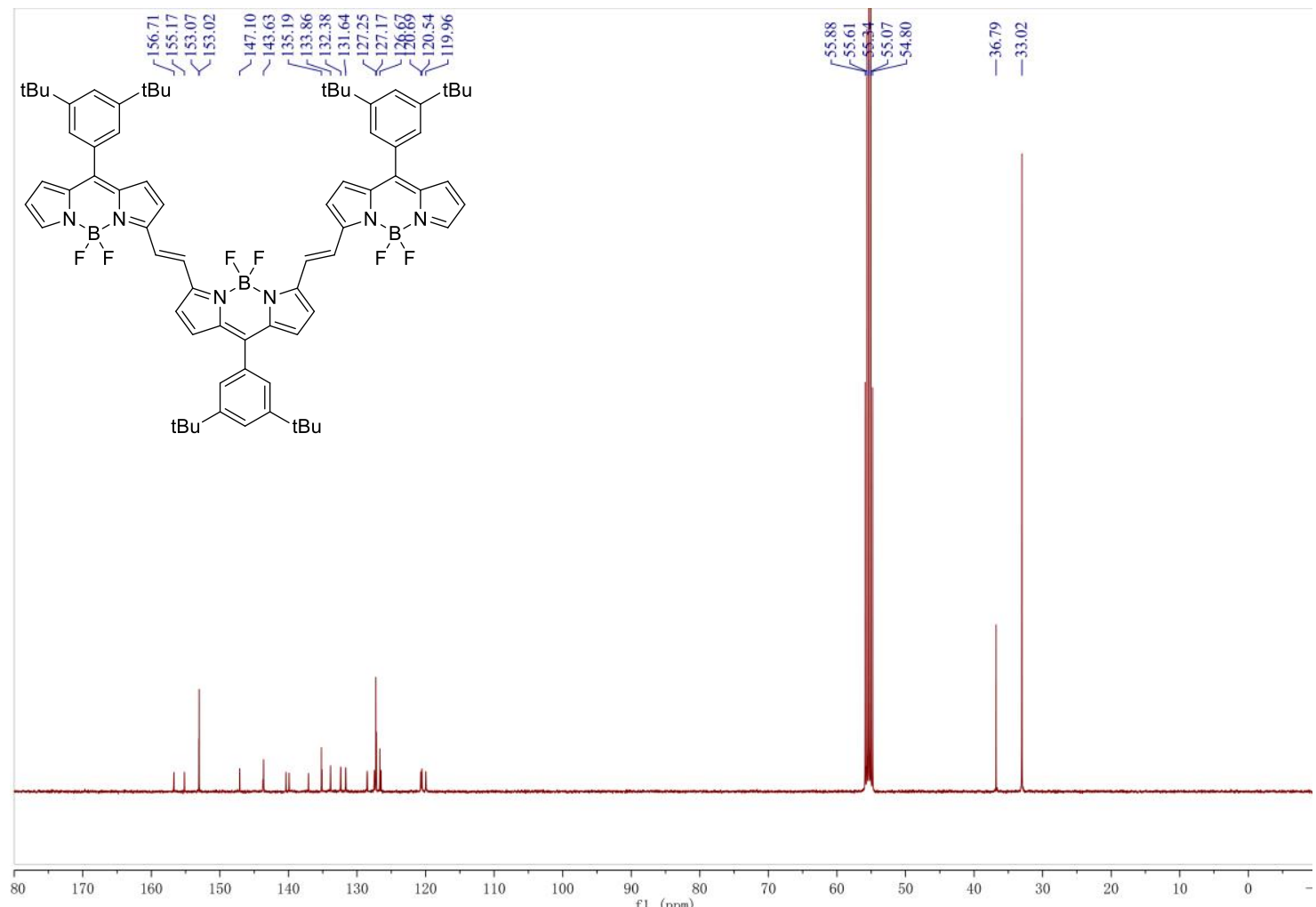

${ }^{13} \mathrm{C}$ NMR (400 MHz) spectrum of 3 in $\mathrm{CD}_{2} \mathrm{Cl}_{2}$. 


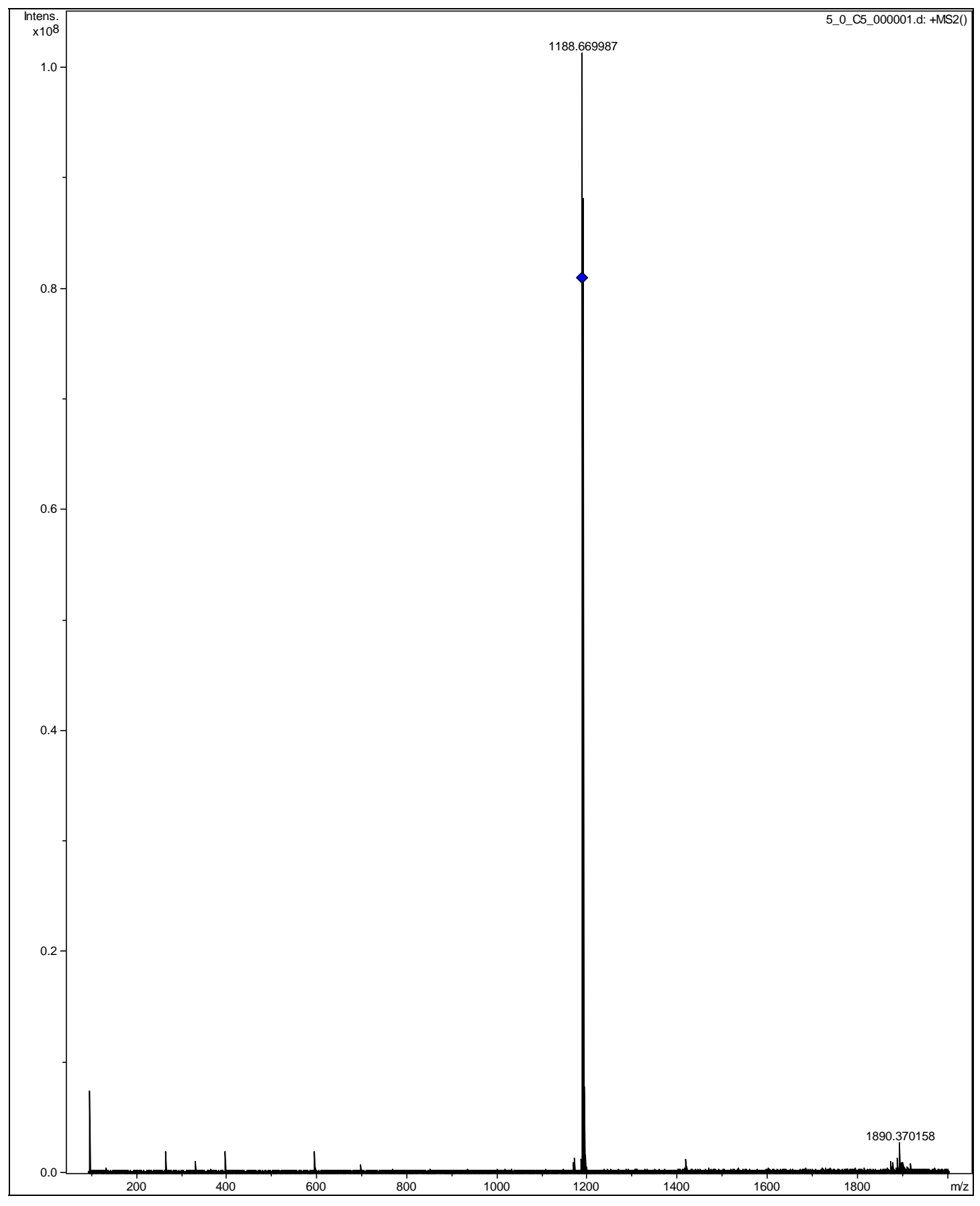

HRMS for 3. 


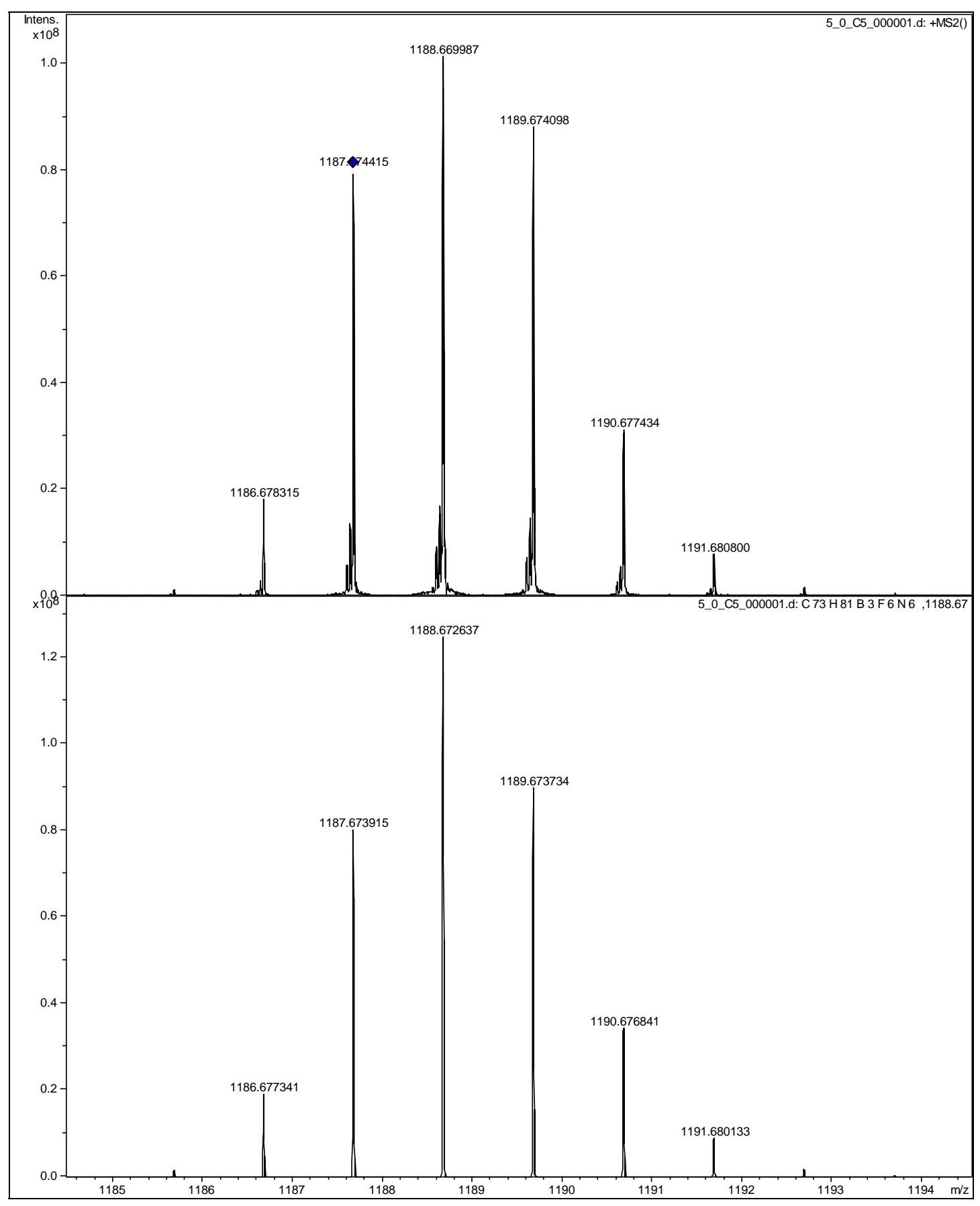

Observed (top) and calculated (bottom) HRMS for 3. 


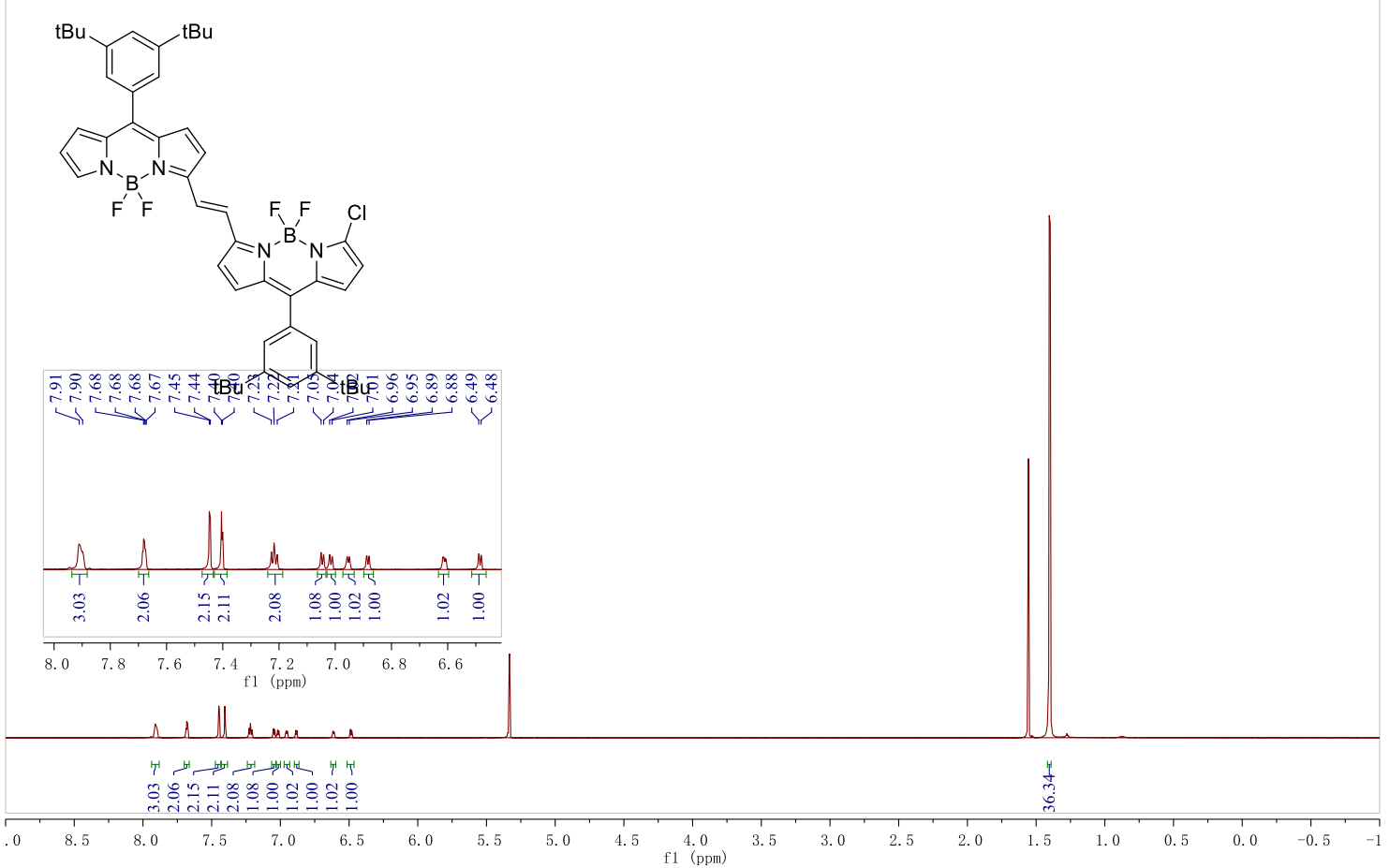

${ }^{1} \mathrm{H}$ NMR (500 MHz) spectrum of $\mathbf{2 C l}$ in $\mathrm{CD}_{2} \mathrm{Cl}_{2}$.

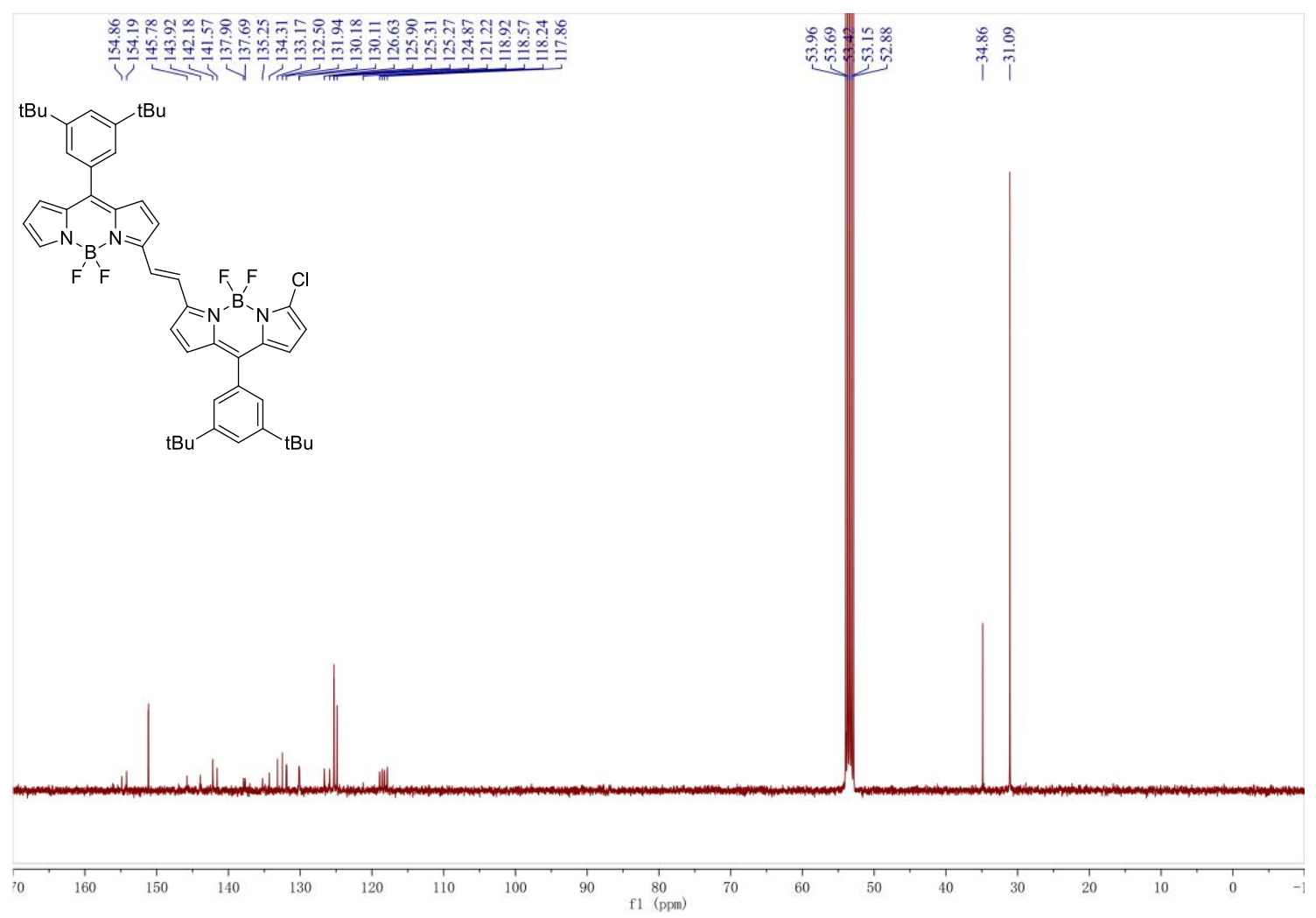

${ }^{13} \mathrm{C}$ NMR (400 MHz) spectrum of $\mathbf{2 C l}$ in $\mathrm{CD}_{2} \mathrm{Cl}_{2}$. 


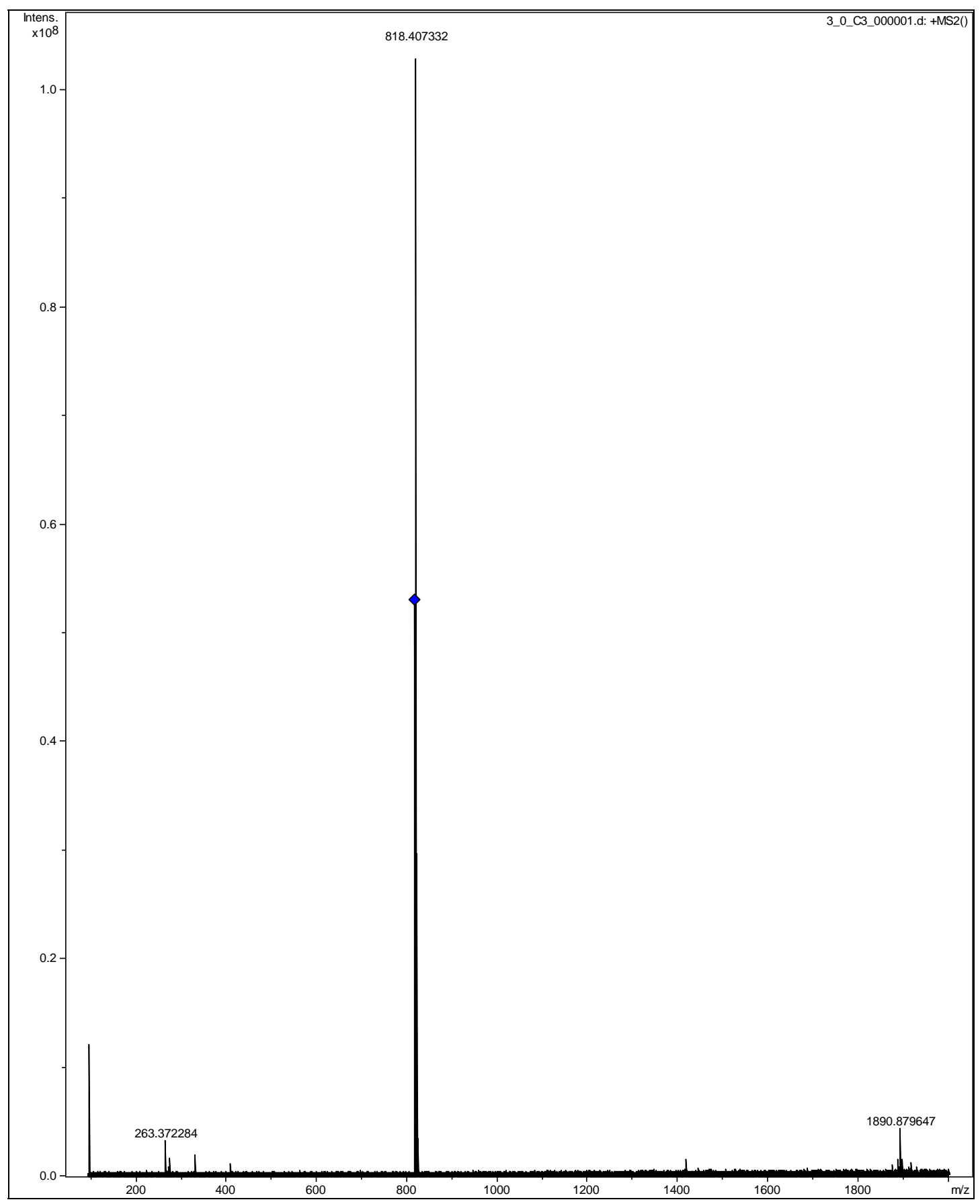

HRMS for $\mathbf{2 C l}$. 


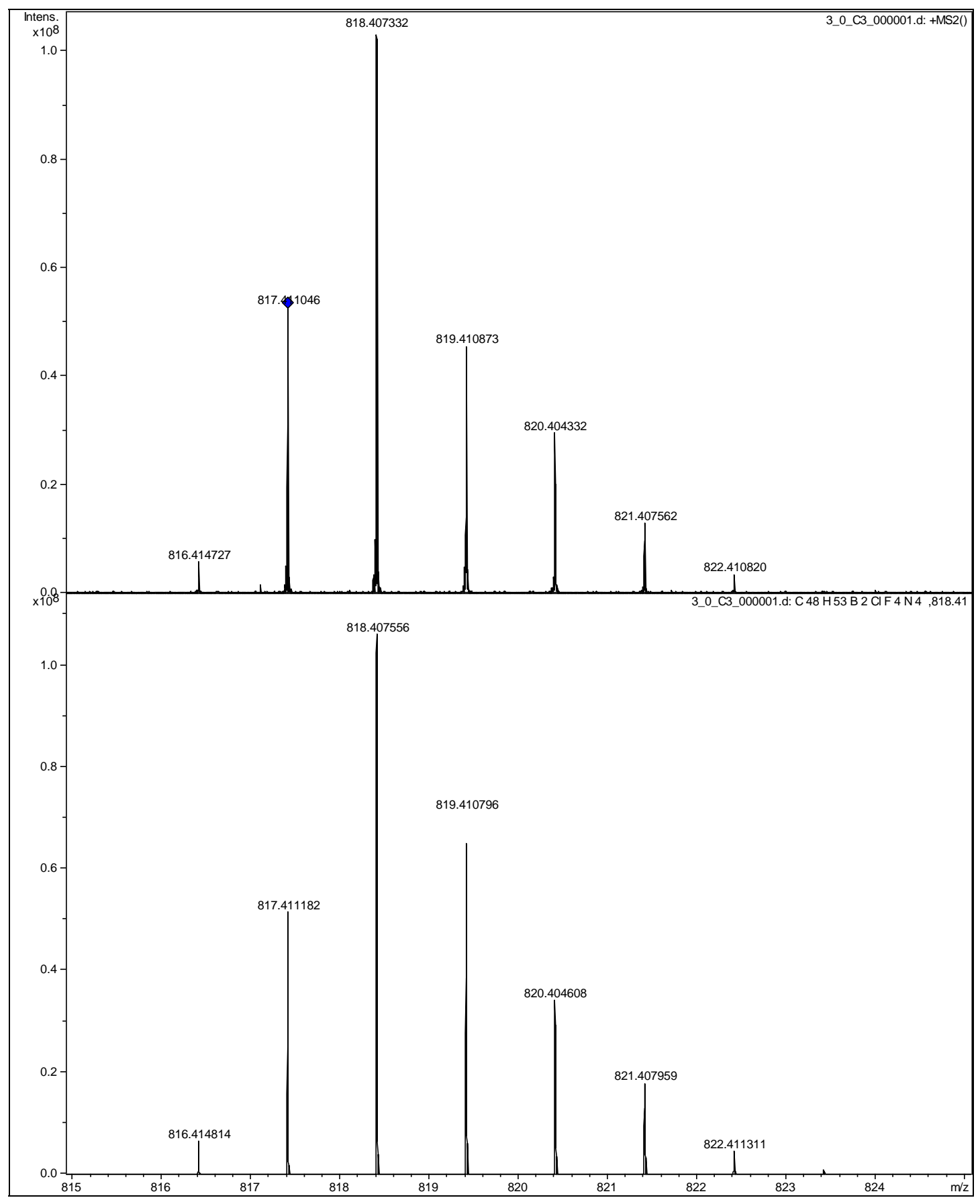

Observed (top) and calculated (bottom) HRMS for $\mathbf{2 C l}$. 


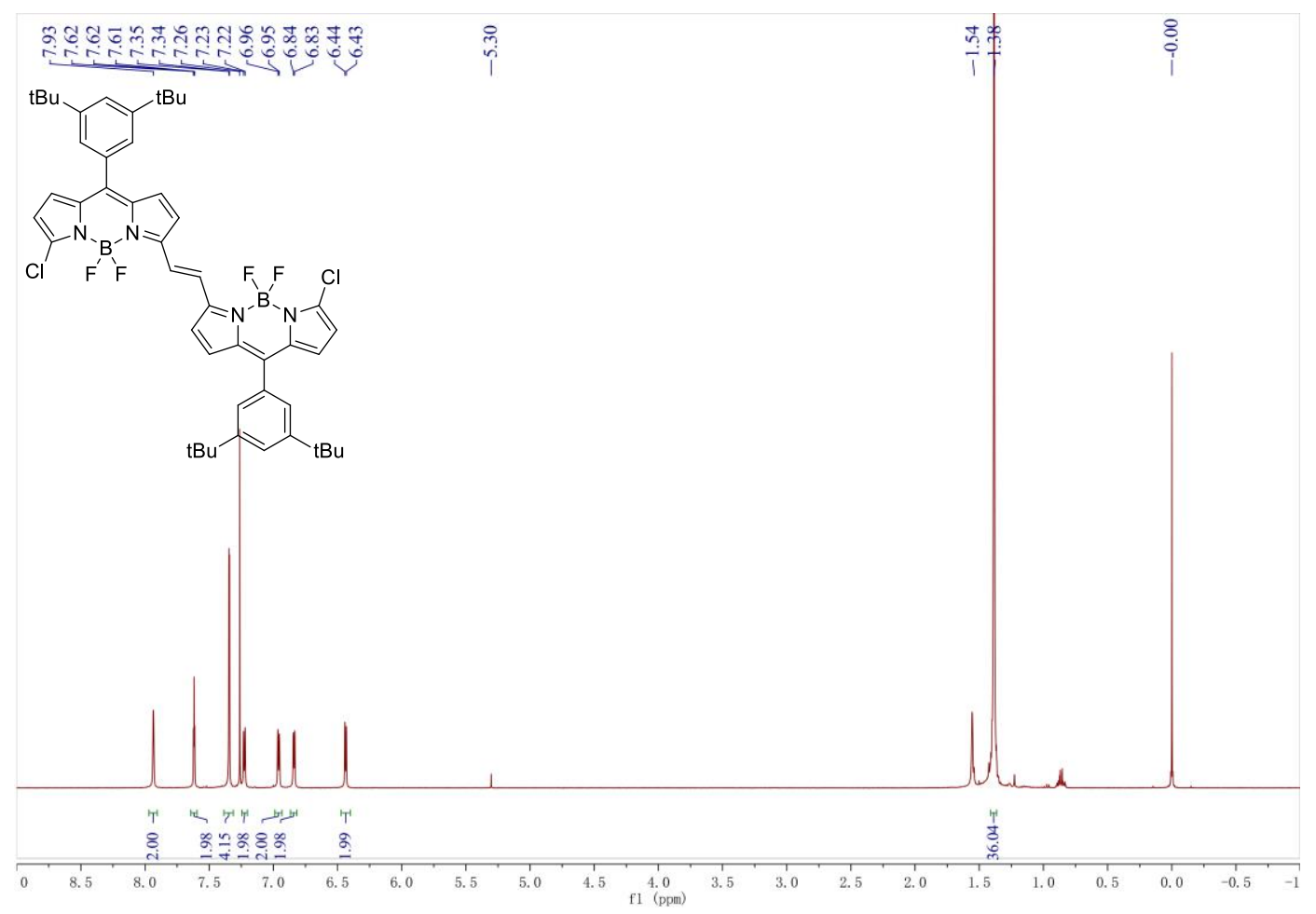

${ }^{1} \mathrm{H}$ NMR (400 MHz) spectrum of $\mathbf{2} \mathbf{C l}_{2}$ in $\mathrm{CD}_{2} \mathrm{Cl}_{2}$.
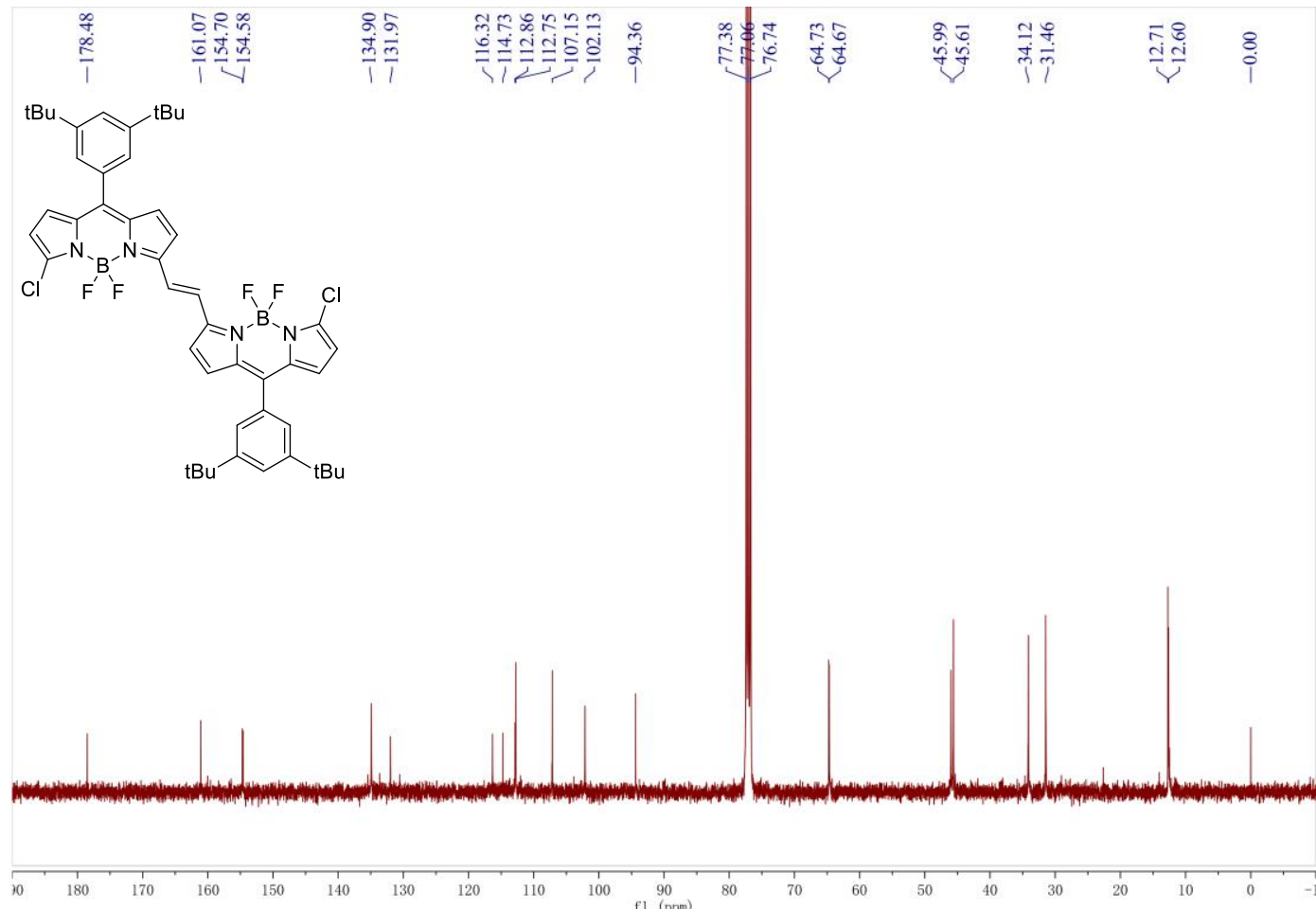

${ }^{13} \mathrm{C}$ NMR (400 MHz) spectrum of $\mathbf{2} \mathbf{C l}_{\mathbf{2}}$ in $\mathrm{CD}_{2} \mathrm{Cl}_{2}$. 


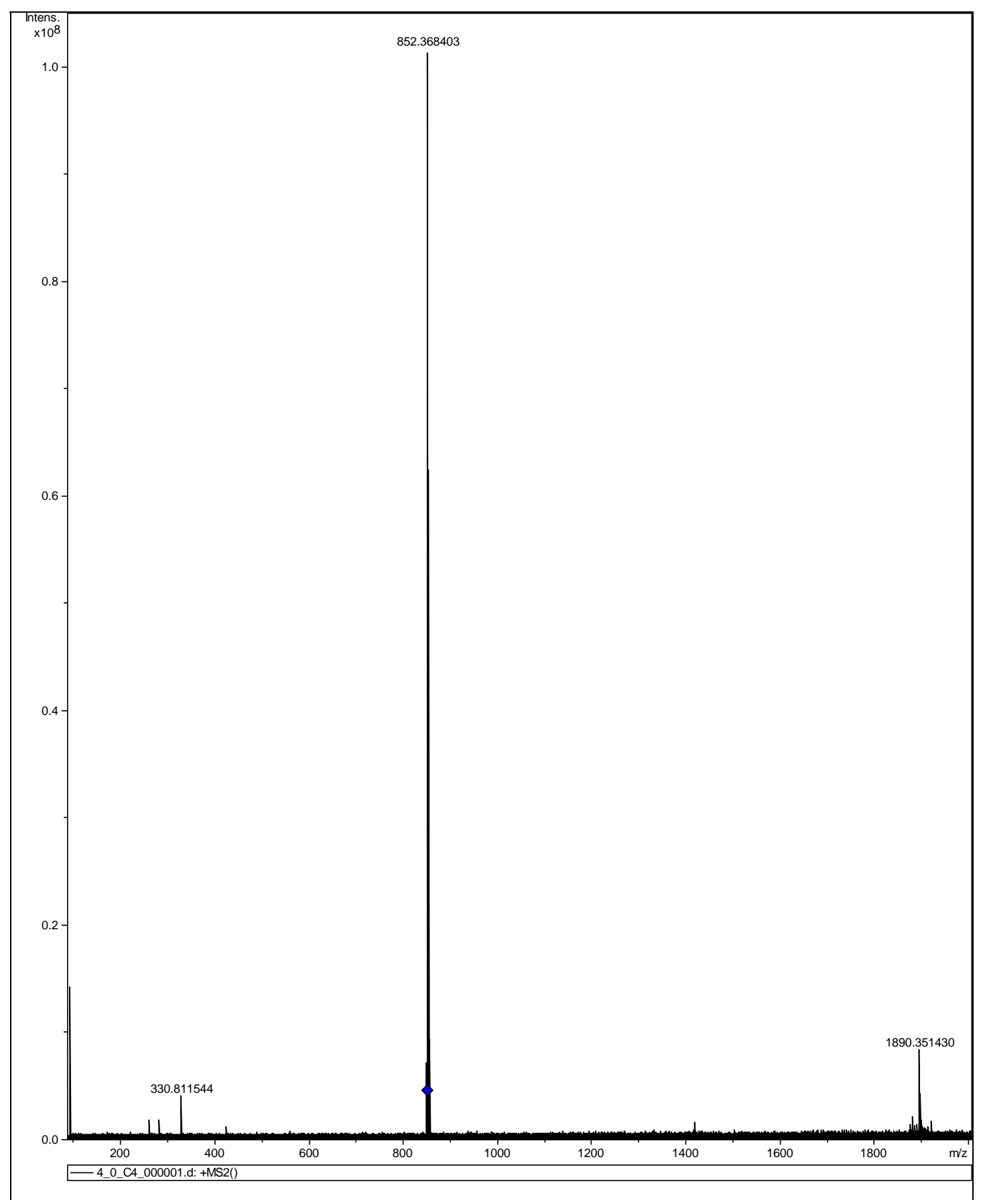

HRMS for $\mathbf{2 C l}$. 


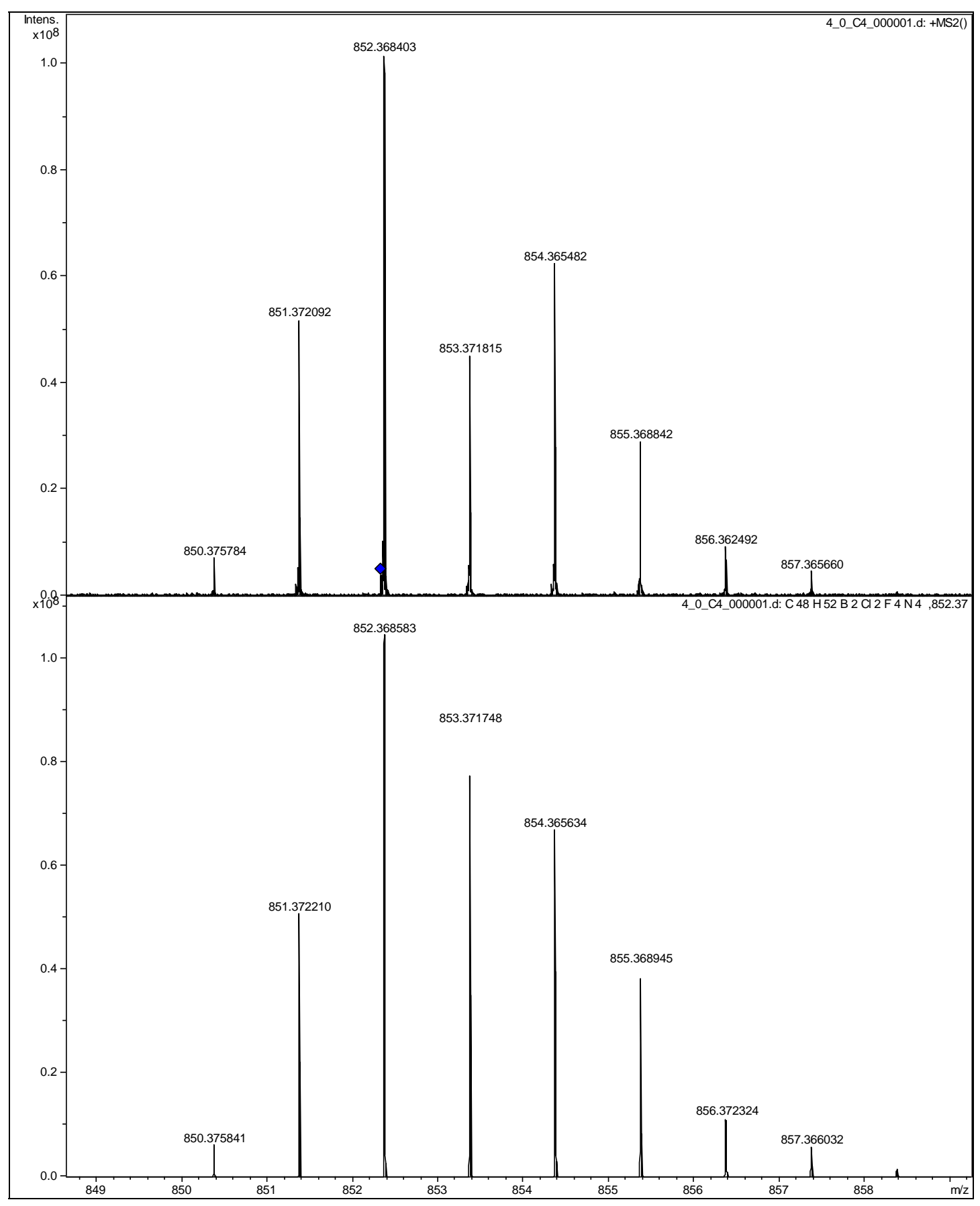

Observed (top) and calculated (bottom) HRMS for $\mathbf{2 C l}$. 


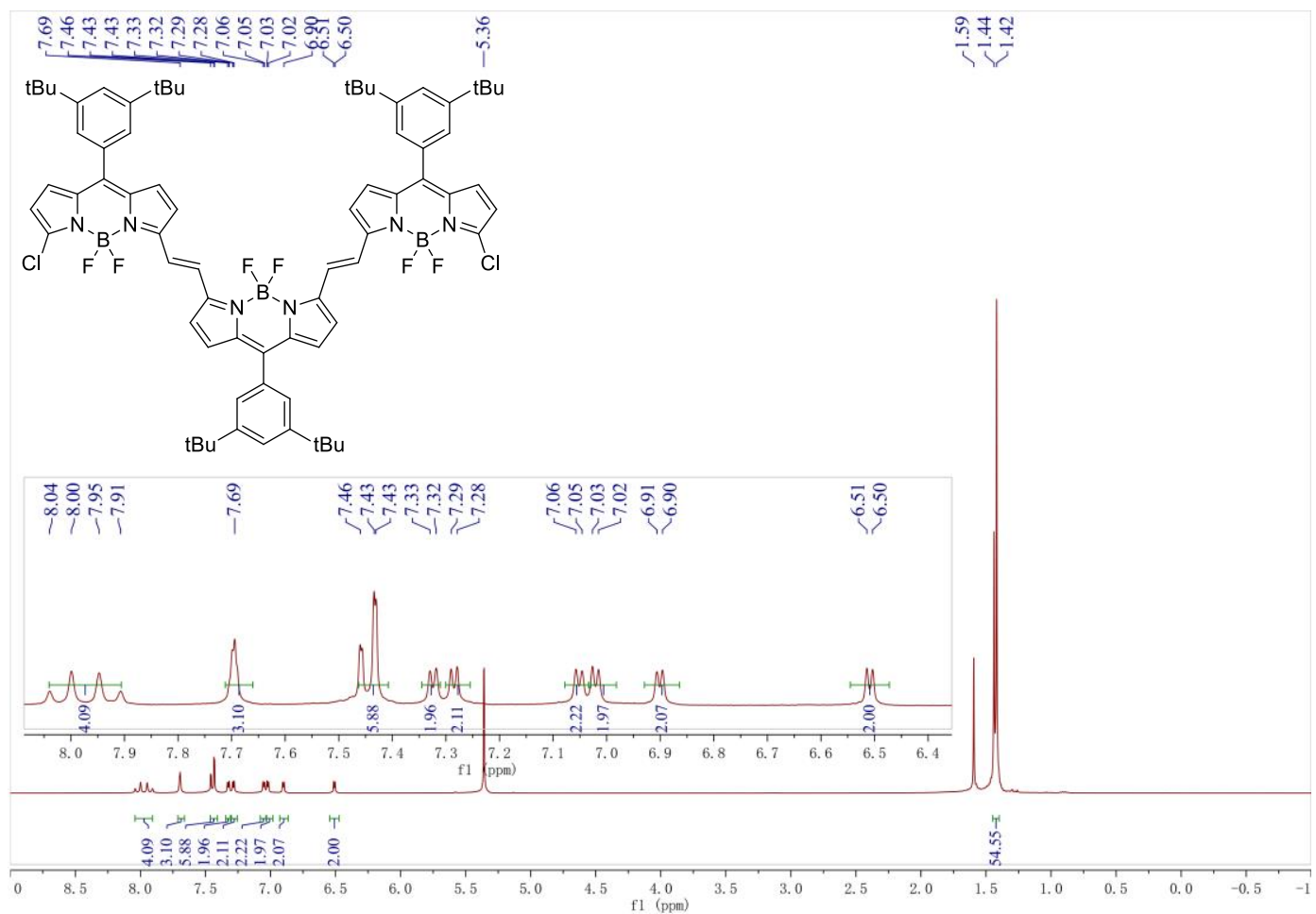

${ }^{1} \mathrm{H}$ NMR (400 MHz) spectrum of $\mathbf{3} \mathbf{C l}_{2}$ in $\mathrm{CD}_{2} \mathrm{Cl}_{2}$.
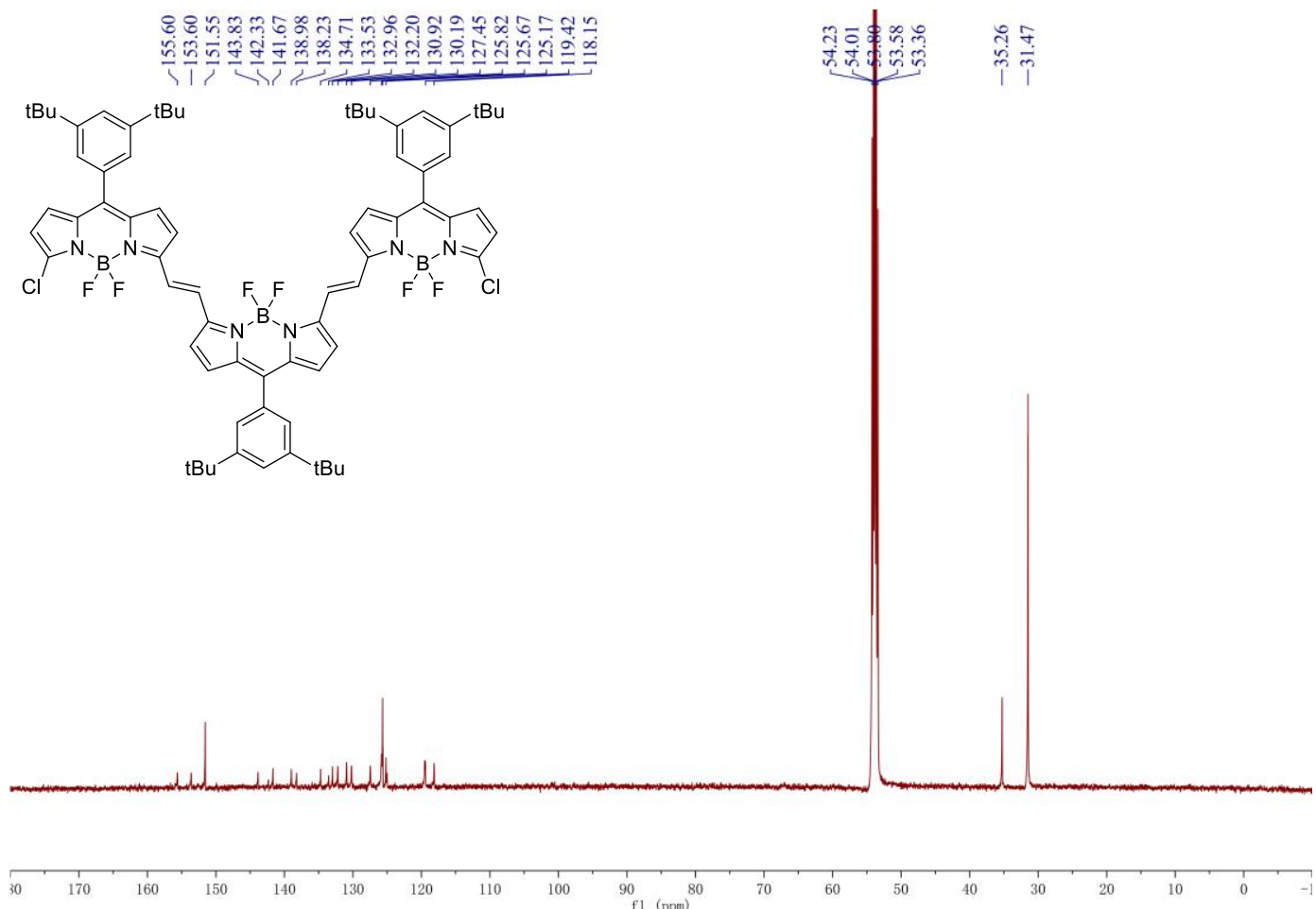

${ }^{13} \mathrm{C}$ NMR (500 MHz) spectrum of $\mathbf{3} \mathbf{C l}_{2}$ in $\mathrm{CD}_{2} \mathrm{Cl}_{2}$. 


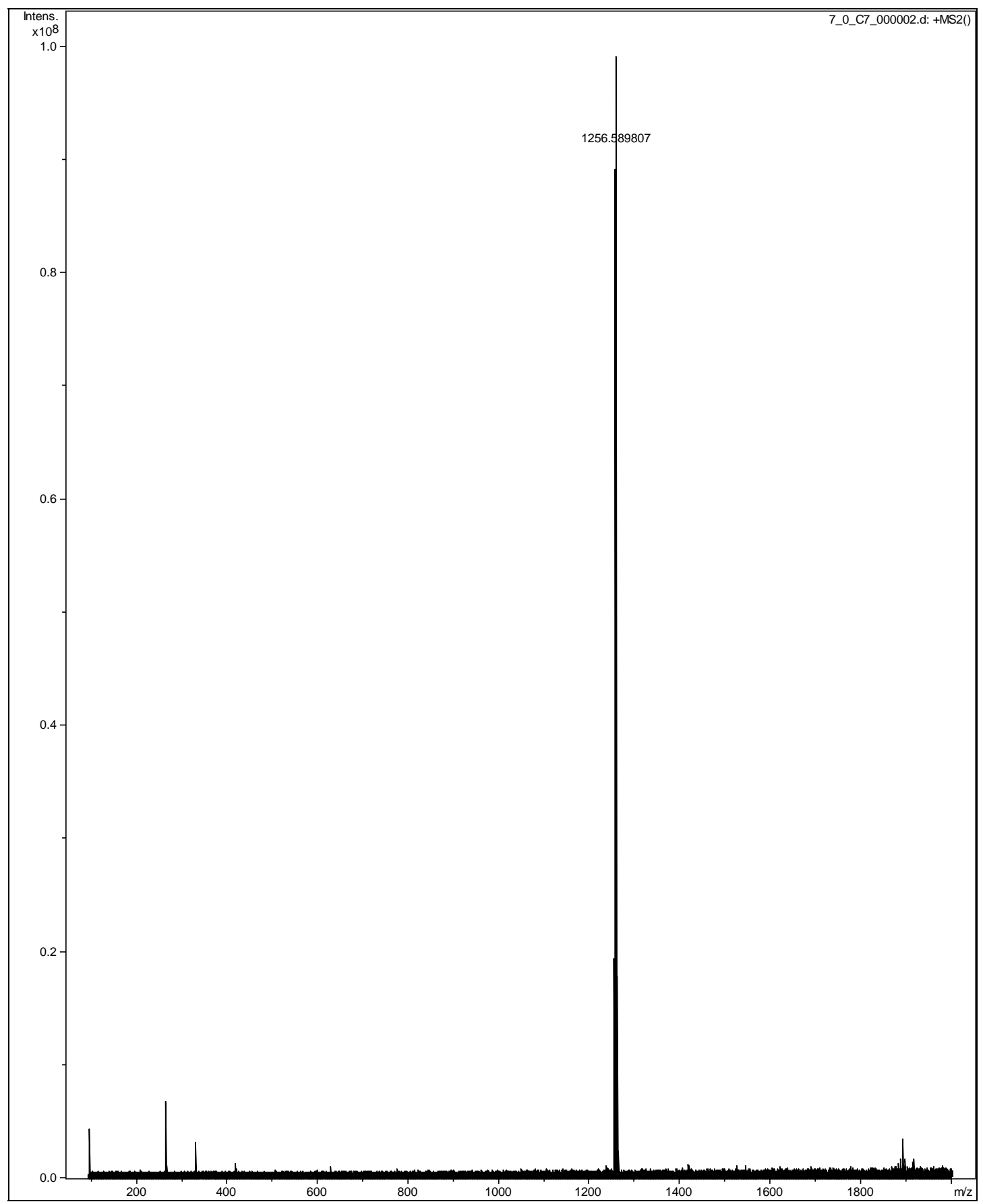

HRMS for 3Cl2. 


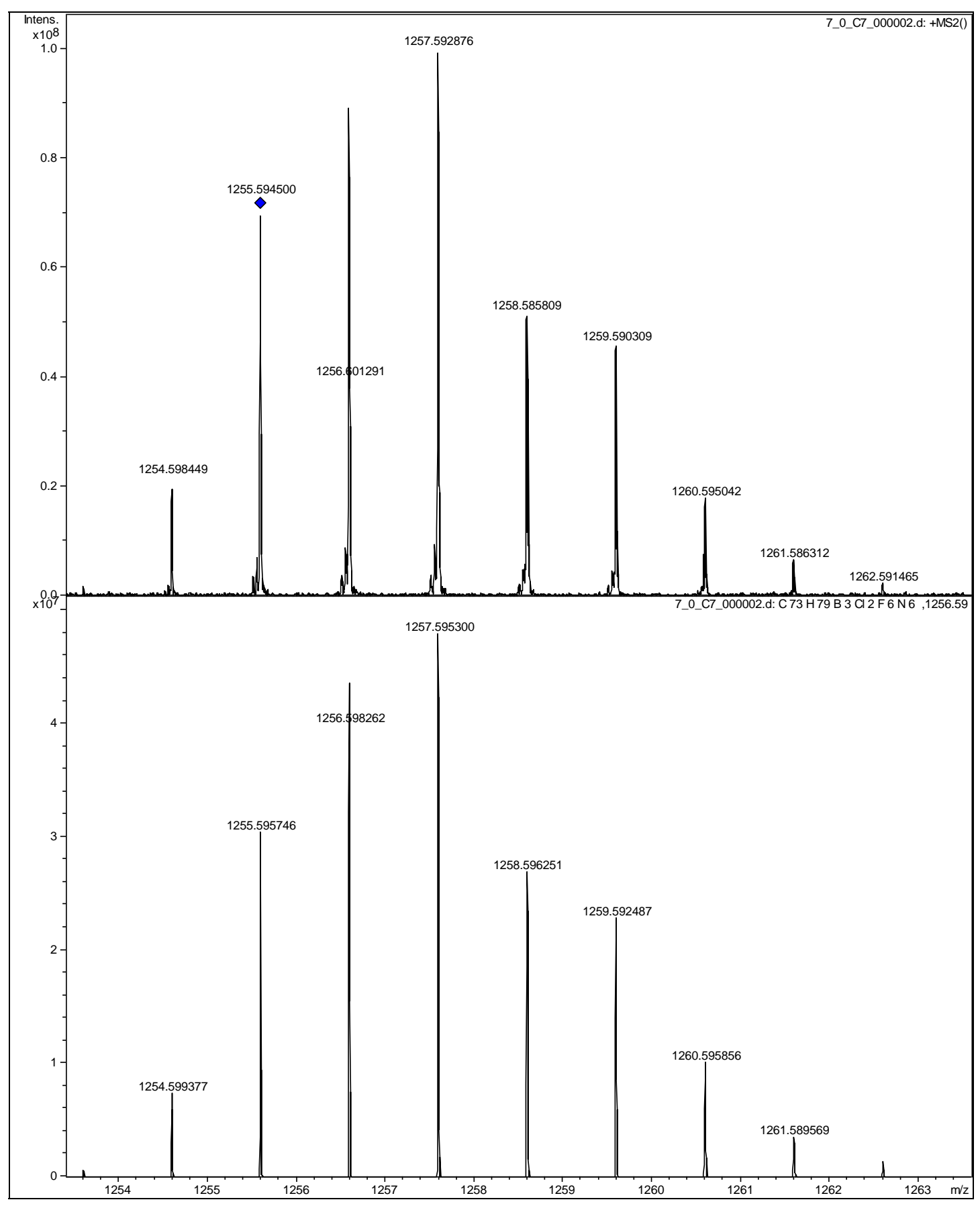

Observed (top) and calculated (bottom) HRMS for $\mathbf{3 C l}$. 


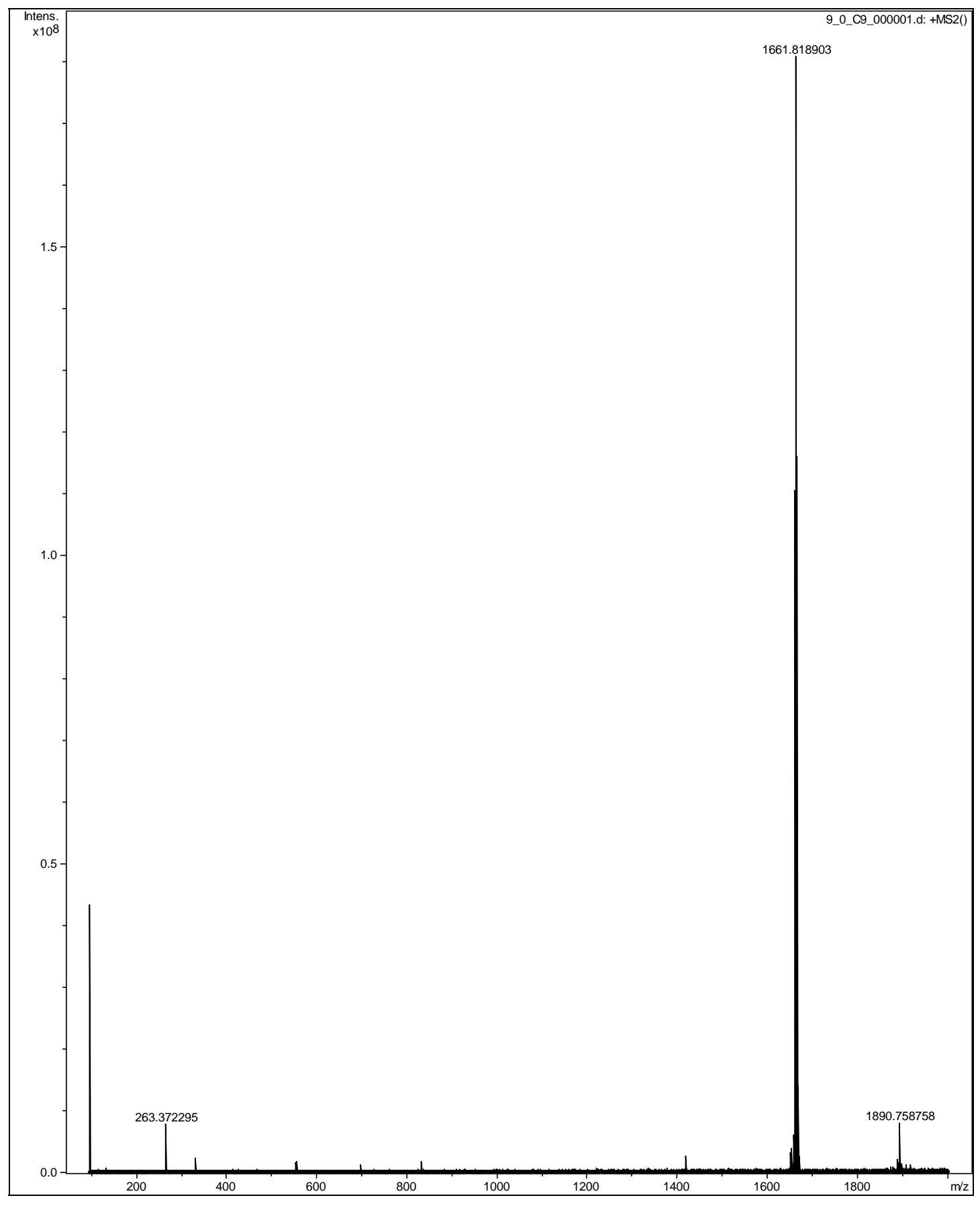

HRMS for $\mathbf{4 C l}$. 


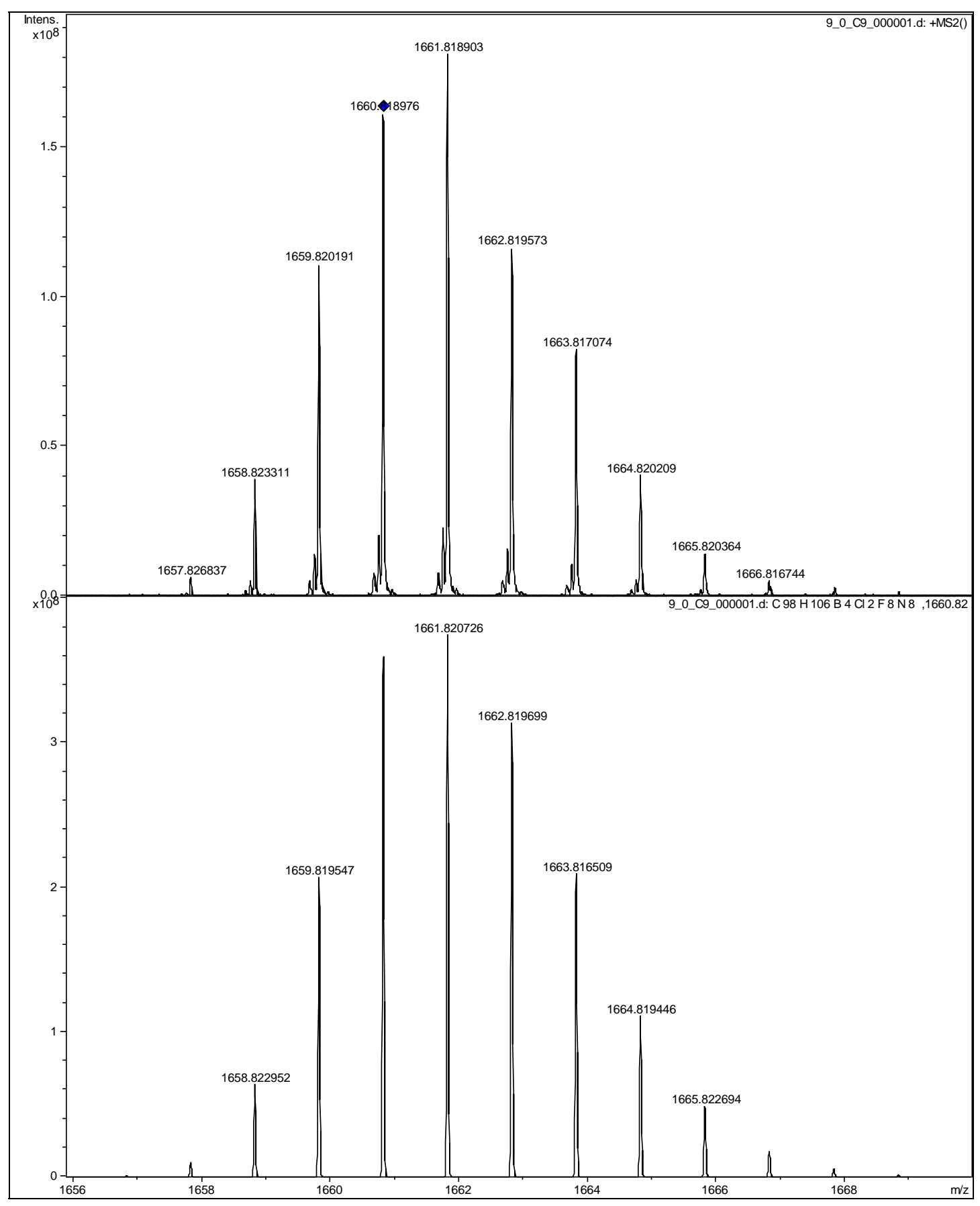

Observed (top) and calculated (bottom) HRMS for $\mathbf{4 C l}$. 


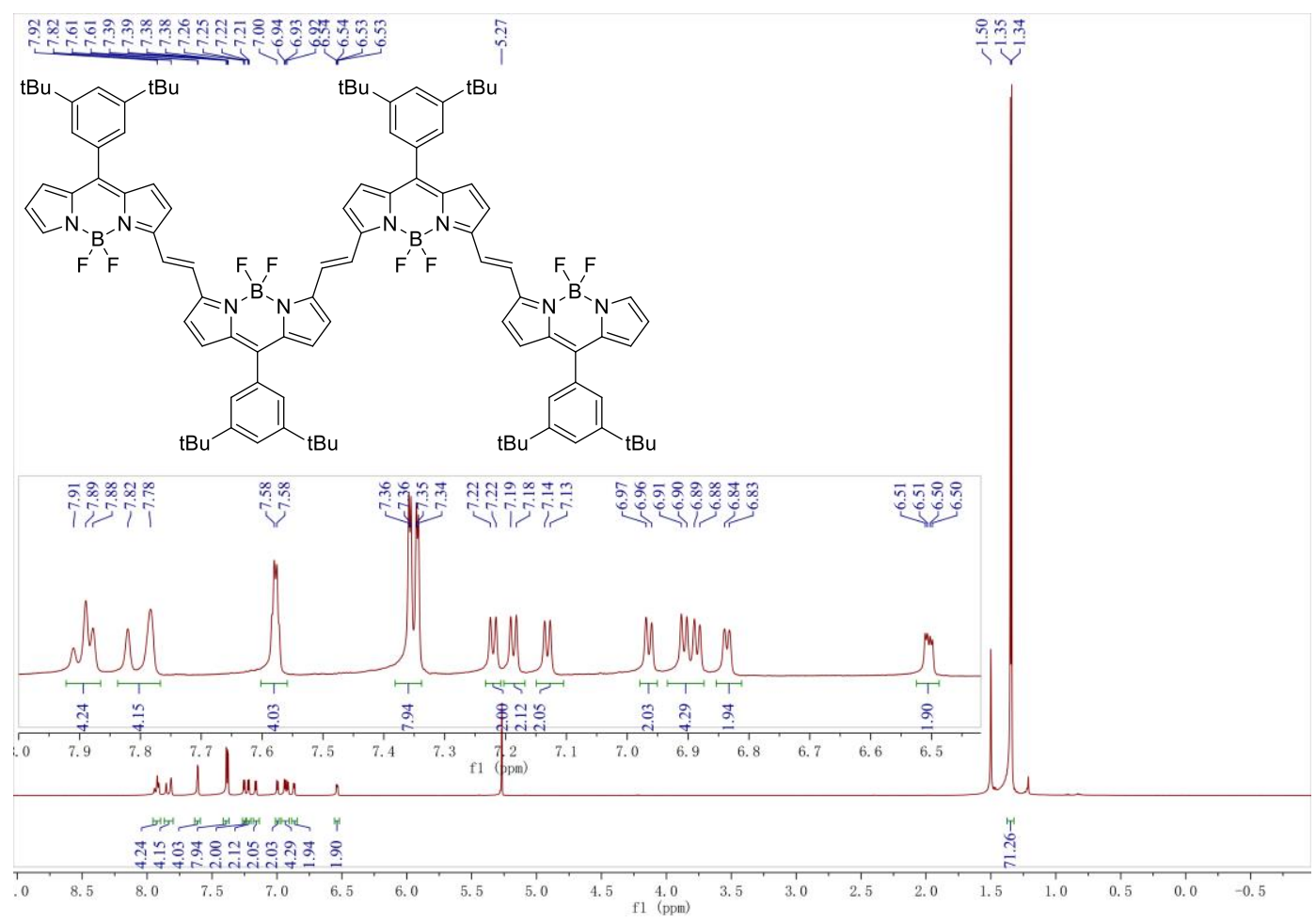

${ }^{1} \mathrm{H}$ NMR (500 MHz) spectrum of 4 in $\mathrm{CD}_{2} \mathrm{Cl}_{2}$.

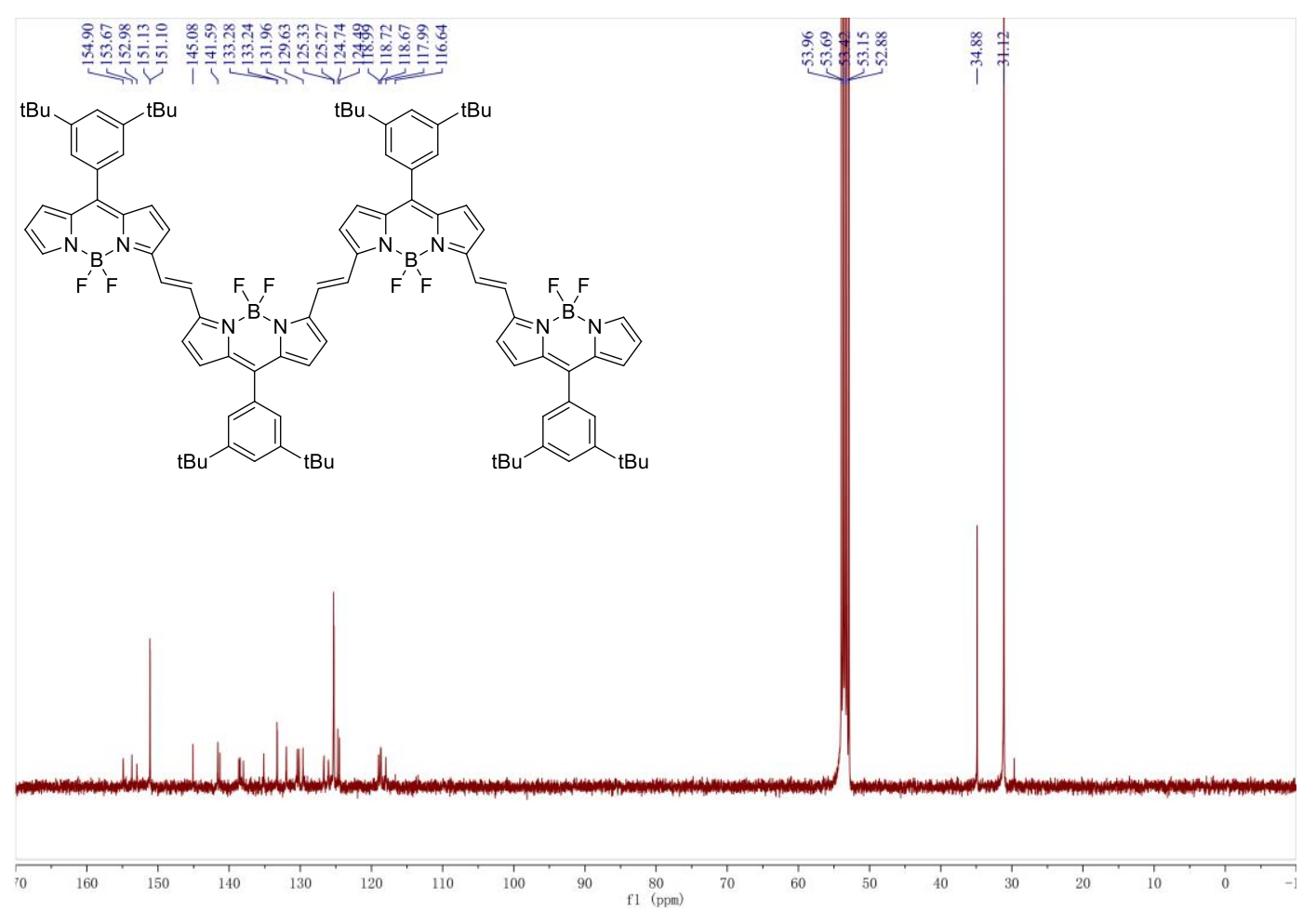

${ }^{13} \mathrm{C}$ NMR (400 MHz) spectrum of 4 in $\mathrm{CD}_{2} \mathrm{Cl}_{2}$. 


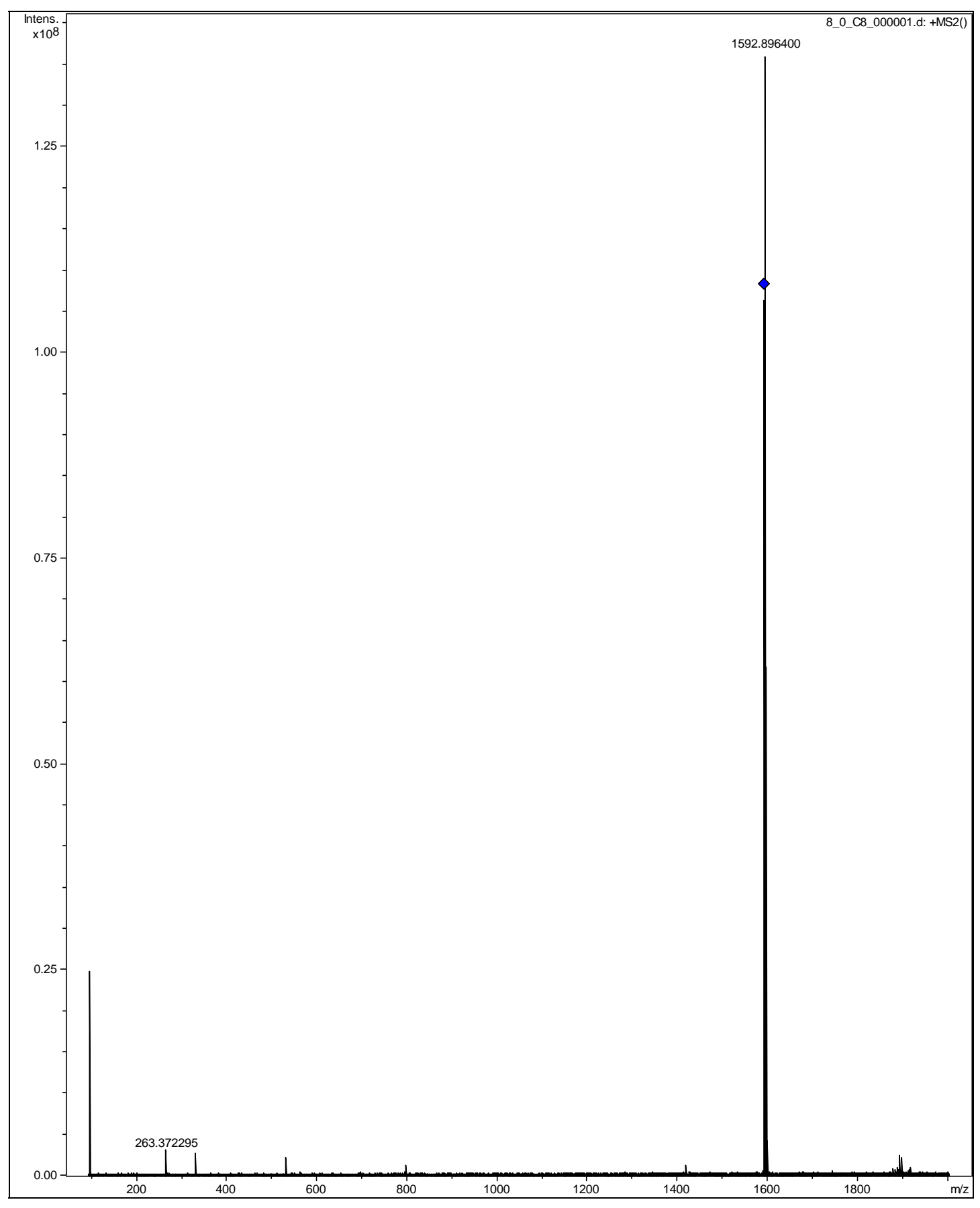

HRMS for 4. 


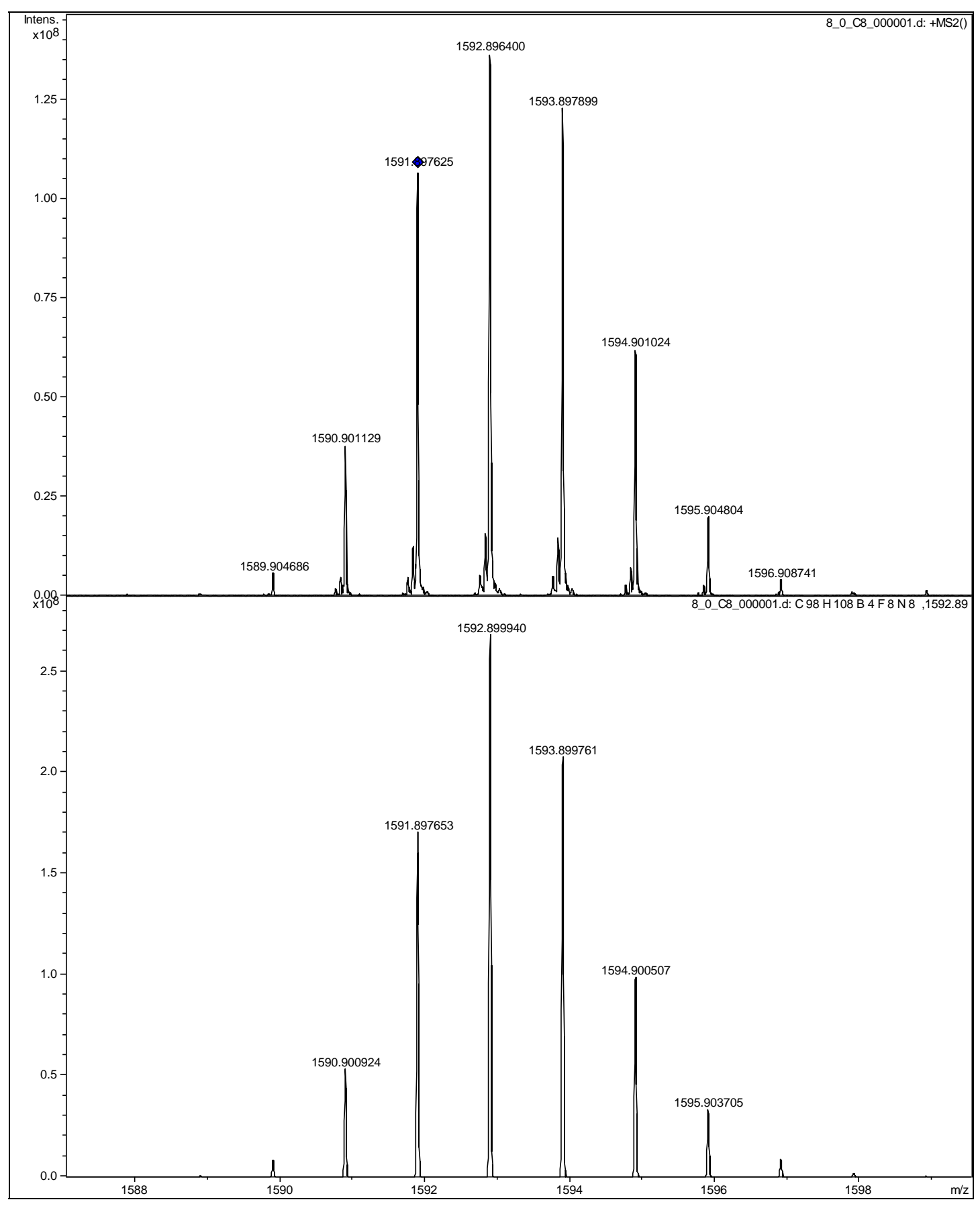

Observed (top) and calculated (bottom) HRMS for 4. 


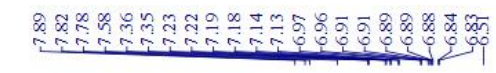

$$
\text { i }
$$

देले

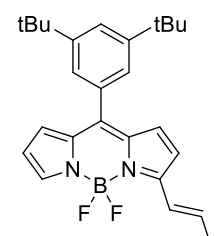

${ }^{\mathrm{tBu}} \mathrm{C}^{\mathrm{tBu}}$

$F$
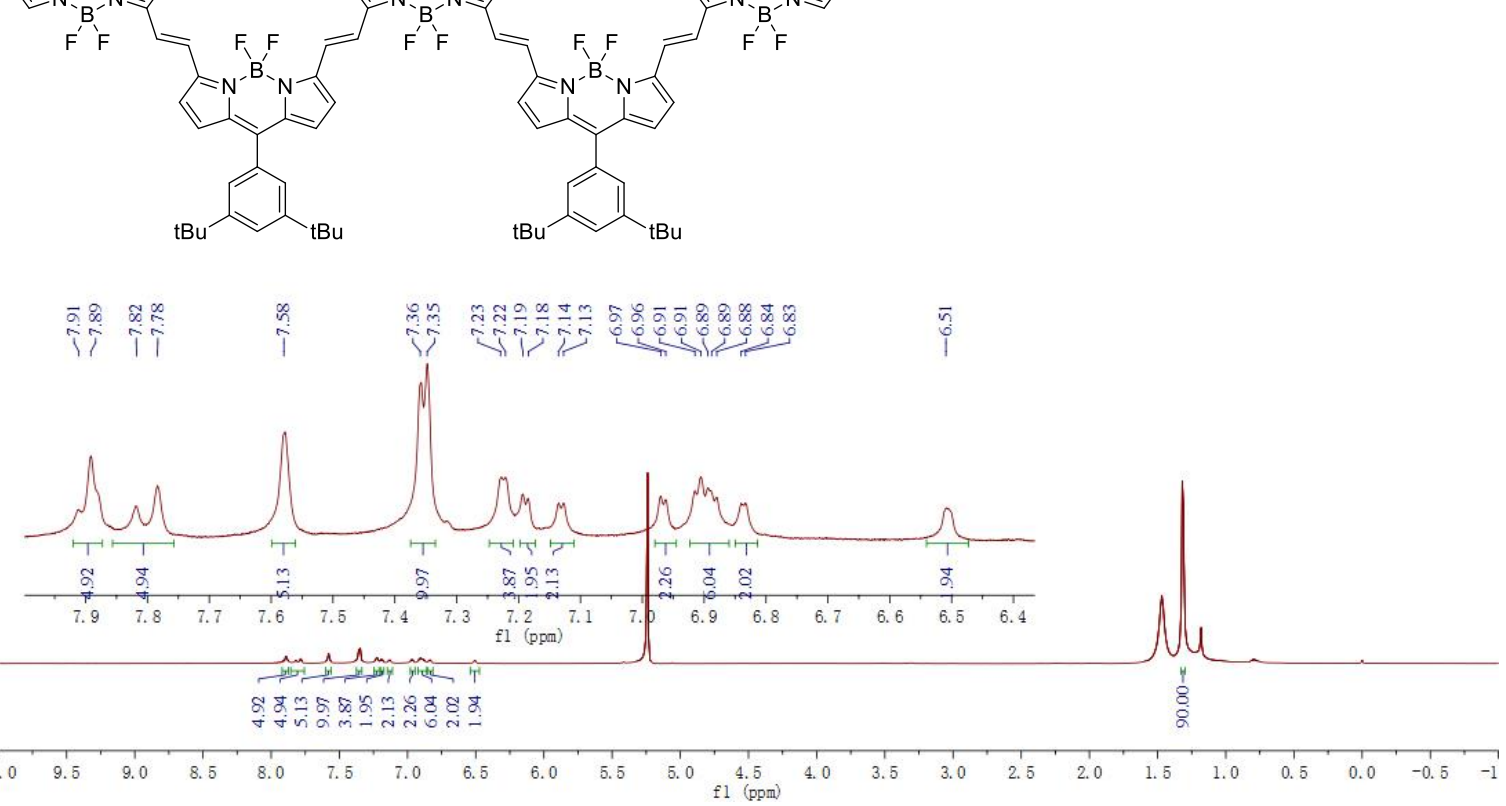

${ }^{1} \mathrm{H}$ NMR (500 MHz) spectrum of 5 in $\mathrm{CD}_{2} \mathrm{Cl}_{2}$. 


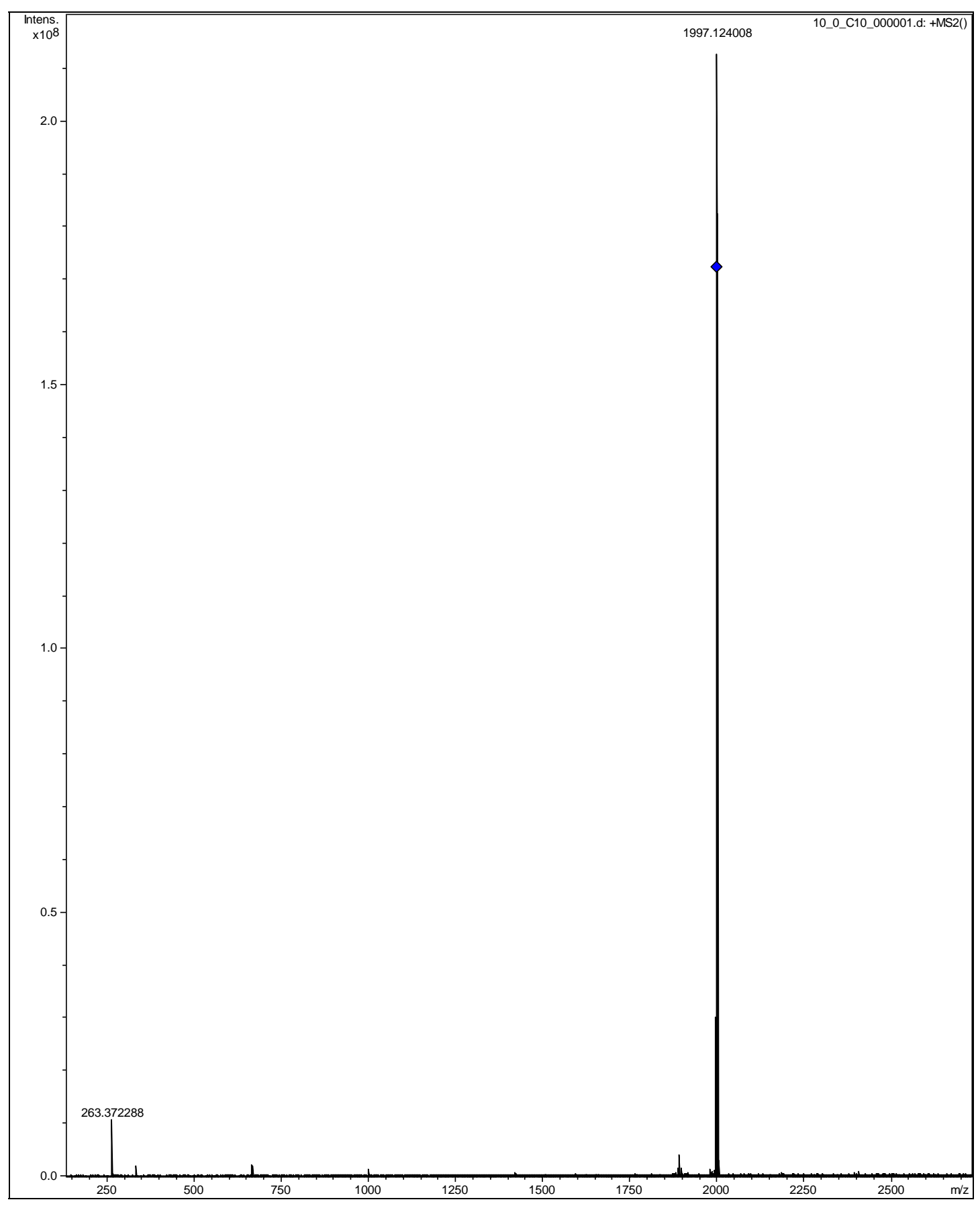

HRMS for 5. 


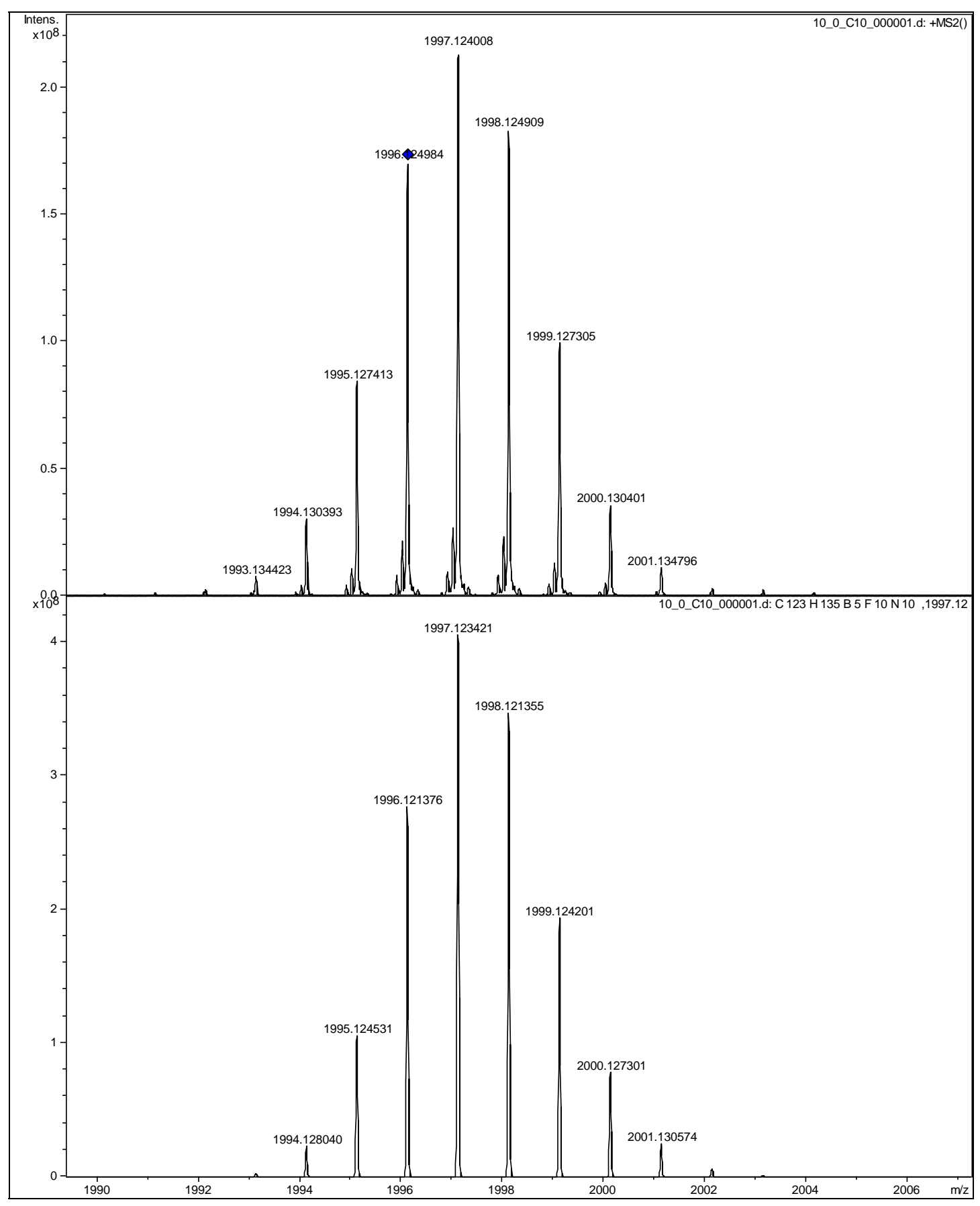

Observed (top) and calculated (bottom) HRMS for 5. 


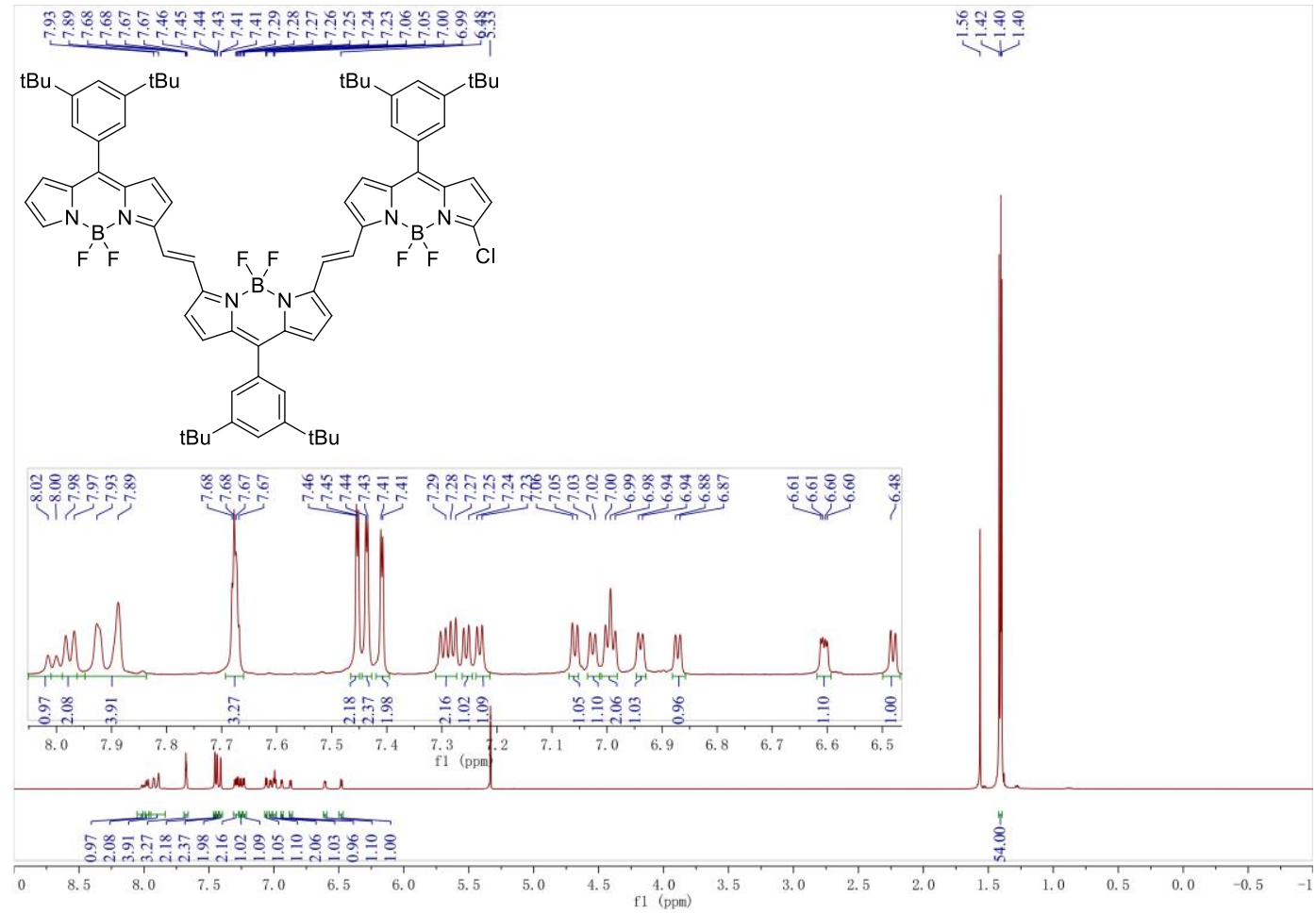

${ }^{1} \mathrm{H}$ NMR (500 MHz) spectrum of $\mathbf{3 C l}$ in $\mathrm{CD}_{2} \mathrm{Cl}_{2}$.

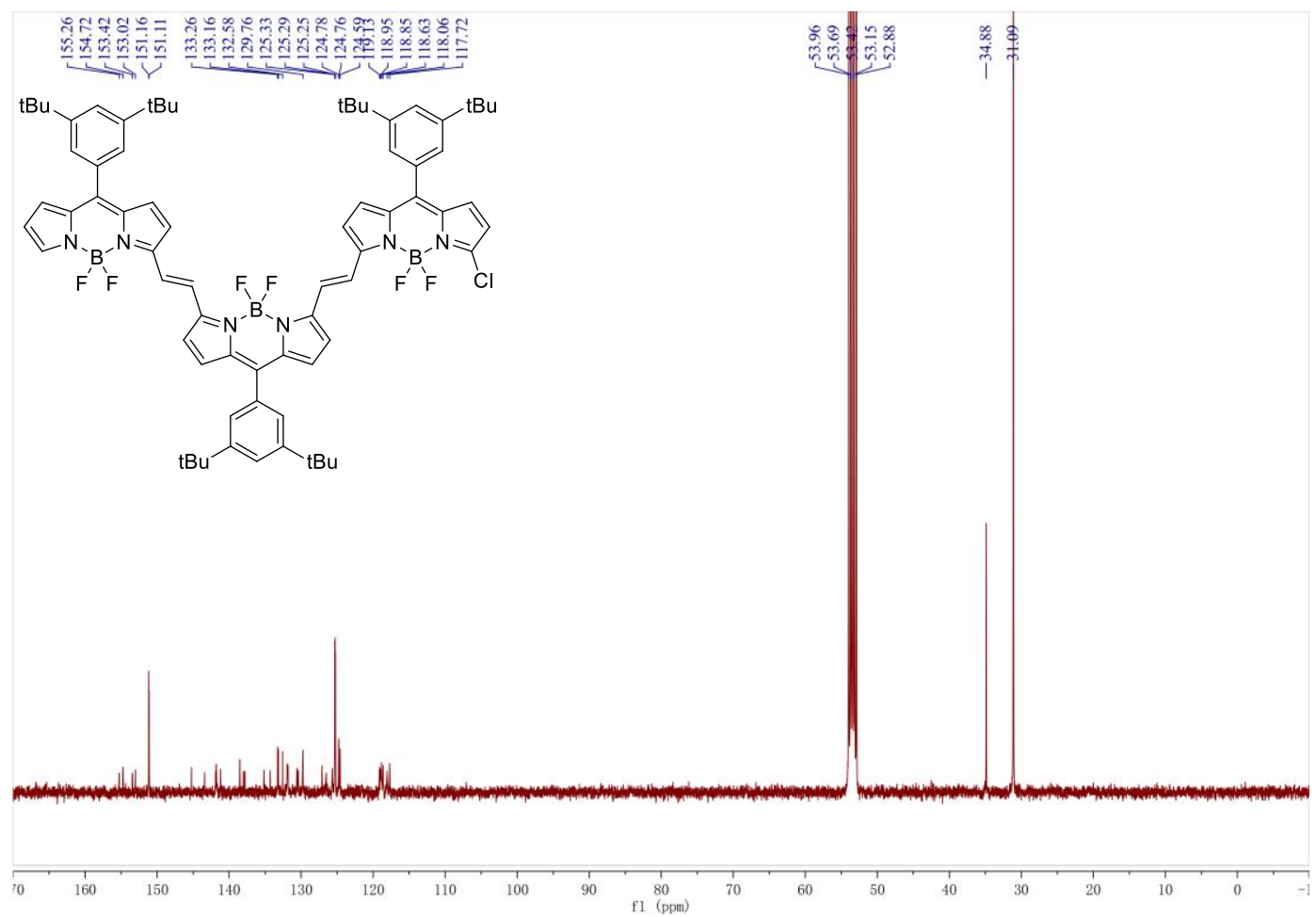

${ }^{13} \mathrm{C}$ NMR (400 MHz) spectrum of $\mathbf{3 C l}$ in $\mathrm{CD}_{2} \mathrm{Cl}_{2}$. 


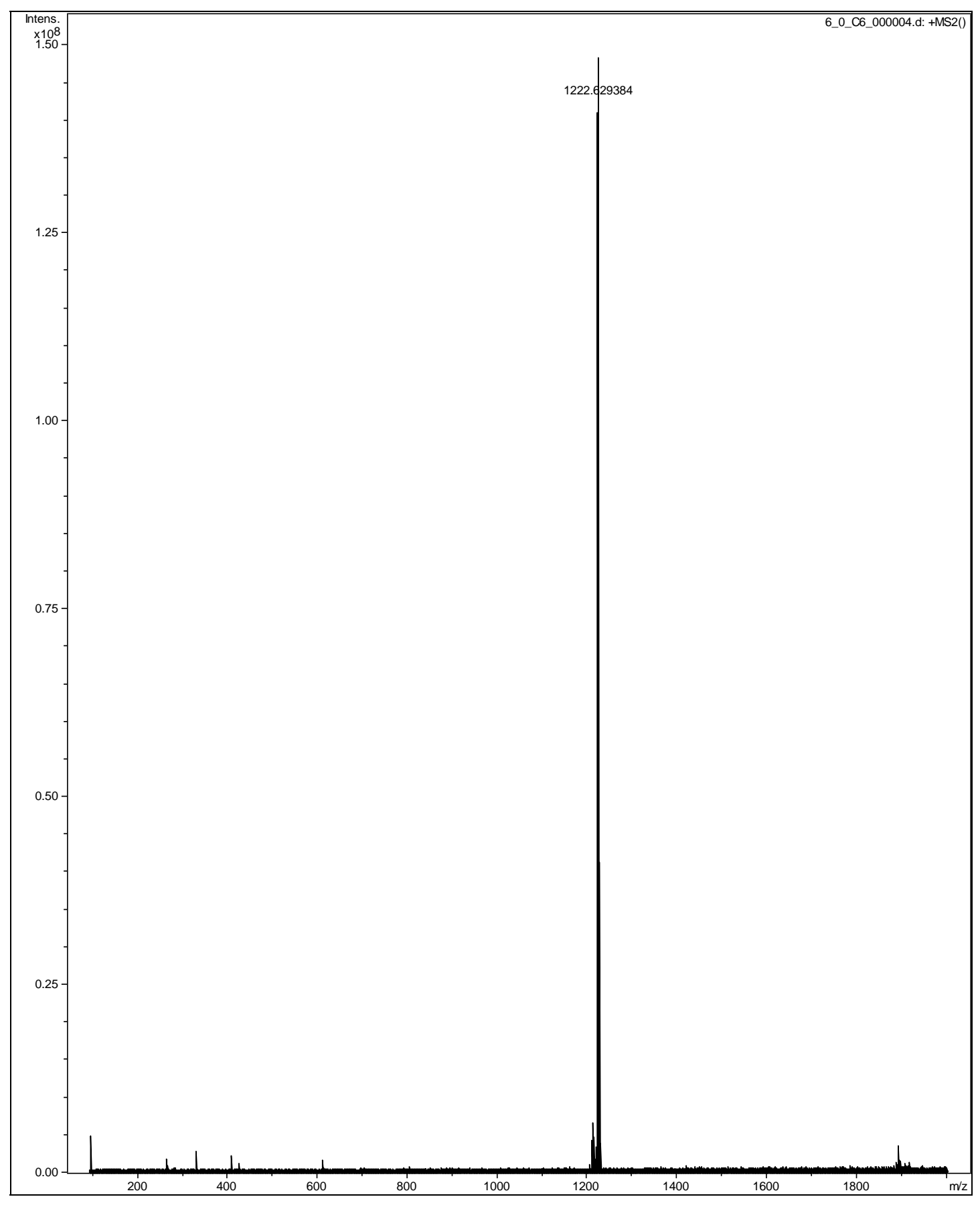

HRMS for $\mathbf{3 C l}$. 


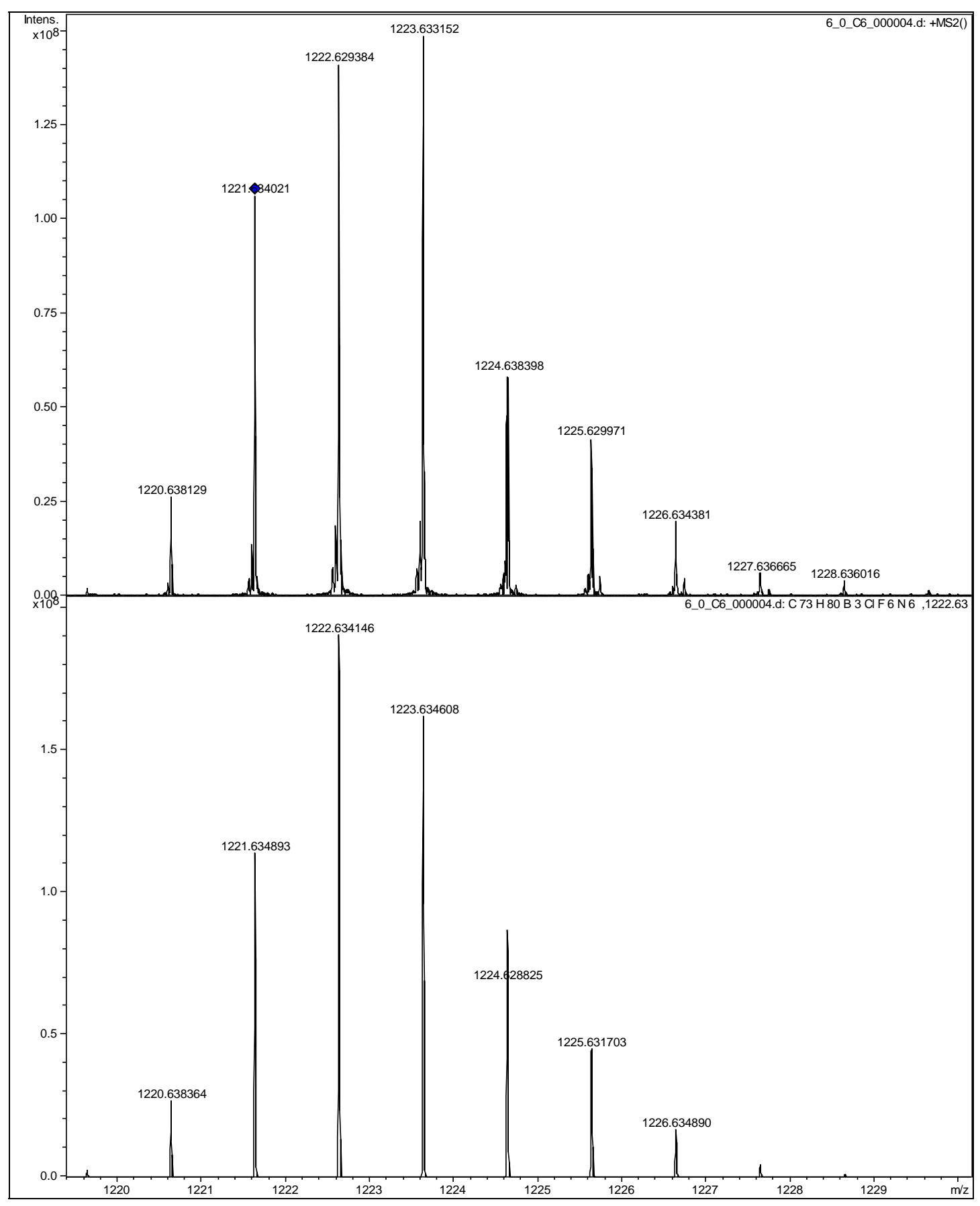

Observed (top) and calculated (bottom) HRMS for $\mathbf{3 C l}$. 


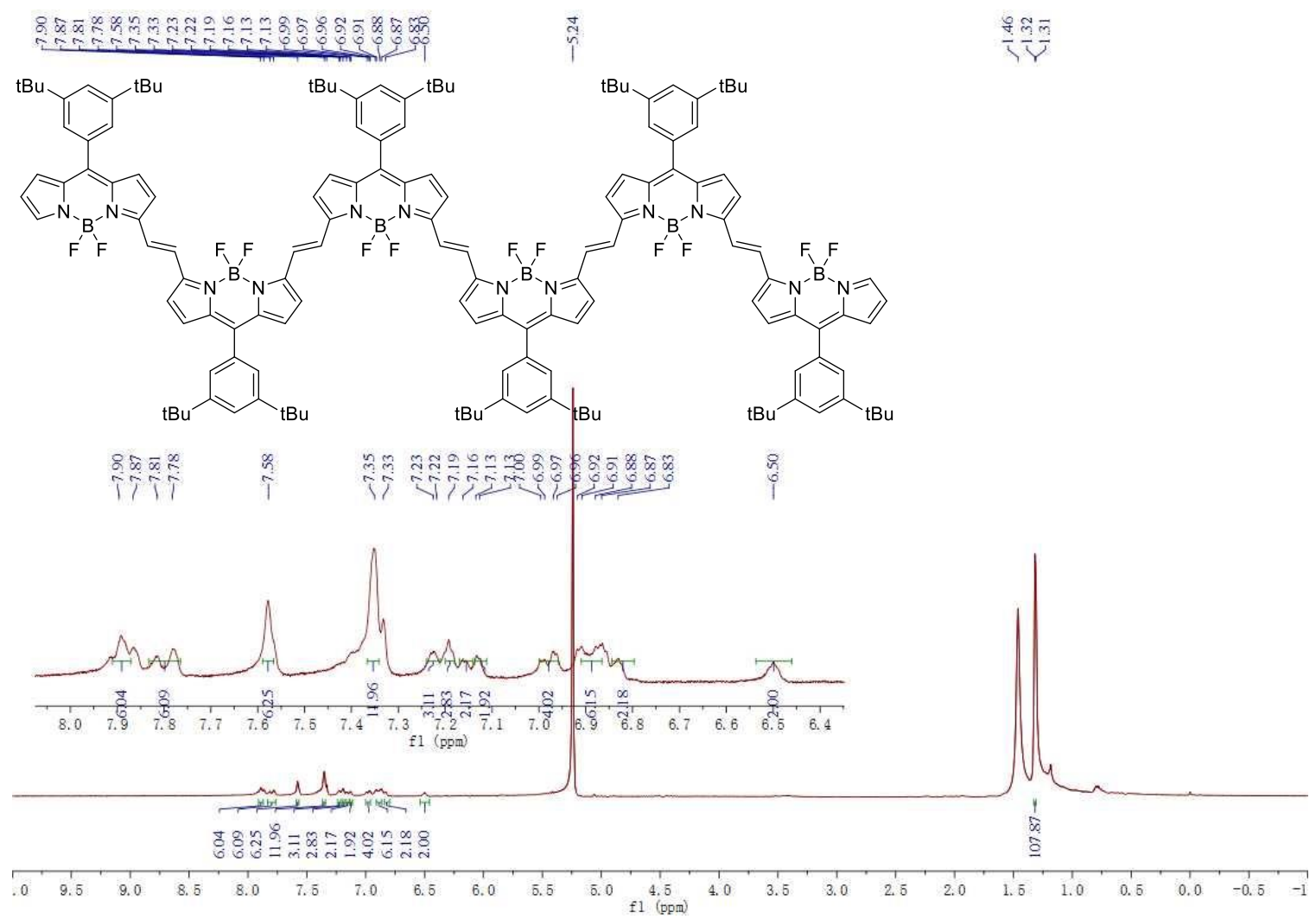

${ }^{1} \mathrm{H}$ NMR (500 MHz) spectrum of 6 in $\mathrm{CD}_{2} \mathrm{Cl}_{2}$. 


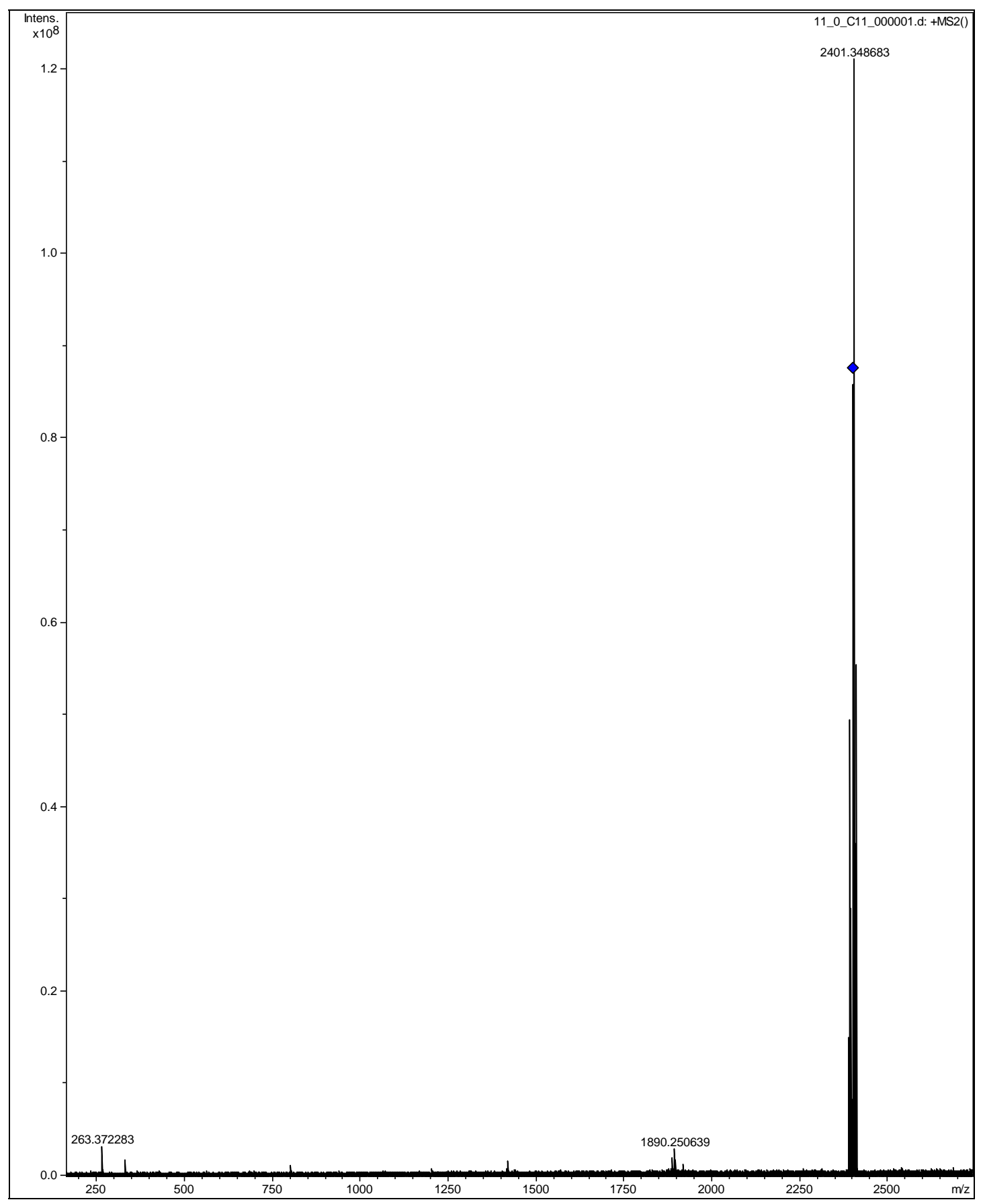

HRMS for 6. 


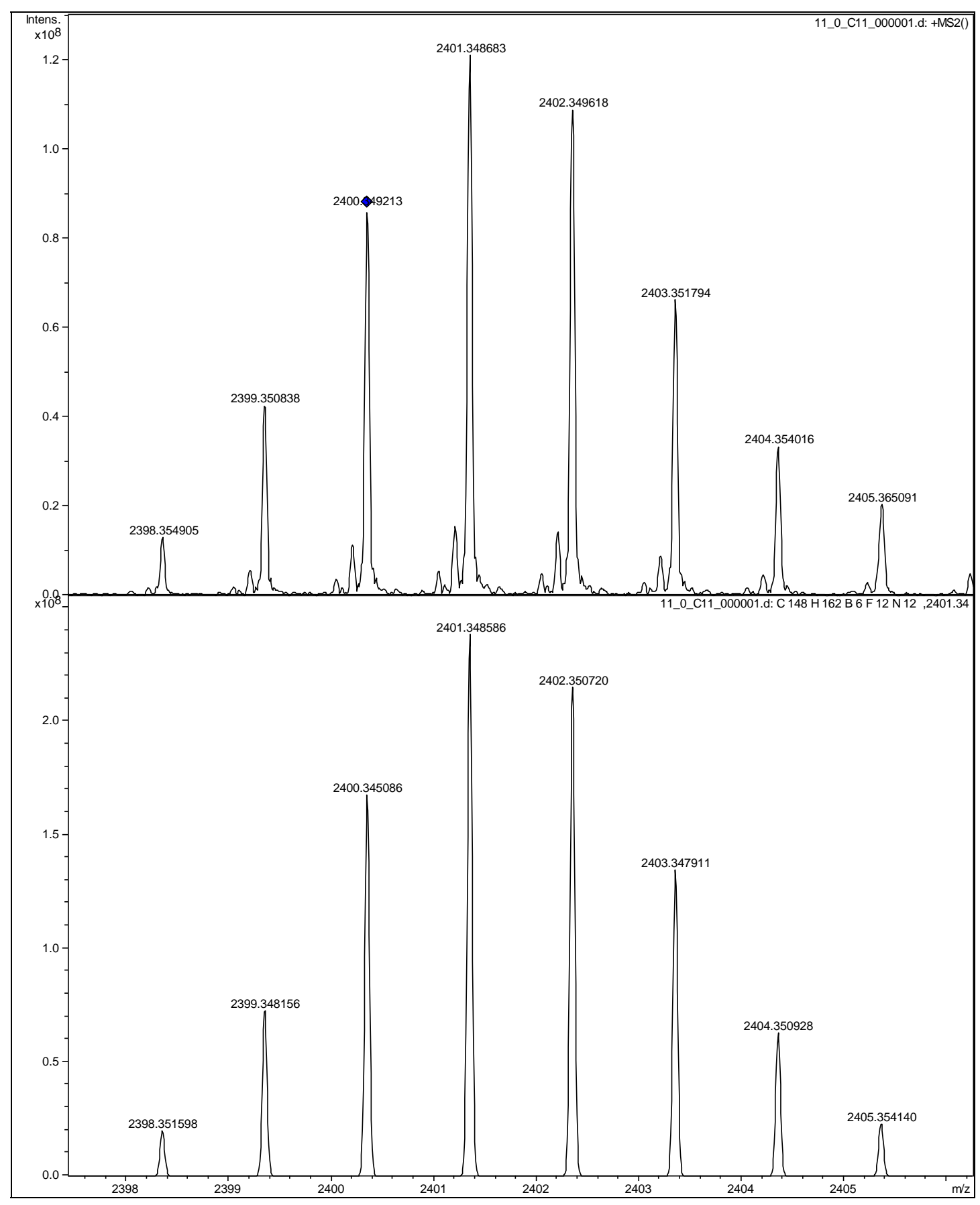

Observed (top) and calculated (bottom) HRMS for 6. 


\section{Optimized Geometries of the Compounds}

\begin{tabular}{crrr}
$\alpha-\beta$ dimer, optimized $S_{0}$ state Geometry. & & \\
$\mathrm{B}$ & 0.00000000 & 0.00000000 & 0.00000000 \\
$\mathrm{C}$ & 0.00000000 & 0.00000000 & 2.60111763 \\
$\mathrm{C}$ & 0.91308790 & 0.00000000 & 3.69438439 \\
$\mathrm{C}$ & 2.19021148 & 0.05910812 & 3.11579097 \\
$\mathrm{C}$ & 2.01813357 & 0.09443535 & 1.71708927 \\
$\mathrm{C}$ & 2.94224274 & 0.19246559 & 0.67636456 \\
$\mathrm{C}$ & 2.54783013 & 0.26743377 & -0.65381412 \\
$\mathrm{C}$ & 3.30346946 & 0.42194387 & -1.84796806 \\
$\mathrm{C}$ & 2.39856854 & 0.47896930 & -2.89660688 \\
$\mathrm{C}$ & 1.10989381 & 0.35602497 & -2.32726184 \\
$\mathrm{~F}$ & -0.55380963 & -1.24647570 & -0.21197305 \\
$\mathrm{~F}$ & -0.93031389 & 1.00886810 & -0.13303835 \\
$\mathrm{~N}$ & 0.64760265 & 0.05306829 & 1.43739330 \\
$\mathrm{~N}$ & 1.19548325 & 0.22971940 & -0.99748578 \\
$\mathrm{H}$ & 3.14211637 & 0.08703622 & 3.62921798 \\
$\mathrm{H}$ & 4.38232960 & 0.48891579 & -1.89949323 \\
$\mathrm{H}$ & 2.61389991 & 0.59798156 & -3.94940685 \\
$\mathrm{H}$ & 0.14583799 & 0.35339723 & -2.81861381 \\
$\mathrm{H}$ & -1.08165823 & -0.03853370 & 2.63167267 \\
$\mathrm{H}$ & 4.00060513 & 0.22872224 & 0.91611602 \\
$\mathrm{~B}$ & 3.36126765 & 0.35492821 & 8.46174242 \\
$\mathrm{C}$ & 5.22077751 & 0.15115542 & 10.26896339 \\
$\mathrm{C}$ & 5.33414819 & 0.03231620 & 11.66870700 \\
$\mathrm{C}$ & 4.03949760 & -0.06383562 & 12.16848618 \\
$\mathrm{C}$ & 3.16160384 & -0.00621459 & 11.05765264 \\
$\mathrm{C}$ & 1.77315100 & -0.11505745 & 10.96316142 \\
$\mathrm{C}$ & 1.12412018 & -0.13109447 & 9.73566677 \\
$\mathrm{C}$ & -0.25554766 & -0.29801303 & 9.42320842 \\
$\mathrm{C}$ & -0.36648874 & -0.29073117 & 8.05174100 \\
$\mathrm{C}$ & 0.94917857 & -0.11576658 & 7.51496128 \\
$\mathrm{~F}$ & 3.49163603 & 1.68490751 & 8.10234889 \\
$\mathrm{~F}$ & 4.02330055 & -0.47408076 & 7.56607576 \\
$\mathrm{~N}$ & 3.92821288 & 0.12878769 & 9.90339742 \\
$\mathrm{~N}$ & 1.83171665 & -0.02311336 & 8.54248276 \\
$\mathrm{H}$ & 6.25932767 & 0.01484660 & 12.22818905 \\
$\mathrm{H}$ & 3.73608991 & -0.17766051 & 13.20105692 \\
$\mathrm{H}$ & -1.04769072 & -0.42441466 & 10.14990066 \\
$\mathrm{H}$ & 5.99987397 & 0.25238318 & 9.52537301 \\
$\mathrm{H}$ & 1.18580920 & -0.21641095 & 11.87054654 \\
$\mathrm{H}$ & -1.26879527 & -0.41608913 & 7.46956456 \\
$\mathrm{C}$ & 1.36945230 & -0.06271141 & 6.14008174 \\
$\mathrm{C}$ & 0.51938000 & -0.04581409 & 5.08324159 \\
$\mathrm{H}$ & -0.55409943 & -0.06440264 & 5.26440535 \\
$\mathrm{H}$ & 2.44128321 & -0.04630762 & 5.97459079 \\
$\mathrm{~S} \mathrm{~F}$ & & & \\
\hline
\end{tabular}

SCF done: -1438.97563682 Hartree No imaginary Frequency.

$\begin{array}{crrr}\boldsymbol{\alpha}-\boldsymbol{m} \text { dimer, optimized } \mathrm{S}_{0} \text { state Geometry. } & \\ \mathrm{B} & -4.78087000 & -0.93700800 & 0.30877000 \\ \mathrm{C} & -5.55872900 & 1.53793000 & 0.22471000 \\ \mathrm{C} & -4.99278400 & 2.82592500 & 0.17009500 \\ \mathrm{C} & -3.62145000 & 2.65182500 & 0.02006900 \\ \mathrm{C} & -3.37866900 & 1.25402300 & -0.02771500\end{array}$




$\begin{array}{lrrr}\mathrm{C} & -2.17854000 & 0.52508300 & -0.20654500 \\ \mathrm{C} & -2.22662400 & -0.87867000 & -0.35217800 \\ \mathrm{C} & -1.25112000 & -1.82616800 & -0.77999500 \\ \mathrm{C} & -1.88529500 & -3.05862000 & -0.85355800 \\ \mathrm{C} & -3.22945400 & -2.84920500 & -0.48671400 \\ \mathrm{~F} & -4.96207200 & -1.21224800 & 1.65242400 \\ \mathrm{~F} & -5.83131400 & -1.40450200 & -0.45681700 \\ \mathrm{~N} & -4.60034700 & 0.60531600 & 0.10524300 \\ \mathrm{~N} & -3.43431000 & -1.55744300 & -0.19353200 \\ \mathrm{H} & -5.53441900 & 3.76004600 & 0.22692300 \\ \mathrm{H} & -2.87680800 & 3.43154300 & -0.06172800 \\ \mathrm{H} & -0.22025000 & -1.62070900 & -1.03159000 \\ \mathrm{H} & -1.44935100 & -4.00084200 & -1.15533500 \\ \mathrm{H} & -4.04598500 & -3.55664300 & -0.43146800 \\ \mathrm{H} & -6.59302900 & 1.24610600 & 0.34775500 \\ \mathrm{~B} & 2.94497700 & -0.65705800 & 0.16785600 \\ \mathrm{C} & 5.14916600 & -2.01580200 & 0.37429700 \\ \mathrm{C} & 6.54554900 & -1.80852200 & 0.32330500 \\ \mathrm{C} & 6.73975300 & -0.45142800 & 0.11015200 \\ \mathrm{C} & 5.45343900 & 0.14661300 & 0.03683300 \\ \mathrm{C} & 5.05980500 & 1.46865800 & -0.13953700 \\ \mathrm{C} & 3.71640900 & 1.83943300 & -0.14339300 \\ \mathrm{C} & 3.10583300 & 3.11046400 & -0.33050400 \\ \mathrm{C} & 1.74072400 & 2.91794100 & -0.27415600 \\ \mathrm{C} & 1.51774100 & 1.52820200 & -0.04327900 \\ \mathrm{~F} & 2.40084800 & -1.29703700 & -0.93859300 \\ \mathrm{~F} & 2.38342200 & -1.13057000 & 1.34053600 \\ \mathrm{~N} & 4.49829800 & -0.85643300 & 0.20213000 \\ \mathrm{~N} & 2.71610000 & 0.89661200 & 0.04101200 \\ \mathrm{H} & 7.30074800 & -2.57426300 & 0.43366100 \\ \mathrm{H} & 7.67996600 & 0.07660300 & 0.01952800 \\ \mathrm{H} & 3.63606900 & 4.04176600 & -0.48110400 \\ \mathrm{H} & 4.60179700 & -2.93689300 & 0.52446400 \\ \mathrm{H} & 5.81754100 & 2.23389300 & -0.27665000 \\ \mathrm{C} & -0.92859600 & 1.26958200 & -0.28666400 \\ \mathrm{H} & 0.96986700 & 3.67142600 & -0.35684100 \\ \mathrm{C} & 0.28200700 & 0.79442600 & 0.08801300 \\ \mathrm{H} & -1.00355400 & 2.28872000 & -0.65674000 \\ \mathrm{H} & 0.36574600 & -0.19770900 & 0.51467500 \\ \mathrm{SCF} & & \\ \mathrm{H} & -1900\end{array}$

SCF done: -1438.97082797 Hartree

No imaginary Frequency.

$\begin{array}{ccrc}\boldsymbol{\beta}-\boldsymbol{\beta} \text { dimer, optimized } & \mathrm{S}_{0} \text { state Geometry. } & \\ \mathrm{B} & -5.13882100 & 1.22882700 & 0.06363500 \\ \mathrm{C} & -2.68254100 & 0.37814200 & 0.03096700 \\ \mathrm{C} & -1.94383700 & -0.84370000 & 0.05495500 \\ \mathrm{C} & -2.90622700 & -1.85783000 & 0.06526600 \\ \mathrm{C} & -4.18065800 & -1.24500100 & 0.04439800 \\ \mathrm{C} & -5.46635600 & -1.77624500 & 0.01244700 \\ \mathrm{C} & -6.59915800 & -0.96844100 & -0.05079700 \\ \mathrm{C} & -7.97772600 & -1.29535600 & -0.13440100 \\ \mathrm{C} & -8.67611500 & -0.09745900 & -0.20598100 \\ \mathrm{C} & -7.71436200 & 0.93507400 & -0.16295200 \\ \mathrm{~F} & -5.10410600 & 1.88916400 & 1.27534800 \\ \mathrm{~F} & -4.99266600 & 2.09383800 & -1.00050600 \\ \mathrm{~N} & -3.99330700 & 0.14084000 & 0.02615400\end{array}$




$\begin{array}{lrrr}\mathrm{N} & -6.47950200 & 0.42065400 & -0.07053500 \\ \mathrm{H} & -2.72308700 & -2.92474700 & 0.07781400 \\ \mathrm{H} & -8.38225200 & -2.29908200 & -0.14556300 \\ \mathrm{H} & -9.74581900 & 0.03942500 & -0.28350200 \\ \mathrm{H} & -7.86228100 & 2.00643900 & -0.19354300 \\ \mathrm{H} & -2.31256500 & 1.39481500 & 0.02065900 \\ \mathrm{H} & -5.58989100 & -2.85505100 & 0.02284600 \\ \mathrm{~B} & 5.35389700 & 1.30493100 & 0.01959100 \\ \mathrm{C} & 7.84222200 & 0.56665400 & -0.18297300 \\ \mathrm{C} & 8.61499700 & -0.61636300 & -0.17939200 \\ \mathrm{C} & 7.72345500 & -1.67346600 & -0.06744600 \\ \mathrm{C} & 6.41953900 & -1.11316500 & -0.00694000 \\ \mathrm{C} & 5.16649200 & -1.71218000 & 0.07414800 \\ \mathrm{C} & 3.98920300 & -0.96677000 & 0.07168500 \\ \mathrm{C} & 2.63238600 & -1.35965800 & 0.09731800 \\ \mathrm{C} & 1.85430200 & -0.19634700 & 0.03925700 \\ \mathrm{C} & 2.78736600 & 0.87870100 & -0.01766600 \\ \mathrm{~F} & 5.43215700 & 1.99544200 & 1.21241100 \\ \mathrm{~F} & 5.36716100 & 2.15116100 & -1.06926900 \\ \mathrm{~N} & 6.53890800 & 0.27461100 & -0.08015100 \\ \mathrm{~N} & 4.04252000 & 0.42824600 & 0.00201900 \\ \mathrm{H} & 9.69243400 & -0.66615400 & -0.25451800 \\ \mathrm{H} & 7.95076100 & -2.73112600 & -0.03797000 \\ \mathrm{H} & 2.28404300 & -2.38295500 & 0.14595700 \\ \mathrm{H} & 2.58143900 & 1.94041800 & -0.07027200 \\ \mathrm{H} & 8.17084300 & 1.59513100 & -0.25346800 \\ \mathrm{H} & 5.10309200 & -2.79503800 & 0.12488400 \\ \mathrm{C} & -0.50462000 & -1.01168000 & 0.06276200 \\ \mathrm{C} & 0.41505600 & -0.02093000 & 0.02860900 \\ \mathrm{H} & -0.16155800 & -2.04487100 & 0.09854800 \\ \mathrm{H} & 0.07572500 & 1.01323700 & -0.01186300 \\ \mathrm{SCF} & & & \end{array}$

SCF done: -1438.96814638 Hartree

No imaginary Frequency.

$\boldsymbol{\beta}-\mathbf{m}$ dimer, optimized $\mathrm{S}_{0}$ state Geometry.

$\begin{array}{lrrr}\text { B } & -5.57725000 & -0.06686800 & 0.38440700 \\ \mathrm{C} & -5.20053500 & 2.50049300 & 0.35597800 \\ \mathrm{C} & -4.12867700 & 3.41333700 & 0.31811400 \\ \mathrm{C} & -2.97339400 & 2.66320700 & 0.13138800 \\ \mathrm{C} & -3.36669900 & 1.30198300 & 0.04137600 \\ \mathrm{C} & -2.61165600 & 0.12828700 & -0.18764100 \\ \mathrm{C} & -3.28494800 & -1.10098900 & -0.38743700 \\ \mathrm{C} & -2.83823800 & -2.34531500 & -0.90708700 \\ \mathrm{C} & -3.95061500 & -3.17411500 & -1.00805600 \\ \mathrm{C} & -5.05428000 & -2.42335300 & -0.56331900 \\ \mathrm{~F} & -5.79669600 & -0.28281000 & 1.73369100 \\ \mathrm{~F} & -6.76040600 & -0.00153700 & -0.32485200 \\ \mathrm{~N} & -4.74885400 & 1.24813800 & 0.18800700 \\ \mathrm{~N} & -4.66107100 & -1.19216700 & -0.20117000 \\ \mathrm{H} & -1.96410900 & 3.04234600 & 0.04861500 \\ \mathrm{H} & -1.83079700 & -2.57118900 & -1.22473100 \\ \mathrm{H} & -3.98220000 & -4.18788600 & -1.38291900 \\ \mathrm{H} & -6.09712400 & -2.70326300 & -0.50010700 \\ \mathrm{H} & -6.25639200 & 2.68489800 & 0.50169600 \\ \mathrm{~B} & 4.46366000 & 1.03541600 & -0.65127700 \\ \mathrm{C} & 6.99481500 & 0.80941600 & -0.09343900\end{array}$




$\begin{array}{lrrr}\mathrm{C} & 7.88710600 & -0.11446700 & 0.49988200 \\ \mathrm{C} & 7.12076400 & -1.18299600 & 0.93646100 \\ \mathrm{C} & 5.76936800 & -0.89008700 & 0.60371200 \\ \mathrm{C} & 4.59481800 & -1.59030900 & 0.84423600 \\ \mathrm{C} & 3.34506900 & -1.08477400 & 0.48260600 \\ \mathrm{C} & 2.04014900 & -1.58122500 & 0.67905900 \\ \mathrm{C} & 1.14426900 & -0.63645800 & 0.16017600 \\ \mathrm{C} & 1.95590100 & 0.41939200 & -0.35008100 \\ \mathrm{~F} & 4.33071900 & 2.31977400 & -0.17118600 \\ \mathrm{~F} & 4.52971300 & 0.99674600 & -2.02828800 \\ \mathrm{~N} & 5.73879700 & 0.35189800 & -0.03387100 \\ \mathrm{~N} & 3.24706600 & 0.15365400 & -0.15999800 \\ \mathrm{H} & 8.95713400 & 0.01145200 & 0.58923200 \\ \mathrm{H} & 7.45876200 & -2.07631900 & 1.44514700 \\ \mathrm{H} & 1.78703400 & -2.51778800 & 1.15926100 \\ \mathrm{H} & 1.65295900 & 1.33417300 & -0.84186500 \\ \mathrm{H} & 7.21061400 & 1.76564200 & -0.55167900 \\ \mathrm{H} & 4.64700800 & -2.55133300 & 1.34694900 \\ \mathrm{H} & -4.20649000 & 4.48792500 & 0.40949900 \\ \mathrm{C} & -0.29850900 & -0.73274700 & 0.16102100 \\ \mathrm{H} & -0.70409500 & -1.65976600 & 0.55785400 \\ \mathrm{C} & -1.16156300 & 0.23391400 & -0.23650400 \\ \mathrm{H} & -0.76475700 & 1.19898500 & -0.54032300\end{array}$

SCF done: -1438.96560592 Hartree

No imaginary Frequency.

$\begin{array}{crrr}\text { m-m dimer, optimized } & \text { S state Geometry. } & \\ \text { B } & -4.84715400 & -0.29408100 & 0.30515200 \\ \text { C } & -4.57361300 & 2.03900100 & 1.41279600 \\ \text { C } & -3.54204800 & 2.93351000 & 1.76846100 \\ \text { C } & -2.36375000 & 2.40422400 & 1.26111000 \\ \text { C } & -2.70226200 & 1.19724900 & 0.58921100 \\ \text { C } & -1.90795800 & 0.28269700 & -0.13148100 \\ \text { C } & -2.51726500 & -0.78589200 & -0.82283500 \\ \text { C } & -2.01312400 & -1.68586100 & -1.80248700 \\ \text { C } & -3.08161400 & -2.45760700 & -2.23985700 \\ \text { C } & -4.21730000 & -2.01291700 & -1.53345800 \\ \text { F } & -5.04749500 & -1.08139000 & 1.42326700 \\ \text { F } & -6.03220500 & 0.02427300 & -0.32456300 \\ \text { N } & -4.07581200 & 1.01059100 & 0.71168800 \\ \text { N } & -3.88430400 & -1.01895200 & -0.69746100 \\ \text { H } & -1.37376700 & 2.83150400 & 1.34291700 \\ \text { H } & -0.99780700 & -1.71423500 & -2.17055200 \\ \text { H } & -3.06713300 & -3.23263400 & -2.99355000 \\ \text { H } & -5.24225400 & -2.35293500 & -1.59668100 \\ \text { H } & -5.63160800 & 2.08986700 & 1.63284400 \\ \text { B } & 4.84716400 & 0.29404900 & 0.30515200 \\ \text { C } & 4.57356900 & -2.03901500 & 1.41281900 \\ \text { C } & 3.54198700 & -2.93351500 & 1.76846100 \\ \text { C } & 2.36371000 & -2.40423100 & 1.26105900 \\ \text { C } & 2.70224000 & -1.19723000 & 0.58921900 \\ \text { C } & 1.90795200 & -0.28264800 & -0.13145000 \\ \text { C } & 2.51728000 & 0.78592200 & -0.82281400 \\ \text { C } & 2.01316700 & 1.68586400 & -1.80250600 \\ \text { C } & 3.08165500 & 2.45764600 & -2.23981700 \\ \text { C } & 4.21733200 & 2.01293700 & -1.53341500\end{array}$




$\begin{array}{crrr}\mathrm{F} & 5.04751500 & 1.08134600 & 1.42327400 \\ \mathrm{~F} & 6.03221100 & -0.02432300 & -0.32456100 \\ \mathrm{~N} & 4.07579700 & -1.01061100 & 0.71168200 \\ \mathrm{~N} & 3.88432700 & 1.01894400 & -0.69745500 \\ \mathrm{H} & 3.66423500 & -3.85365700 & 2.32274600 \\ \mathrm{H} & 1.37372200 & -2.83150700 & 1.34283200 \\ \mathrm{H} & 0.99786000 & 1.71423500 & -2.17059800 \\ \mathrm{H} & 5.24228700 & 2.35295700 & -1.59661600 \\ \mathrm{H} & 5.63155800 & -2.08989000 & 1.63289100 \\ \mathrm{H} & 3.06718300 & 3.23269400 & -2.99348800 \\ \mathrm{H} & -3.66431800 & 3.85365300 & 2.32274000 \\ \mathrm{C} & 0.46073100 & -0.49452500 & -0.16176500 \\ \mathrm{H} & 0.12122700 & -1.52305600 & -0.08993300 \\ \mathrm{C} & -0.46073800 & 0.49457300 & -0.16176700 \\ \mathrm{H} & -0.12123600 & 1.52310200 & -0.08991400\end{array}$

SCF done: -1438.95826719 Hartree

No imaginary Frequency.

Trimer 3, optimized $\mathrm{S}_{0}$ state Geometry.

$\begin{array}{lrrr}\text { B } & 7.19372200 & -0.36377200 & 0.29774300 \\ \mathrm{C} & 9.73574000 & -0.87174400 & 0.07856500 \\ \mathrm{C} & 10.59342400 & -1.98758000 & -0.02296100 \\ \mathrm{C} & 9.78022200 & -3.11255000 & -0.08691900 \\ \mathrm{C} & 8.43766400 & -2.65742200 & -0.02821400 \\ \mathrm{C} & 7.22266900 & -3.33523600 & -0.11146400 \\ \mathrm{C} & 6.00772700 & -2.65721500 & -0.13401100 \\ \mathrm{C} & 4.67925000 & -3.15156700 & -0.28128300 \\ \mathrm{C} & 3.83842000 & -2.06278100 & -0.29317000 \\ \mathrm{C} & 4.65104800 & -0.89310000 & -0.15104300 \\ \mathrm{~F} & 7.11802600 & 0.02657400 & 1.62201800 \\ \mathrm{~F} & 7.25422800 & 0.73372500 & -0.54943100 \\ \mathrm{~N} & 8.45370100 & -1.26854000 & 0.07594900 \\ \mathrm{~N} & 5.95404500 & -1.27246900 & -0.05691100 \\ \mathrm{H} & 11.67378900 & -1.95267700 & -0.05052600 \\ \mathrm{H} & 10.08686600 & -4.14618100 & -0.18020200 \\ \mathrm{H} & 4.41084400 & -4.19533500 & -0.38074000 \\ \mathrm{H} & 2.76335900 & -2.06609100 & -0.40641100 \\ \mathrm{H} & 9.98514100 & 0.17814900 & 0.15439600 \\ \mathrm{~B} & 0.00003100 & 1.75887500 & 0.17338300 \\ \mathrm{C} & 2.56337500 & 2.27715100 & -0.15064100 \\ \mathrm{C} & 3.38507300 & 3.44537900 & -0.22226800 \\ \mathrm{C} & 2.54974800 & 4.53910900 & -0.21166900 \\ \mathrm{C} & 1.21472400 & 4.04800900 & -0.13668900 \\ \mathrm{C} & -0.00000200 & 4.72858700 & -0.13922300 \\ \mathrm{C} & -1.21471800 & 4.04799400 & -0.13655600 \\ \mathrm{C} & -2.54974800 & 4.53908800 & -0.21148200 \\ \mathrm{C} & -3.38506700 & 3.44535500 & -0.22203800 \\ \mathrm{C} & -2.56335700 & 2.27712800 & -0.15047200 \\ \mathrm{~F} & -0.00002600 & 0.66602900 & -0.69450900 \\ \mathrm{~F} & 0.00012500 & 1.34053800 & 1.49127200 \\ \mathrm{~N} & 1.25629100 & 2.66324300 & -0.10201500 \\ \mathrm{~N} & -1.25627500 & 2.66322700 & -0.10187700 \\ \mathrm{H} & 4.46413100 & 3.44633200 & -0.28787700 \\ \mathrm{H} & 2.82708000 & 5.58372400 & -0.26621100 \\ \mathrm{H} & -2.82707800 & 5.58370600 & -0.26599700 \\ \mathrm{H} & -4.46412900 & 3.44629900 & -0.28757000 \\ & & & \\ & & & \\ & & \end{array}$




$\begin{array}{lrrr}\mathrm{C} & 4.25501600 & 0.48517000 & -0.13060300 \\ \mathrm{C} & 2.95795400 & 0.89915600 & -0.14909400 \\ \mathrm{H} & 5.06223300 & 1.20891700 & -0.11179900 \\ \mathrm{H} & 2.15241900 & 0.17407100 & -0.17500000 \\ \mathrm{~B} & -7.19376300 & -0.36375200 & 0.29769400 \\ \mathrm{C} & -4.65104200 & -0.89310900 & -0.15083500 \\ \mathrm{C} & -3.83840800 & -2.06279200 & -0.29288900 \\ \mathrm{C} & -4.67923700 & -3.15157900 & -0.28099200 \\ \mathrm{C} & -6.00772300 & -2.65721400 & -0.13382200 \\ \mathrm{C} & -7.22266500 & -3.33523700 & -0.11132700 \\ \mathrm{C} & -8.43766700 & -2.65742000 & -0.02821000 \\ \mathrm{C} & -9.78021800 & -3.11255600 & -0.08701700 \\ \mathrm{C} & -10.59343200 & -1.98758600 & -0.02321900 \\ \mathrm{C} & -9.73576200 & -0.87173500 & 0.07826600 \\ \mathrm{~F} & -7.11819500 & 0.02668400 & 1.62195700 \\ \mathrm{~F} & -7.25419500 & 0.73368400 & -0.54955700 \\ \mathrm{~N} & -5.95404400 & -1.27246700 & -0.05676900 \\ \mathrm{~N} & -8.45372100 & -1.26853000 & 0.07584600 \\ \mathrm{H} & -2.76334000 & -2.06610900 & -0.40606600 \\ \mathrm{H} & -4.41082100 & -4.19535000 & -0.38037600 \\ \mathrm{H} & -10.08685200 & -4.14619700 & -0.18023900 \\ \mathrm{H} & -11.67379400 & -1.95269000 & -0.05087800 \\ \mathrm{H} & -9.98517100 & 0.17816500 & 0.15397000 \\ \mathrm{C} & -2.95794300 & 0.89913900 & -0.14890000 \\ \mathrm{C} & -4.25500700 & 0.48516000 & -0.13040600 \\ \mathrm{H} & -2.15241000 & 0.17405100 & -0.17478100 \\ \mathrm{H} & -5.06221400 & 1.20891700 & -0.11163300 \\ \mathrm{H} & 7.22221600 & -4.41802200 & -0.18893800 \\ \mathrm{H} & -0.00000900 & 5.81323800 & -0.17027000 \\ \mathrm{H} & -7.22220000 & -4.41802800 & -0.18873800 \\ \mathrm{SCF}\end{array}$

SCF done: -2196.58703706 Hartree

No imaginary Frequency.

Tetramer 4, optimized $\mathrm{S}_{0}$ state Geometry.

$\begin{array}{lrrr}\text { B } & 3.43921900 & -1.50019300 & 0.09494100 \\ \mathrm{C} & 5.93295500 & -2.32331900 & -0.09626800 \\ \mathrm{C} & 6.61185700 & -3.58102900 & -0.14328600 \\ \mathrm{C} & 5.65243000 & -4.56672600 & -0.19039500 \\ \mathrm{C} & 4.38339800 & -3.91962600 & -0.17516000 \\ \mathrm{C} & 3.09738700 & -4.44982700 & -0.23976500 \\ \mathrm{C} & 1.97394300 & -3.62806500 & -0.27949400 \\ \mathrm{C} & 0.59339600 & -3.95532700 & -0.40968500 \\ \mathrm{C} & -0.10423200 & -2.76966900 & -0.43895200 \\ \mathrm{C} & 0.84763800 & -1.70767300 & -0.32540700 \\ \mathrm{~F} & 3.43328300 & -1.07016100 & 1.40970800 \\ \mathrm{~F} & 3.60800400 & -0.42463600 & -0.77731500 \\ \mathrm{~N} & 4.58831100 & -2.55033900 & -0.12073500 \\ \mathrm{~N} & 2.09657500 & -2.24895900 & -0.23173000 \\ \mathrm{H} & 7.68502900 & -3.71031500 & -0.15926300 \\ \mathrm{H} & 5.80555300 & -5.63654500 & -0.24647100 \\ \mathrm{H} & 0.19593800 & -4.95900500 & -0.48589100 \\ \mathrm{H} & -1.17221600 & -2.64096800 & -0.54604800 \\ \mathrm{~B} & -3.43952500 & 1.49239100 & 0.03100900 \\ \mathrm{C} & -0.84355700 & 1.68793700 & -0.36985900 \\ \mathrm{C} & 0.11056500 & 2.74644700 & -0.49692300 \\ \mathrm{C} & -0.58597000 & 3.93305800 & -0.49965000\end{array}$




\begin{tabular}{|c|c|c|c|}
\hline $\mathrm{C}$ & -1.96808600 & 3.60982500 & -0.37607300 \\
\hline $\mathrm{C}$ & -3.09100600 & 4.43311000 & -0.36639300 \\
\hline $\mathrm{C}$ & -4.37823700 & 3.90540700 & -0.30539300 \\
\hline $\mathrm{C}$ & -5.64609100 & 4.55307500 & -0.35533800 \\
\hline $\mathrm{C}$ & -6.60718200 & 3.56952000 & -0.30232900 \\
\hline $\mathrm{C}$ & -5.93069100 & 2.31238400 & -0.21592900 \\
\hline $\mathrm{F}$ & -3.60107000 & 0.39629800 & -0.81670200 \\
\hline $\mathrm{F}$ & -3.44675100 & 1.09485900 & 1.35558000 \\
\hline $\mathrm{N}$ & -2.09276000 & 2.23217700 & -0.30020200 \\
\hline $\mathrm{N}$ & -4.58546000 & 2.53801200 & -0.22207300 \\
\hline $\mathrm{H}$ & 1.17934800 & 2.61456700 & -0.59140000 \\
\hline $\mathrm{H}$ & -0.18671300 & 4.93451300 & -0.59361700 \\
\hline $\mathrm{H}$ & -5.79706200 & 5.62165500 & -0.43563700 \\
\hline $\mathrm{H}$ & -7.67984400 & 3.70050200 & -0.33207300 \\
\hline $\mathrm{C}$ & 0.62112000 & -0.29395400 & -0.32871400 \\
\hline $\mathrm{C}$ & -0.61804800 & 0.27435600 & -0.34025600 \\
\hline $\mathrm{H}$ & 1.50795400 & 0.32959300 & -0.33196500 \\
\hline $\mathrm{H}$ & -1.50509200 & -0.34890000 & -0.33705400 \\
\hline B & -10.84095000 & 0.28257900 & 0.40050400 \\
\hline $\mathrm{C}$ & -8.38608500 & -0.57556700 & -0.02645100 \\
\hline $\mathrm{C}$ & -7.72203900 & -1.84111400 & -0.10800400 \\
\hline $\mathrm{C}$ & -8.68715000 & -2.81796700 & -0.02682600 \\
\hline $\mathrm{C}$ & -9.94436800 & -2.15939300 & 0.10271200 \\
\hline $\mathrm{C}$ & -11.23098800 & -2.68415800 & 0.17669500 \\
\hline $\mathrm{C}$ & -12.35483800 & -1.86169200 & 0.23295700 \\
\hline $\mathrm{C}$ & -13.74285100 & -2.15524000 & 0.21742800 \\
\hline $\mathrm{C}$ & -14.41496300 & -0.93899900 & 0.22358500 \\
\hline $\mathrm{C}$ & -13.42881400 & 0.06985000 & 0.24775900 \\
\hline $\mathrm{F}$ & -10.69728900 & 0.75270500 & 1.69305000 \\
\hline $\mathrm{F}$ & -10.78417500 & 1.31754000 & -0.52167600 \\
\hline $\mathrm{N}$ & -9.72376300 & -0.78923500 & 0.09956900 \\
\hline $\mathrm{N}$ & -12.20333700 & -0.47734200 & 0.25388500 \\
\hline $\mathrm{H}$ & -6.65680400 & -1.98104600 & -0.22745600 \\
\hline $\mathrm{H}$ & -8.54730700 & -3.89044400 & -0.06698100 \\
\hline $\mathrm{H}$ & -14.17187300 & -3.14836600 & 0.19025600 \\
\hline $\mathrm{H}$ & -15.48380400 & -0.77625600 & 0.20698300 \\
\hline C & -6.48891300 & 0.99431600 & -0.14721100 \\
\hline C & -7.82588700 & 0.74322100 & -0.08298300 \\
\hline $\mathrm{H}$ & -5.77918700 & 0.17483900 & -0.15822600 \\
\hline $\mathrm{H}$ & -8.53747000 & 1.56136200 & -0.08446000 \\
\hline $\mathrm{H}$ & 2.96904400 & -5.52642300 & -0.28289000 \\
\hline $\mathrm{H}$ & -2.96115200 & 5.50836900 & -0.43228200 \\
\hline $\mathrm{H}$ & -11.36094200 & -3.76181100 & 0.16291200 \\
\hline B & 10.86648700 & -0.25895900 & -0.07171600 \\
\hline $\mathrm{C}$ & 13.42114100 & -0.04671900 & 0.37025800 \\
\hline $\mathrm{C}$ & 14.39166800 & 0.96258100 & 0.54408200 \\
\hline $\mathrm{C}$ & 13.71436300 & 2.17584900 & 0.52724200 \\
\hline $\mathrm{C}$ & 12.33841500 & 1.87990400 & 0.34788700 \\
\hline $\mathrm{C}$ & 11.20946400 & 2.69665600 & 0.31516700 \\
\hline $\mathrm{C}$ & 9.92609800 & 2.16619700 & 0.22736500 \\
\hline $\mathrm{C}$ & 8.65760200 & 2.81545800 & 0.24576100 \\
\hline $\mathrm{C}$ & 7.69640500 & 1.83329900 & 0.18266100 \\
\hline $\mathrm{C}$ & 8.37452200 & 0.57412200 & 0.12349500 \\
\hline $\mathrm{F}$ & 10.86472200 & -0.67321000 & -1.39154800 \\
\hline $\mathrm{F}$ & 10.71968900 & -1.33314000 & 0.79382000 \\
\hline $\mathrm{N}$ & 12.19984700 & 0.49729300 & 0.25269800 \\
\hline
\end{tabular}




$\begin{array}{lrrc}\mathrm{N} & & & \\ \mathrm{H} & 15.41630200 & 0.79626300 & 0.15270700 \\ \mathrm{H} & 14.13102800 & 3.16789400 & 0.67095500 \\ \mathrm{H} & 8.50644600 & 3.88484000 & 0.31608500 \\ \mathrm{H} & 6.62348400 & 1.96416600 & 0.19879200 \\ \mathrm{H} & 13.55101200 & -1.11967200 & 0.32388600 \\ \mathrm{H} & 11.32982200 & 3.77313300 & 0.38696000 \\ \mathrm{C} & 6.48840300 & -1.00288900 & -0.05118900 \\ \mathrm{C} & 7.82012200 & -0.74759200 & 0.07277500 \\ \mathrm{H} & 5.78048100 & -0.18577200 & -0.13393000 \\ \mathrm{H} & 8.52999600 & -1.56314800 & 0.15654100 \\ \mathrm{H} & -13.55032900 & 1.14468300 & 0.26327500\end{array}$

SCF done: -2954.19301065 Hartree

No imaginary Frequency.

Pentamer 5, optimized $\mathrm{S}_{0}$ state Geometry.

$\begin{array}{lrrr}\text { B } & 0.00000000 & 0.00000000 & 0.00000000 \\ \mathrm{C} & 0.00000000 & 0.00000000 & 2.63501527 \\ \mathrm{C} & 0.98389830 & 0.00000000 & 3.67278490 \\ \mathrm{C} & 2.21597230 & 0.13220137 & 3.07389660 \\ \mathrm{C} & 1.99393223 & 0.21729021 & 1.66934723 \\ \mathrm{C} & 2.89326062 & 0.39623798 & 0.62134694 \\ \mathrm{C} & 2.45754460 & 0.53285645 & -0.69404374 \\ \mathrm{C} & 3.19031283 & 0.79627777 & -1.88725712 \\ \mathrm{C} & 2.27754808 & 0.88873413 & -2.91248222 \\ \mathrm{C} & 0.97796849 & 0.67929281 & -2.35171360 \\ \mathrm{~F} & -0.33450914 & -1.31985136 & -0.24404208 \\ \mathrm{~F} & -1.12081134 & 0.82402153 & -0.10833400 \\ \mathrm{~N} & 0.63085231 & 0.13634001 & 1.43335282 \\ \mathrm{~N} & 1.11027012 & 0.46709738 & -1.01016504 \\ \mathrm{H} & 0.77434015 & -0.07128707 & 4.73093415 \\ \mathrm{H} & 3.18356872 & 0.18338828 & 3.55581147 \\ \mathrm{H} & 4.26443079 & 0.91510513 & -1.94472711 \\ \mathrm{H} & 2.48108234 & 1.09818836 & -3.95330355 \\ \mathrm{~B} & -0.71794739 & 0.81838676 & -7.43833081 \\ \mathrm{C} & -1.72474177 & 0.89833624 & -5.00378663 \\ \mathrm{C} & -3.03200419 & 0.90166088 & -4.42237306 \\ \mathrm{C} & -3.94200777 & 0.98662410 & -5.45080420 \\ \mathrm{C} & -3.19993069 & 1.03854424 & -6.66615153 \\ \mathrm{C} & -3.63211536 & 1.17168644 & -7.98327394 \\ \mathrm{C} & -2.72736233 & 1.28341373 & -9.03599698 \\ \mathrm{C} & -2.94959978 & 1.50146791 & -10.42653139 \\ \mathrm{C} & -1.71468065 & 1.58120270 & -11.02780041 \\ \mathrm{C} & -0.72714036 & 1.40861880 & -10.00697498 \\ \mathrm{~F} & 0.35229661 & 1.66285498 & -7.13968782 \\ \mathrm{~F} & -0.30460089 & -0.50027109 & -7.49006657 \\ \mathrm{~N} & -1.85001020 & 0.98319319 & -6.36022870 \\ \mathrm{~N} & -1.36144117 & 1.23276767 & -8.81135893 \\ \mathrm{H} & -3.24315277 & 0.86213203 & -3.36288629 \\ \mathrm{H} & -5.02100854 & 1.02678134 & -5.37805128 \\ \mathrm{H} & -3.92075299 & 1.59910745 & -10.89394106 \\ \mathrm{H} & -1.50597196 & 1.75597383 & -12.07399812 \\ \mathrm{C} & -0.29530601 & 0.70051449 & -3.00502439 \\ \mathrm{C} & -0.45180556 & 0.84721902 & -4.35184321 \\ \mathrm{H} & -1.16197782 & 0.60566125 & -2.36078964 \\ \mathrm{H} & 0.41526666 & 0.94726805 & -4.99471486 \\ & & & \\ & & & \\ \mathrm{H} & & \end{array}$




\begin{tabular}{|c|c|c|c|}
\hline B & 2.78575349 & 1.45990440 & -14.06000322 \\
\hline $\mathrm{C}$ & 2.78654339 & 1.56835927 & -11.42673046 \\
\hline $\mathrm{C}$ & 3.77127229 & 1.56535355 & -10.38863113 \\
\hline $\mathrm{C}$ & 5.00724731 & 1.61555174 & -10.99079497 \\
\hline $\mathrm{C}$ & 4.78837503 & 1.65280726 & -12.39829906 \\
\hline $\mathrm{C}$ & 5.69480706 & 1.74941582 & -13.45081684 \\
\hline $\mathrm{C}$ & 5.26570412 & 1.85164578 & -14.77169732 \\
\hline $\mathrm{C}$ & 6.00879123 & 2.03302208 & -15.97349763 \\
\hline $\mathrm{C}$ & 5.10030923 & 2.12225399 & -17.00342106 \\
\hline $\mathrm{C}$ & 3.79402209 & 1.99267553 & -16.43602145 \\
\hline $\mathrm{F}$ & 2.38741264 & 0.14948773 & -14.25082597 \\
\hline F & 1.70593764 & 2.33228883 & -14.20269574 \\
\hline $\mathrm{N}$ & 3.42329377 & 1.62412483 & -12.63237529 \\
\hline $\mathrm{N}$ & 3.91655524 & 1.83188486 & -15.08692172 \\
\hline $\mathrm{H}$ & 3.55994180 & 1.54234585 & -9.32871670 \\
\hline $\mathrm{H}$ & 5.97680545 & 1.64010662 & -10.51067910 \\
\hline $\mathrm{H}$ & 7.08704951 & 2.10166679 & -16.03480557 \\
\hline $\mathrm{H}$ & 5.31261369 & 2.27742263 & -18.05200686 \\
\hline $\mathrm{C}$ & 0.69898077 & 1.42360614 & -10.12471499 \\
\hline $\mathrm{C}$ & 1.36028022 & 1.53999270 & -11.31177787 \\
\hline $\mathrm{H}$ & 1.25323514 & 1.34638553 & -9.19632970 \\
\hline $\mathrm{H}$ & 0.80657669 & 1.62826400 & -12.23952360 \\
\hline $\mathrm{H}$ & 3.95475518 & 0.45737479 & 0.83774872 \\
\hline $\mathrm{H}$ & -4.69615262 & 1.21686570 & -8.19077706 \\
\hline $\mathrm{H}$ & 6.75795343 & 1.77229791 & -13.23511742 \\
\hline B & -3.48368051 & -0.37588744 & 6.67373854 \\
\hline $\mathrm{C}$ & -4.44557712 & -1.01903383 & 9.00395211 \\
\hline $\mathrm{C}$ & -5.69569527 & -1.28090241 & 9.60351166 \\
\hline $\mathrm{C}$ & -6.64389273 & -1.23293174 & 8.58874492 \\
\hline $\mathrm{C}$ & -5.94896080 & -0.94721861 & 7.38528623 \\
\hline $\mathrm{C}$ & -6.38150030 & -0.84747756 & 6.06396883 \\
\hline $\mathrm{C}$ & -5.48680391 & -0.66254842 & 5.01443331 \\
\hline $\mathrm{C}$ & -5.71440584 & -0.60502437 & 3.60873102 \\
\hline $\mathrm{C}$ & -4.48769221 & -0.46063121 & 3.00294963 \\
\hline $\mathrm{C}$ & -3.50033073 & -0.42746410 & 4.03871380 \\
\hline $\mathrm{F}$ & -3.16194760 & 0.95407371 & 6.87654956 \\
\hline $\mathrm{F}$ & -2.37075484 & -1.19193177 & 6.81869905 \\
\hline $\mathrm{N}$ & -4.59477279 & -0.81963928 & 7.68515795 \\
\hline $\mathrm{N}$ & -4.12210187 & -0.55150414 & 5.24230415 \\
\hline $\mathrm{H}$ & -5.86236449 & -1.48436613 & 10.65232194 \\
\hline $\mathrm{H}$ & -7.71052250 & -1.39587578 & 8.67163955 \\
\hline $\mathrm{H}$ & -6.68247733 & -0.68319795 & 3.13111528 \\
\hline $\mathrm{H}$ & -4.28206538 & -0.40677295 & 1.94303442 \\
\hline $\mathrm{H}$ & -3.46604147 & -0.96503938 & 9.45981551 \\
\hline $\mathrm{H}$ & -7.43962377 & -0.94513547 & 5.84203630 \\
\hline $\mathrm{C}$ & -1.42525354 & -0.09860934 & 2.74841193 \\
\hline $\mathrm{C}$ & -2.07478787 & -0.32133503 & 3.92430128 \\
\hline B & 2.11715367 & 1.96681428 & -21.51596395 \\
\hline $\mathrm{C}$ & 2.11756300 & 2.40828416 & -24.07969144 \\
\hline $\mathrm{C}$ & 1.19153515 & 2.59549977 & -25.12768847 \\
\hline $\mathrm{C}$ & -0.07170524 & 2.60265729 & -24.54865805 \\
\hline $\mathrm{C}$ & 0.11097830 & 2.42417335 & -23.15305750 \\
\hline $\mathrm{C}$ & -0.79290598 & 2.41089432 & -22.09210935 \\
\hline $\mathrm{C}$ & -0.36689003 & 2.32171780 & -20.77045436 \\
\hline $\mathrm{C}$ & -1.11348809 & 2.36023727 & -19.55709397 \\
\hline $\mathrm{C}$ & -0.21130506 & 2.28774117 & -18.52114394 \\
\hline
\end{tabular}




$\begin{array}{lrrr}\mathrm{C} & 1.09619254 & 2.20263596 & -19.09754039 \\ \mathrm{~F} & 2.49230828 & 0.63647209 & -21.47946442 \\ \mathrm{~F} & 3.20060761 & 2.80324158 & -21.28678611 \\ \mathrm{~N} & 1.47645757 & 2.30598487 & -22.90505781 \\ \mathrm{~N} & 0.98071253 & 2.22467990 & -20.45291437 \\ \mathrm{H} & 1.43789834 & 2.71489860 & -26.17373733 \\ \mathrm{H} & -1.02541284 & 2.73363333 & -25.04303378 \\ \mathrm{H} & -2.19020240 & 2.44817797 & -19.49119154 \\ \mathrm{H} & -0.42644856 & 2.30876125 & -17.46195894 \\ \mathrm{H} & 3.19638991 & 2.34189457 & -24.12362356 \\ \mathrm{H} & -1.85508410 & 2.50039091 & -22.29748587 \\ \mathrm{C} & 2.52078930 & 2.03739827 & -17.09198601 \\ \mathrm{C} & 2.36965302 & 2.13378465 & -18.44205316 \\ \mathrm{H} & 3.23800620 & 2.17671325 & -19.09004295 \\ \mathrm{H} & 1.65137135 & 2.00166882 & -16.44535280 \\ \mathrm{H} & -1.98572782 & 0.02909591 & 1.82914607 \\ \mathrm{H} & -1.51610178 & -0.44671655 & 4.84519263\end{array}$

SCF done: -2954.19301065 Hartree

No imaginary Frequency.

Hexamer 6, optimized $\mathrm{S}_{0}$ state Geometry.

$\begin{array}{lrrr}\text { B } & -10.72217700 & 1.60621500 & 0.14279900 \\ \mathrm{C} & -13.27413200 & 2.24556400 & 0.00796400 \\ \mathrm{C} & -14.04086600 & 3.45123200 & -0.05059400 \\ \mathrm{C} & -13.15520500 & 4.49928700 & -0.15721100 \\ \mathrm{C} & -11.84409100 & 3.94250700 & -0.16751600 \\ \mathrm{C} & -10.60158400 & 4.55886600 & -0.29160800 \\ \mathrm{C} & -9.42536800 & 3.81630300 & -0.35165900 \\ \mathrm{C} & -8.07716400 & 4.23480700 & -0.54484000 \\ \mathrm{C} & -7.29946500 & 3.10022900 & -0.57198100 \\ \mathrm{~F} & -8.16921000 & 1.97822400 & -0.39342800 \\ \mathrm{~F} & -10.63836000 & 1.23354100 & 1.47223800 \\ \mathrm{~N} & -10.84428400 & 0.48574200 & -0.67956900 \\ \mathrm{~N} & -11.95013900 & 2.56436200 & -0.06880800 \\ \mathrm{H} & -9.44889600 & 2.43393100 & -0.26356800 \\ \mathrm{H} & -15.12041900 & 3.50514900 & -0.03439000 \\ \mathrm{H} & -13.38478500 & 5.55374400 & -0.23784100 \\ \mathrm{H} & -7.75477900 & 5.26109000 & -0.66296800 \\ \mathrm{~B} & -6.22997800 & 3.04300700 & -0.71871100 \\ \mathrm{C} & -3.65030600 & -0.89298500 & -0.14216300 \\ \mathrm{C} & -6.24049400 & -1.28761000 & -0.42225400 \\ \mathrm{C} & -7.11766800 & -2.41647100 & -0.47761500 \\ \mathrm{C} & -6.33536900 & -3.54835000 & -0.48270700 \\ \mathrm{C} & -4.97687500 & -3.12124600 & -0.43241300 \\ \mathrm{C} & -3.79626200 & -3.85948900 & -0.45696300 \\ \mathrm{C} & -2.55112400 & -3.23613800 & -0.47397100 \\ \mathrm{C} & -1.24175600 & -3.79019100 & -0.57051100 \\ \mathrm{C} & -0.35575600 & -2.73792400 & -0.58927000 \\ \mathrm{~F} & -1.11913300 & -1.53086900 & -0.50108100 \\ \mathrm{~F} & -3.61128400 & 0.19804700 & -1.01152900 \\ \mathrm{~N} & -3.61002800 & -0.47346200 & 1.17499700 \\ \mathrm{~N} & -4.95187800 & -1.73676800 & -0.39704000 \\ \mathrm{H} & -2.44314300 & -1.85577400 & -0.43548700 \\ \mathrm{H} & -8.19649600 & -2.36609000 & -0.52518700 \\ \mathrm{H} & -6.66267400 & -4.57865800 & -0.53277300 \\ & -1.01590700 & -4.84674900 & -0.63231700\end{array}$




\begin{tabular}{|c|c|c|c|}
\hline $\mathrm{H}$ & 0.72095800 & -2.79010400 & -0.67011400 \\
\hline $\mathrm{C}$ & -7.84343900 & 0.58475300 & -0.36998500 \\
\hline $\mathrm{C}$ & -6.56767900 & 0.10549700 & -0.41939200 \\
\hline $\mathrm{H}$ & -8.68336000 & -0.09881400 & -0.32054400 \\
\hline $\mathrm{H}$ & -5.72857000 & 0.78986200 & -0.47103500 \\
\hline B & 3.65031800 & 0.89295700 & -0.14232900 \\
\hline $\mathrm{C}$ & 1.11912400 & 1.53084600 & -0.50111200 \\
\hline $\mathrm{C}$ & 0.35574300 & 2.73790200 & -0.58923500 \\
\hline $\mathrm{C}$ & 1.24174600 & 3.79016900 & -0.57052900 \\
\hline $\mathrm{C}$ & 2.55111600 & 3.23611600 & -0.47404200 \\
\hline $\mathrm{C}$ & 3.79625300 & 3.85947000 & -0.45706900 \\
\hline $\mathrm{C}$ & 4.97687000 & 3.12123200 & -0.43256900 \\
\hline $\mathrm{C}$ & 6.33536000 & 3.54834300 & -0.48290200 \\
\hline $\mathrm{C}$ & 7.11766600 & 2.41646900 & -0.47782400 \\
\hline $\mathrm{C}$ & 6.24050000 & 1.28760200 & -0.42246000 \\
\hline $\mathrm{F}$ & 3.61008800 & 0.47338600 & 1.17481800 \\
\hline $\mathrm{F}$ & 3.61127100 & -0.19804600 & -1.01173100 \\
\hline $\mathrm{N}$ & 2.44313900 & 1.85575100 & -0.43557600 \\
\hline $\mathrm{N}$ & 4.95188000 & 1.73675300 & -0.39722100 \\
\hline $\mathrm{H}$ & -0.72097500 & 2.79008400 & -0.67001200 \\
\hline $\mathrm{H}$ & 1.01589300 & 4.84672700 & -0.63231700 \\
\hline $\mathrm{H}$ & 6.66265900 & 4.57865300 & -0.53297100 \\
\hline $\mathrm{H}$ & 8.19649300 & 2.36609500 & -0.52541000 \\
\hline $\mathrm{C}$ & -0.65892800 & -0.17601900 & -0.49488200 \\
\hline $\mathrm{C}$ & 0.65891700 & 0.17599800 & -0.49490200 \\
\hline $\mathrm{H}$ & -1.42779100 & 0.58822300 & -0.49889100 \\
\hline $\mathrm{H}$ & 1.42777700 & -0.58824600 & -0.49893300 \\
\hline $\mathrm{H}$ & -10.55101300 & 5.64007400 & -0.36773400 \\
\hline $\mathrm{H}$ & -3.84810900 & -4.94279200 & -0.48925300 \\
\hline $\mathrm{H}$ & 3.84809400 & 4.94277400 & -0.48934400 \\
\hline B & -18.05359800 & -0.13867100 & 0.24917300 \\
\hline C & -20.57035000 & -0.50015400 & 0.79956400 \\
\hline $\mathrm{C}$ & -21.46630700 & -1.56449200 & 1.03469200 \\
\hline C & -20.71277300 & -2.73202600 & 1.02099300 \\
\hline $\mathrm{C}$ & -19.36642200 & -2.35360800 & 0.78221200 \\
\hline $\mathrm{C}$ & -18.18807500 & -3.09626800 & 0.72984700 \\
\hline $\mathrm{C}$ & -16.94585900 & -2.48632900 & 0.58497900 \\
\hline $\mathrm{C}$ & -15.63734600 & -3.05096700 & 0.57811100 \\
\hline $\mathrm{C}$ & -14.74537500 & -2.01059700 & 0.45741400 \\
\hline $\mathrm{C}$ & -15.50645400 & -0.80031800 & 0.38768100 \\
\hline $\mathrm{F}$ & -18.12472400 & 0.22472500 & -1.08366800 \\
\hline $\mathrm{F}$ & -17.94999500 & 0.97464900 & 1.07050900 \\
\hline $\mathrm{N}$ & -19.32155900 & -0.96801600 & 0.64826800 \\
\hline $\mathrm{N}$ & -16.82909800 & -1.10821300 & 0.46772700 \\
\hline $\mathrm{H}$ & -22.53079700 & -1.46882400 & 1.19866300 \\
\hline $\mathrm{H}$ & -21.05959700 & -3.74518300 & 1.17701500 \\
\hline $\mathrm{H}$ & -15.41400400 & -4.10582700 & 0.67173300 \\
\hline $\mathrm{H}$ & -13.66618300 & -2.07076600 & 0.44174400 \\
\hline $\mathrm{H}$ & -20.77065200 & 0.56077400 & 0.73253600 \\
\hline $\mathrm{H}$ & -18.23500200 & -4.17589200 & 0.83298800 \\
\hline $\mathrm{C}$ & -13.73567400 & 0.89226300 & 0.10764500 \\
\hline $\mathrm{C}$ & -15.04257000 & 0.55273800 & 0.28351300 \\
\hline B & 10.72217800 & -1.60619400 & 0.14268200 \\
\hline $\mathrm{C}$ & 13.27414000 & -2.24554900 & 0.00801200 \\
\hline C & 14.04087600 & -3.45121900 & -0.05045100 \\
\hline $\mathrm{C}$ & 13.15522200 & -4.49928000 & -0.15706800 \\
\hline
\end{tabular}




\begin{tabular}{|c|c|c|c|}
\hline $\mathrm{C}$ & 11.84410900 & -3.94249400 & -0.16750800 \\
\hline $\mathrm{C}$ & 10.60161400 & -4.55885900 & -0.29169300 \\
\hline $\mathrm{C}$ & 9.42540100 & -3.81629600 & -0.35182300 \\
\hline $\mathrm{C}$ & 8.07720800 & -4.23480100 & -0.54507700 \\
\hline $\mathrm{C}$ & 7.29950900 & -3.10022500 & -0.57226700 \\
\hline $\mathrm{C}$ & 8.16924000 & -1.97821800 & -0.39365100 \\
\hline $\mathrm{F}$ & 10.63828700 & -1.23348000 & 1.47210700 \\
\hline $\mathrm{F}$ & 10.84433600 & -0.48574600 & -0.67970800 \\
\hline $\mathrm{N}$ & 11.95015100 & -2.56435100 & -0.06882100 \\
\hline $\mathrm{N}$ & 9.44892100 & -2.43392200 & -0.26373600 \\
\hline $\mathrm{H}$ & 15.12042700 & -3.50513700 & -0.03415300 \\
\hline $\mathrm{H}$ & 13.38480300 & -5.55374300 & -0.23761500 \\
\hline $\mathrm{H}$ & 7.75483200 & -5.26108400 & -0.66322500 \\
\hline $\mathrm{H}$ & 6.23003100 & -3.04300700 & -0.71905900 \\
\hline $\mathrm{H}$ & 10.55104900 & -5.64006800 & -0.36780100 \\
\hline $\mathrm{C}$ & 6.56769700 & -0.10550100 & -0.41961300 \\
\hline $\mathrm{C}$ & 7.84346100 & -0.58474800 & -0.37020600 \\
\hline $\mathrm{H}$ & 8.68337800 & 0.09882300 & -0.32075100 \\
\hline $\mathrm{H}$ & 5.72859400 & -0.78987200 & -0.47125200 \\
\hline $\mathrm{H}$ & -12.97700900 & 0.12230200 & 0.02246300 \\
\hline $\mathrm{H}$ & -15.80204000 & 1.32194400 & 0.37009600 \\
\hline B & 18.05356500 & 0.13862000 & 0.24946000 \\
\hline $\mathrm{C}$ & 20.57038300 & 0.50023200 & 0.79940800 \\
\hline $\mathrm{C}$ & 21.46633500 & 1.56459100 & 1.03444900 \\
\hline $\mathrm{C}$ & 20.71276500 & 2.73210200 & 1.02085400 \\
\hline $\mathrm{C}$ & 19.36639900 & 2.35365000 & 0.78221700 \\
\hline $\mathrm{C}$ & 18.18804800 & 3.09630700 & 0.72989100 \\
\hline $\mathrm{C}$ & 16.94583600 & 2.48636000 & 0.58503300 \\
\hline $\mathrm{C}$ & 15.63731500 & 3.05099000 & 0.57816100 \\
\hline $\mathrm{C}$ & 14.74535700 & 2.01061000 & 0.45746600 \\
\hline C & 15.50644600 & 0.80034000 & 0.38773100 \\
\hline $\mathrm{F}$ & 18.12466200 & -0.22521900 & -1.08326200 \\
\hline $\mathrm{F}$ & 17.94992000 & -0.97444200 & 1.07114000 \\
\hline $\mathrm{N}$ & 19.32155800 & 0.96805800 & 0.6482610 \\
\hline $\mathrm{N}$ & 16.82908900 & 1.10825200 & 0.4677560 \\
\hline $\mathrm{H}$ & 22.53084900 & 1.46895100 & $1.1982850 \mathrm{C}$ \\
\hline $\mathrm{H}$ & 21.05957500 & 3.74527000 & 1.17683600 \\
\hline $\mathrm{H}$ & 15.41396700 & 4.10584900 & 0.6717660 \\
\hline $\mathrm{H}$ & 13.66616400 & 2.07076400 & 0.44177600 \\
\hline $\mathrm{H}$ & 20.77071400 & -0.56068800 & 0.73234100 \\
\hline $\mathrm{H}$ & 18.23497600 & 4.17592900 & 0.83304900 \\
\hline $\mathrm{C}$ & 13.73567700 & -0.89224200 & 0.10767400 \\
\hline $\mathrm{C}$ & 15.04256900 & -0.55272200 & 0.28357100 \\
\hline $\mathrm{H}$ & 12.97702100 & -0.12227900 & 0.02245300 \\
\hline $\mathrm{H}$ & 15.80203400 & -1.32192700 & 0.37017300 \\
\hline
\end{tabular}

SCF done: -2954.19301065 Hartree No imaginary Frequency. 


\section{References:}

1. Gaussian 09, Revision D.01, M. J. Frisch, G. W. Trucks, H. B. Schlegel, G. E. Scuseria, M. A. Robb, J. R. Cheeseman, G. Scalmani, V. Barone, B. Mennucci, G. A. Petersson, H. Nakatsuji, M. Caricato, X. Li, H. P. Hratchian, A. F. Izmaylov, J. Bloino, G. Zheng, J. L. Sonnenberg, M. Hada, M. Ehara, K. Toyota, R. Fukuda, J. Hasegawa, M. Ishida, T. Nakajima, Y. Honda, O. Kitao, H. Nakai, T. Vreven, J. A. Montgomery, Jr., J. E. Peralta, F. Ogliaro, M. Bearpark, J. J. Heyd, E. Brothers, K. N. Kudin, V. N. Staroverov, T. Keith, R. Kobayashi, J. Normand, K. Raghavachari, A. Rendell, J. C. Burant, S. S. Iyengar, J. Tomasi, M. Cossi, N. Rega, J. M. Millam, M. Klene, J. E. Knox, J. B. Cross, V. Bakken, C. Adamo, J. Jaramillo, R. Gomperts, R. E. Stratmann, O. Yazyev, A. J. Austin, R. Cammi, C. Pomelli, J. W. Ochterski, R. L. Martin, K. Morokuma, V. G. Zakrzewski, G. A. Voth, P. Salvador, J. J. Dannenberg, S. Dapprich, A. D. Daniels, O. Farkas, J. B. Foresman, J. V. Ortiz, J. Cioslowski, and D. J. Fox, Gaussian, Inc., Wallingford CT, 2013.

2. a) Bhagi, A.; Pandey, S.; Pandey, A.; Pandey, S. Fluorescein prototropism within poly (ethylene glycol) s and their aqueous mixtures. J. Phys. Chem. B 2013, 117, 5230-5240; b) Isak, S.; Eyring, E. J. Phys. Chem. 1992, 96, 1738; c) Benson, R.; Kues, H. Phys. Med. Biol., 1978, 23, 159.

3. a) Casalboni, M.; Matteis, F.; Prosposito, P.; Quatela, A.; Sarcinelli, F. Chem. Phys. Lett. 2003, 373, 372-378; b) Wang, Z.; Liu, Y.; Wang, P.; Jiang, Y.; Ji, M. Nanomedicine (Lond.), 2020, 15, 115-129.

4. a) Benson, R.; Kues, H. Phys. Med. Biol., 1978, 23, 159. b) Lakowicz, J. Principles of Fluorescence Spectroscopy, 3rd ed.; Springer: New York, 2006.

5. SAINT V 6.01 (NT) Software for the CCD Detector System, Bruker Analytical X-ray Systems, Madison, WI 1999.

6. Sheldrick, G. SHELXS-90, Program for the Solution of Crystal Structure, University of Göttingen, Germany, 1990.

7. Sheldrick, G. Acta Cryst. 2015, 71, 3-8.

8. SHELXTL 5.10 (PC/NT-Version), Program library for Structure Solution and Molecular Graphics, Bruker Analytical X-ray Systems, Madison, WI 1998.

9. a) Roper, D.; Ahn, W.; Hoepfner, M. J. Phys. Chem. C 2007, 111, 3636-3641; (b) Zou, Q.; Abbas, M.; Zhao, L.; Li, S.; Shen, G.; Yan, X. J. Am. Chem. Soc. 2017, 139, 1921-1927.

10. Zong, L.; Zhang, H.; Li, Y.; Gong, Y.; Li, D.; Wang, J.; Wang, Z.; Xie, Y.; Han, M.; Peng, Q.; Li, X.; Dong, J.; Qian, J.; Li, Q.; Li, Z. ACS Nano 2018, 12, 9532-9540.

11. Zhao, H.; Wang, B.; Liao, J.; Wang, H.; Tan, G. Tetrahedron Lett. 2013, 54, 6019-6022.

12. Zhou, X.; Yu, C.; Feng, Z.; Yu, Y.; Wang, J.; Hao, E.; Wei, Y.; Mu, X.; Jiao, L. Org. Lett. 2015, 17, $4632-4635$. 